Aus der Abteilung Kardiologie und Pneumologie

(Prof. Dr. med. G. Hasenfuß)

im Zentrum Innere Medizin

der Medizinischen Fakultät der Universität Göttingen

\title{
Einfluss einer diabetischen Stoffwechsellage auf die diastolische Funktion des linken Ventrikels
}

\author{
I NA UG URAL - DISSERTATION \\ zur Erlangung des Doktorgrades \\ der Medizinischen Fakultät \\ der Georg-August-Universität zu Göttingen
}

vorgelegt von

Lisa Christiane Schönbrunn

aus

Frankfurt am Main

Göttingen 2011 
Dekan: Prof. Dr. med. C. Frömmel

I. Berichterstatter: Prof. Dr. med. G. Hasenfuß

II. Berichterstatter/in: Prof. Dr. med. D. Raddatz

Tag der mündlichen Prüfung: 10. August 2011 


\section{Inhaltsverzeichnis}

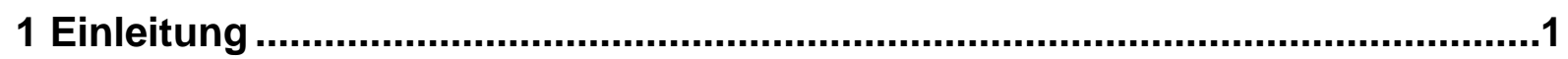

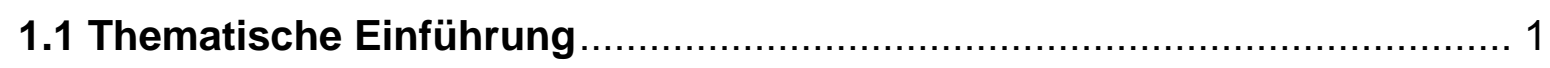

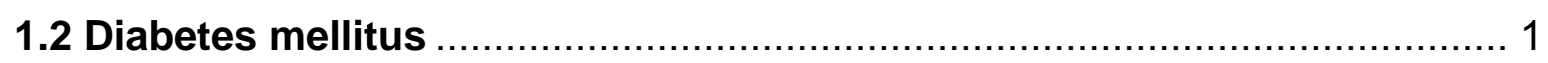

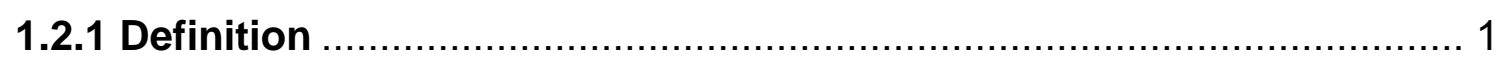



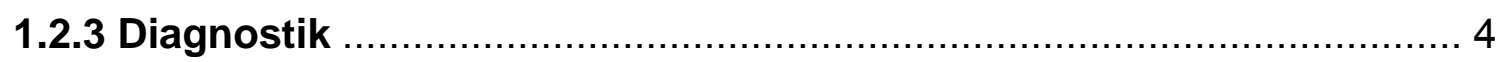

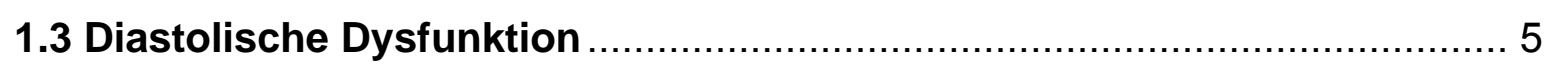

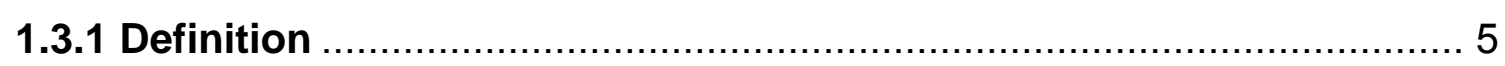

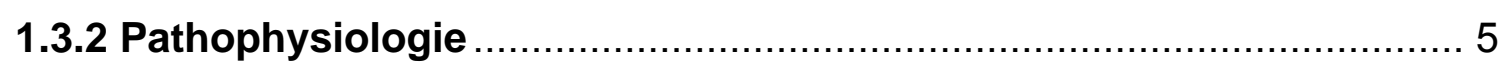

1.3.3 Zelluläre Mechanismen .................................................................. 7

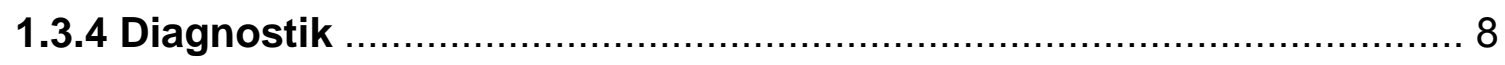

1.3.5 Epidemiologie und prognostische Bedeutung .............................. 10

1.4 Diabetes und diastolische Dysfunktion .............................................. 11

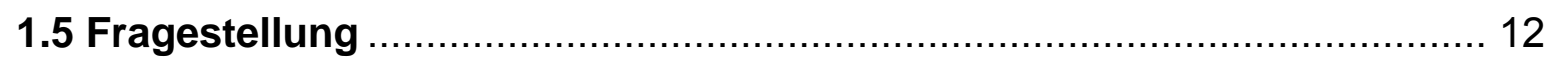

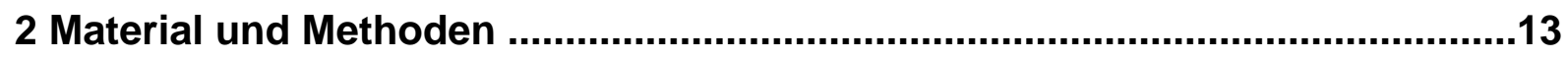



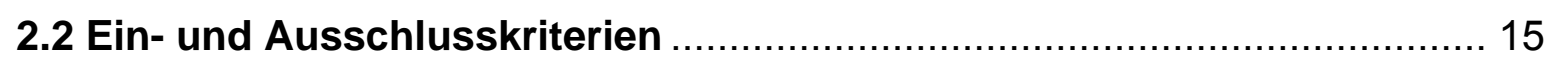

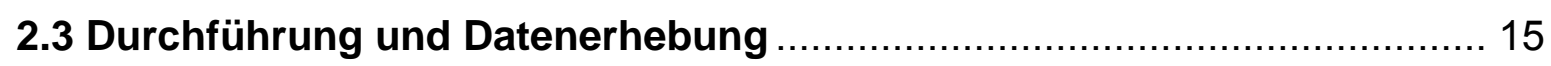

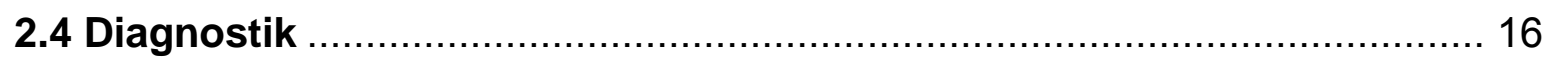

2.4.1 Diabetes mellitus und oraler Glucosetoleranztest............................ 16

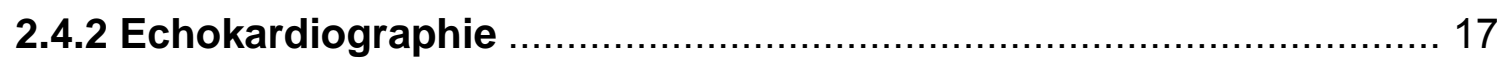

2.4.2.1 Füllungsvolumina und Ejektionsfraktion ........................................ 17

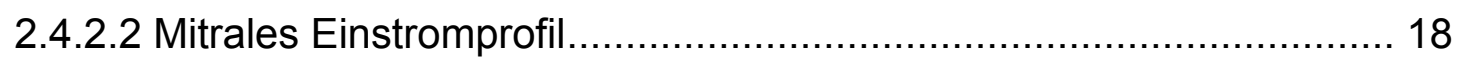

2.4.2.3 Isovolumetrische Relaxationszeit ................................................... 18

2.4.2.4 Mitralanulusgeschwindigkeiten ................................................ 19



2.4.2.6 Beurteilung und Klassifikation der diastolischen Dysfunktion............ 19

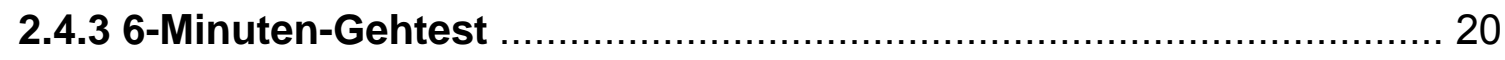

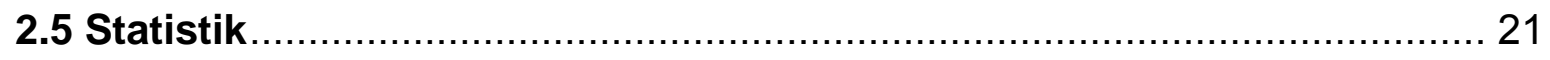

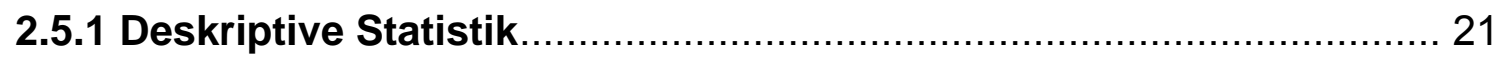

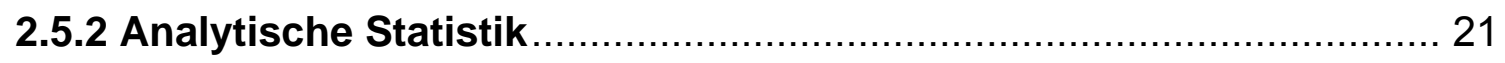

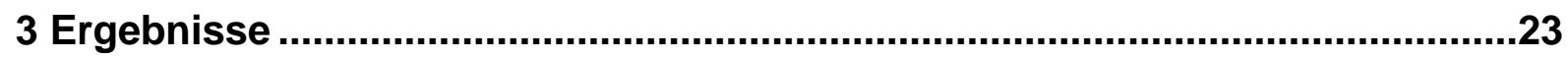




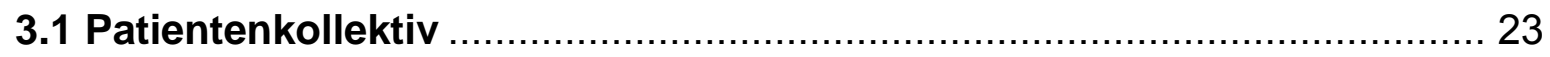

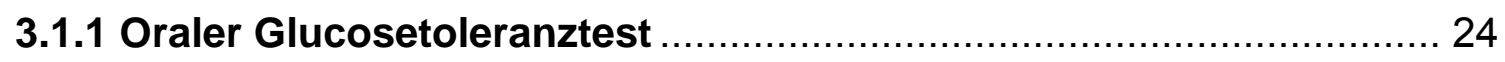

3.1.2 Allgemeine Charakteristika der einzelnen Gruppen ........................ 25

3.1.3 Komorbiditäten und Risikofaktoren .............................................. 27

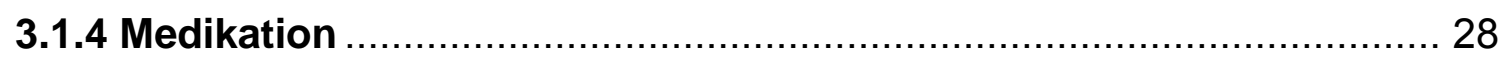

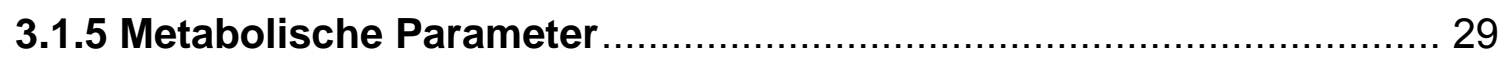

3.1.5.1 Parameter des Glucosestoffwechsels .......................................... 29

3.1.5.2 Weitere metabolische Parameter ................................................. 30

3.2 Diastolische Dysfunktion im Kollektiv ................................................ 31

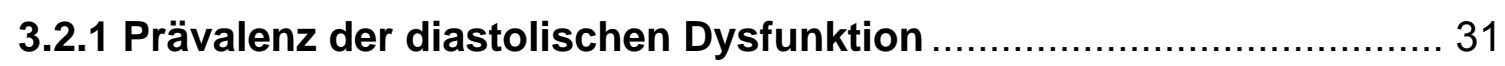

3.2.2 Echokardiographische Parameter ................................................ 34

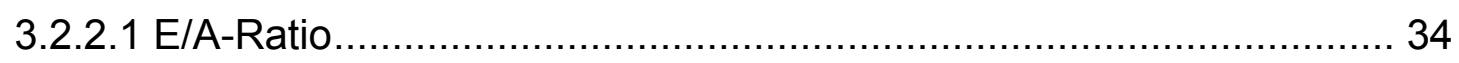

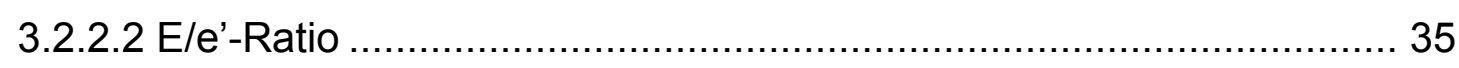

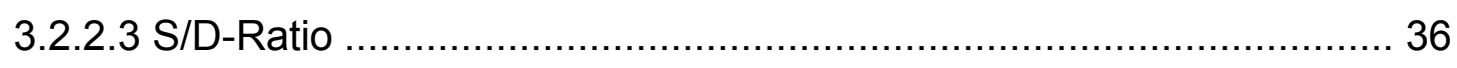

3.2.2.4 Weitere echokardiographische Parameter der diastolischen Funktion 36

3.2.3 Korrelation von Glucosehomöostase und echokardiographischen

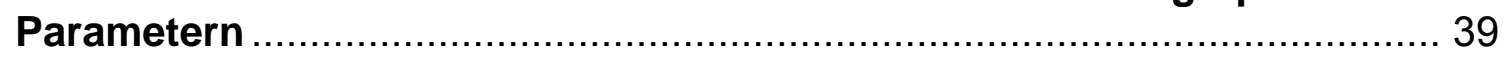

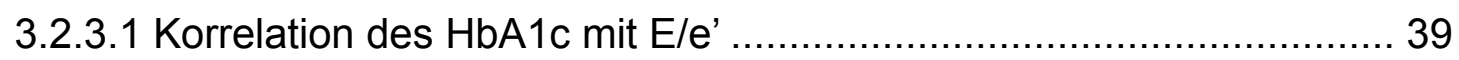

3.2.3.2 Korrelation von Stoffwechselparametern mit LVMI und LAD ............ 39

3.2.4 Der 6-Minuten-Gehtest : Glucosehomöostase, diastolische Dysfunktion und körperliche Leistungsfähigkeit................................... 40

3.2.5 Geschlechtsspezifische Unterschiede der diastolischen Funktion .. 41

3.3 Echokardiographische Parameter der systolischen Ventrikelfunktion ... 41

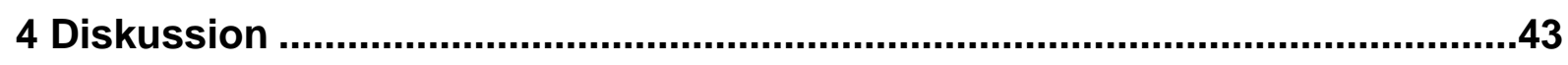

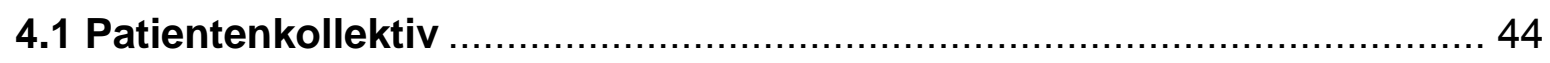

4.2 Übergewicht und Diabetes................................................................... 45

4.3 Glucosehomöostase und Risikofaktoren der diastolischen Dysfunktion 46 4.4 Prävalenz der diastolischen Dysfunktion bei Nicht-Diabetikern ............. 48

4.5 Diastolische Dysfunktion bei Diabetikern .............................................. 49

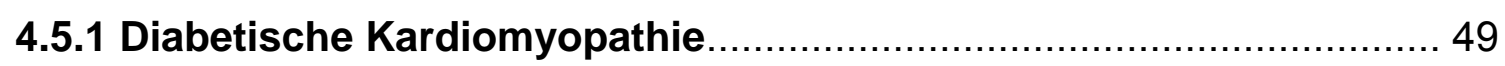

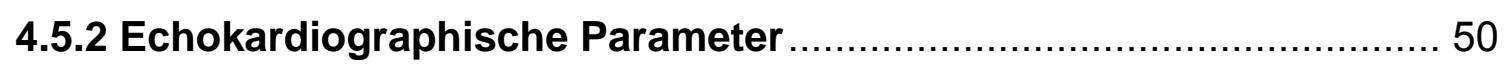

4.5.3 Reversibilität der Diastolischen Dysfunktion beim Diabetiker ......... 52

4.5.4 Diastolische Funktion und Dauer der Diabeteserkrankung .............. 52

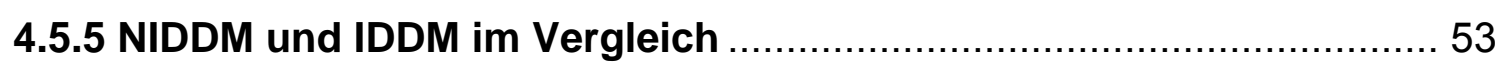

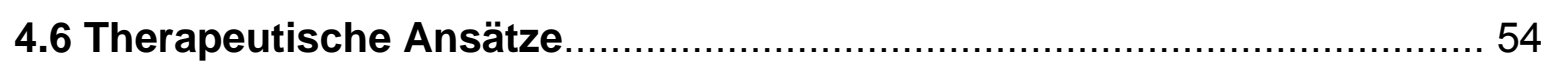


4.7 Geschlechtsspezifische Unterschiede ......................................... 55

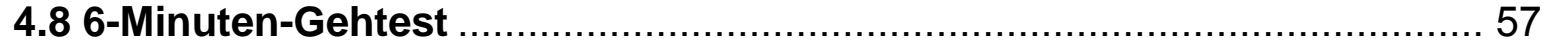

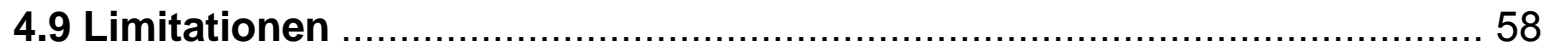

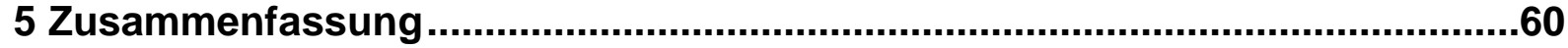

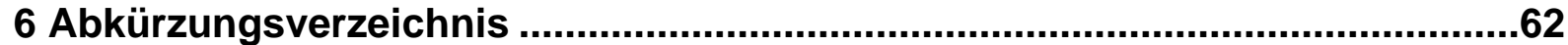

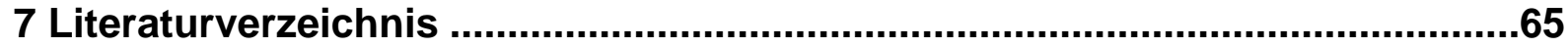




\section{Einleitung}

\subsection{Thematische Einführung}

Diabetes mellitus ist ein unabhängiger Risikofaktor für kardiovaskuläre Erkrankungen und Herzinsuffizienz (Kannel et al. 1974; Wilson et al. 1998). Die häufigste Todesursache bei Diabetikern sind kardiovaskuläre Erkrankungen, insbesondere die koronare Herzerkrankung (Roglic et al. 2005). Weltweit steigt die Häufigkeit des Diabetes mellitus stetig an. Schätzungen der WHO zufolge wird sich die Zahl der Diabetiker von 171 Millionen im Jahr 2000 auf 366 Millionen im Jahr 2030 erhöhen (Wild et al. 2004).

Bei Diabetes mellitus tritt jedoch, unabhängig von koronarer Herzerkrankung und Bluthochdruck, eine zusätzliche Schädigung des Herzens auf, so dass die Existenz einer eigenständigen diabetischen Kardiomyopathie diskutiert wird (Regan et al. 1977; Fang et al. 2004). Bei dieser führt vermutlich die Akkumulation von glycosylierten Endprodukten, sogenannten AGEs (Advanced glycosylation end products), im Myokard zu einer vermehrten Vernetzung von Kollagen und zur myokardialen Fibrose (Candido et al. 2003). Die diastolische Dysfunktion, gekennzeichnet durch gestörte Relaxation und verminderte Compliance des Herzens während der Diastole, wird insbesondere als frühes Zeichen einer beginnenden diabetischen Kardiomyopathie angesehen (Raev 1994). Sie ist somit von besonderer Bedeutung für die Früherkennung und Behandlung des diabetisch geschädigten Herzens.

Im Folgenden sollen genauere Zusammenhänge zwischen der Erkrankung eines Diabetes mellitus und Entstehung und Verlauf einer diastolischen Dysfunktion erörtert werden.

\subsection{Diabetes mellitus}

\subsubsection{Definition}

„Eine wunderbare Krankheit ist der Diabetes, und nicht sehr häufig bei den Menschen. Fleisch und Bein schmilzt in Urin zusammen. [...] Die Kranken haben einen unauslöschlichen Durst und trinken und harnen sehr viel, indessen übersteigt 
die Quantität des gelassenen Urins doch noch die des Getränkes. [...] Und daher auch hat, wie ich glaube, die Krankheit den Namen Diabetes erhalten, als wenn sie ein Weinheber wäre, weil nämlich die Flüssigkeit nicht im Körper bleibt, sondern den Menschen wie eine Röhre benutzt, durch welche sie abfließen kann." (Aretaios, aus dem Griechischen von Mann 1858, S. 85-86)

Diese, wohl erste bekannte, detaillierte Beschreibung des Diabetes in der Medizingeschichte stammt von dem griechischen Arzt Aretaios von Kappadokien (ca. 80-130 n. Chr.). Schon um das Jahr 100 n. Chr. erkannte Aretaios die typische Symptomatik dieser Erkrankung, die sich unter anderem durch Polyurie und Polydipsie äußert. Aretaios bezeichnete die Krankheit als ,Diabetes' in Anlehnung an das griechische Wort , $\delta 1 \alpha \beta \alpha$ lv $\omega$ ' (,diabaino'), was soviel wie ,ich gehe' oder ,ich fließe hindurch' bedeutet (Henschen 1969). Der Zusatz ,mellitus', lateinisch für ,honigsüß', wurde erst später von dem Briten William Cullen eingeführt, der 1769 seine Beobachtungen zum süßen Geschmack des Urins bei Diabetikern veröffentlichte (Sanders 2002).

Die WHO definiert Diabetes als eine metabolische Stoffwechselstörung multifaktorieller Ätiologie, charakterisiert durch eine chronische Hyperglykämie. Ursache der Hyperglykämie kann zum einen eine gestörte Insulinsekretion, zum anderen eine gestörte Insulinwirkung oder eine Kombination beider Mechanismen sein. Wie schon Aretaios 100 n. Chr., so führt auch die WHO Polydipsie und Polyurie als Hauptsymptome der Erkrankung an. Ergänzend werden außerdem Gewichtsverlust und verschwommenes Sehen genannt (World Health Organization 1999).

Die chronische Hyperglykämie führt langfristig zu den typischen Folgeerkrankungen wie peripherer und autonomer Neuropathie, sowie zu einer diabetischen Mikroangiopathie mit Retinopathie und Nephropathie. Außerdem kommt es zu einer diabetischen Makroangiopathie, so dass insbesondere das Gehirn, die peripheren Arterien und, was im Folgenden noch genauer betrachtet werden soll, das Herz geschädigt werden (Stratton et al. 2000; Smith et al. 2002).

Akut lebensbedrohliche Komplikationen des Diabetes mellitus sind eine Hyperglykämie mit Ketoazidose und die nicht ketotische Hyperosmolarität (Expert Committee on the Diagnosis and Classification of Diabetes Mellitus 2003). 


\subsubsection{Typen des Diabetes}

Der Großteil der Diabetesfälle lässt sich ätiopathologisch in zwei große Gruppen einteilen: Diabetes mellitus Typ 1 und Typ 2. Dem Diabetes mellitus Typ 1A liegt eine zellvermittelte autoimmune Zerstörung der $\beta$-Zellen des Pankreas zugrunde (Atkinson und MacLaren 1994). Der Diabetes mellitus Typ 1B ist eine idiopathische Form, bei der keine Marker eines Autoimmunprozesses nachweisbar sind. Diese Form ist in hohem Maße erblich, in Deutschland jedoch selten. Insgesamt findet sich beim Diabetes mellitus Typ 1 ein Insulinmangel, der in seinem Ausprägungsgrad von einer gestörten Glucosetoleranz bis hin zur ketoazidotischen Stoffwechselentgleisung mit absoluter Insulinbedürftigkeit reichen kann. Der Typ-1-Diabetes findet sich meist bei jüngeren Patienten, kann sich jedoch in seltenen Fällen auch spät manifestieren. Diese späte Form wird als ,Late Autoimmune Diabetes in Adults' (LADA) bezeichnet (Kerner und Brückel 2008).

Die weitaus häufigere Form des Diabetes ist der Typ 2. In den westlichen Ländern beträgt ihr Anteil ca. 90\% (American Diabetes Association 1998). Im Zuge dieser Arbeit soll ausschließlich diese Form des Diabetes beleuchtet werden. Im Gegensatz zum Diabetes mellitus Typ 1 tritt der Typ 2 meist im erwachsenen Alter auf. Nur selten kommt es zu schweren Stoffwechselentgleisungen, häufig jedoch, infolge der chronischen Hyperglykämie, zu schwerer Mikro- und Makroangiopathie (Fujimoto et al. 1987; Meigs et al. 2002). Oft bleibt die Krankheit über viele Jahre hinweg asymptomatisch, sodass die Hyperglykämie langsam und unbemerkt in den einzelnen Geweben funktionelle Veränderungen hervorruft (Zimmet 1992).

Beim Typ-2-Diabetes spielt eine genetische Disposition eine große Rolle; eine HLAAssoziation besteht jedoch nicht (Barnett et al. 1981; Medici et al. 1999). Barnett et al. zeigten 1981, dass in bis zu 90\% der Fälle eineiige Zwillinge beide im Laufe ihres Lebens an Diabetes mellitus Typ II erkranken.

Ursächlich liegt dem Typ-2-Diabetes eine Kombination aus inadäquater Insulinsekretion und einer verminderten Insulinantwort des Gewebes, einer so genannten Insulinresistenz, zugrunde (Olefsky et al. 1982). Individuell kann pathogenetisch die eine oder die andere Komponente überwiegen.

Risikofaktoren für Diabetes mellitus Typ 2 sind Übergewicht (Schienkiewitz et al. 2006), ungesunde Ernährung, mangelnde körperliche Aktivität, Bluthochdruck und höheres Lebensalter (Lindström und Tuomilehto 2003). 
Das bei den Patienten meist vorhandene Übergewicht bzw. die Adipositas selbst erzeugen eine gewisse Insulinresistenz, was die Krankheitsentstehung begünstigt, bzw. den Krankheitsverlauf verschlimmert (Bogardus et al. 1985).

Auf andere seltenere Diabetesformen, wie z.B. den Typ MODY (Maturity onset diabetes of the young) oder den Gestationsdiabetes soll hier nicht näher eingegangen werden.

\subsubsection{Diagnostik}

Laut den aktuellen Leitlinien der Deutschen Diabetes Gesellschaft (Kerner und Brückel 2009), welche sich an Empfehlungen der WHO, der American Diabetes Association und der International Diabetes Federation orientieren, ist ein Diabetes bei Erfüllung einer der folgenden Kriterien diagnostiziert:

1. bei klassischen Symptomen des Diabetes wie Polyurie, Polydipsie und unklarem Gewichtsverlust sowie einer Gelegenheitsglucose im venösen Plasma oder kapillären Vollblut von $\geq 200 \mathrm{mg} / \mathrm{dl}(11,1 \mathrm{mmol} / \mathrm{l})$

2. bei wiederholter Bestätigung einer Gelegenheitsblutglucose von $\geq 200 \mathrm{mg} / \mathrm{dl}$ $(11,1 \mathrm{mmol} / \mathrm{l})$ im venösen Plasma oder kapillären Vollblut

3. bei zweimaliger Nüchternblutglucose (nach 8stündigem Fasten) von $\geq 126 \mathrm{mg} / \mathrm{dl}$ im venösen Plasma oder $\geq 110 \mathrm{mg} / \mathrm{dl}$ im kapillären Vollblut

4. bei einem oGTT-2h-Wert im venösen Plasma oder kapillären Vollblut $\geq$ $200 \mathrm{mg} / \mathrm{dl}(11,1 \mathrm{mmol} / \mathrm{l})$.

Von einer abnormen Nüchternglucose, im Englischen ,Impaired Fasting Glucose' (IFG), spricht man bei einer Nüchternglucose von $100 \mathrm{mg} / \mathrm{dl}$ bis $126 \mathrm{mg} / \mathrm{dl}$ im venösen Plasma oder ab 90mg/dl im kapillären Vollblut. Die gestörte Glucosetoleranz, im Englischen ,Impaired Glucose Tolerance' (IGT), ist definiert als ein 2h-oGTT-Wert von $140 \mathrm{mg} / \mathrm{dl}$ bis $200 \mathrm{mg} / \mathrm{dl}$ (Kerner und Brückel 2008).

Der HbA1c-Wert gilt als Gold-Standard für die Verlaufskontrolle beim Diabetiker. Er ist ein guter Marker für die durchschnittlichen Plasmaglucosespiegel der letzten zwei bis drei Monate. Die Messung kann zu jeder beliebigen Tageszeit erfolgen und der Patient muss nicht nüchtern sein. Ziel der Diabetes-Therapie sind HbA1c-Werte unter $6,5 \%$, besser aber unter $6 \%$, um das Risiko für die oben erwähnten Folgeschäden möglichst gering zu halten. Der WHO zufolge eignet sich der HbA1c-Wert nicht zur 
Diabetes-Diagnostik (World Health Organization 2006). Unlängst forderte die American Diabetes Association jedoch, HbA1c-Werte ab 6,5\% zur Diagnose eines Diabetes mellitus zuzulassen (International Expert Committee 2009). Sie begründete dies unter anderem mit den Fortschritten bei der Standardisierung des HbA1cWertes, einer geringeren Anfälligkeit gegenüber tagesabhängigen Schwankungen verglichen mit Blutzucker-Werten und der einfachen Durchführbarkeit einer HbA1cWert-Bestimmung. Diese Empfehlung der ADA ist jedoch Gegenstand aktueller Diskussion (Lippi et al. 2010) und hat bislang noch keinen Einzug in aktuelle Leitlinien der Deutschen Diabetes Gesellschaft gefunden (Kerner und Brückel 2009).

\subsection{Diastolische Dysfunktion}

\subsubsection{Definition}

Die diastolische Dysfunktion ist eine mechanische Funktionsstörung des Herzens während der Diastole. Die normalen diastolischen Prozesse des Herzens wie Erschlaffung und Ausdehnung des Ventrikels sind, primär bedingt durch verminderte Relaxation und erhöhte Steifigkeit des Herzens, verkürzt, bzw. unvollständig (Dickstein et al. 2008).

Sofern keine zusätzliche systolische Funktionsstörung besteht, ist bei der diastolischen Dysfunktion die Ejektionsfraktion des Ventrikels normal oder nur leicht vermindert (Zile und Brutsaert 2002a; Hess 2003). Finden sich Symptome einer Herzinsuffizienz bei einer dominierenden oder isolierten diastolischen Funktionsstörung, so spricht man vom klinischen Syndrom der diastolischen Herzinsuffizienz (Vasan und Levy 2000). Die diastolische Dysfunktion kann also isoliert, und somit asymptomatisch, oder aber im Rahmen einer diastolischen Herzinsuffizienz auftreten, bzw. sich zu dieser entwickeln (Zile und Brutsaert 2002a).

\subsubsection{Pathophysiologie}

Durch die verminderte aktive Relaxation oder erhöhte passive Steifigkeit (verringerte Compliance) des Herzens kommt es zu einer Zunahme des diastolischen Füllungsdruckes und einer verminderten Kammerfüllung. Somit ist der linke Ventrikel bei diastolischer Dysfunktion nicht mehr in der Lage, ein ausreichendes Schlagvolumen zu gewährleisten. 
Nach Zile (1989) lässt sich die Diastole in vier verschiedene Phasen unterteilen:

1) Isovolumetrische Relaxation: Diese Phase erstreckt sich vom Schluss der Aortenklappe bis zur Öffnung der Mitralklappe. Der linksventrikuläre Druck fällt stetig, während das linksventrikuläre Volumen konstant bleibt. In dieser Phase spielt die aktive Relaxation eine Hauptrolle.

2) Frühe Füllungsphase: Sie beginnt, wenn der linksventrikuläre Druck unter den Druck des linken Vorhofes fällt. Durch aktive Relaxation des Ventrikels wird ein negativer Druck, eine Art Sogeffekt, erzeugt. Dieser hängt von sympathischer Stimulation ab, sodass auch bei Tachykardie eine adäquate linksventrikuläre Füllung erreicht wird. Um eine ausreichende Relaxation des Ventrikels zu gewährleisten, sind in dieser Phase intakte visko-elastische Anteile, gleichzusetzen mit der Compliance, von besonderer Wichtigkeit. Das Blut strömt aus dem Vorhof in den linken Ventrikel entlang der Mitralklappe zunächst mit zunehmender Geschwindigkeit bis zu $70 \mathrm{~cm} / \mathrm{s}$, während die kontraktile Anspannung des Ventrikels und der Ventrikeldruck zunächst noch abnehmen. Anschließend kommt es wieder zu einem langsamen Druckanstieg im Ventrikel. Die frühe Füllungsphase trägt zu etwa $75 \%$ zur Ventrikelfüllung bei.

3) Diastase: Die Drücke des linken Atriums und des linken Ventrikels sind fast gleich. Die linksventrikuläre Füllung wird hauptsächlich durch den Blutfluss von den Pulmonalvenen aufrechterhalten. Die einfließende Blutmenge hängt vom linksventrikulären Druck und der linksventrikulären Compliance ab.

4) Späte (aktive) Füllungsphase: Das linke Atrium kontrahiert. Die Phase endet mit dem Schluss der Mitralklappe. Sie wird hauptsächlich beeinflusst durch die linksventrikuläre Compliance, hängt aber auch vom Widerstand des Perikards, der atrialen Kontraktionskraft und der atrioventrikulären Synchronität ab.

Es wird klar, dass Beeinträchtigungen, welche einzelne Komponenten der diastolischen Phasen, wie frühe aktive Relaxation oder spätdiastolische Compliance, betreffen, Störungen in den jeweiligen Phasen zur Folge haben müssen. Anders gesagt ist also eine diastolische Dysfunktion jede Störung, die eine verschlechterte 
Relaxation, eine verschlechterte Füllung des Ventrikels oder Verlust der atrialen Kontraktion zur Folge hat (Ommen und Nishimura 2003).

Eine diastolische Dysfunktion findet sich häufig als Folge einer linksventrikulären Hypertrophie, zum Beispiel im Rahmen einer Aortenstenose oder einer hypertrophen obstruktiven Kardiomyopathie (Gwathmey et al. 1991). Meist kommt eine diastolische Dysfunktion in diesem Zusammenhang jedoch bei älteren Patienten mit einer langjährig bestehenden Hypertonie vor. Pathophysiologisch wird hier durch eine Fibroseinduktion die Steifigkeit des Ventrikels zusätzlich verstärkt (Brilla et al. 1992). Insgesamt führt bei diesen Krankheitsbildern eine vermehrte Steifigkeit des Herzens (Restriktion) zur diastolischen Funktionsstörung. Hinzuzufügen sind an dieser Stelle außerdem die restriktiven Kardiomyopathien, welche durch infiltrative (Amyloidose) und nicht infiltrative Mechanismen, sowie Speicherkrankheiten hervorgerufen werden können. Weitere Ursachen einer diastolischen Dysfunktion können zum einen, wie die Pericarditis constrictiva, mit einer Füllungsbehinderung durch das Perikard (Konstriktion) einhergehen oder aber die aktive Relaxation betreffen, wie zum Beispiel im Rahmen ischämischer Herzerkrankungen.

Eine typische Komplikation der diastolischen Dysfunktion ist das Vorhofflimmern, da durch den Anstieg des diastolischen Füllungsdruckes der Vorhof dilatiert. Es kann außerdem zu einer sekundären pulmonalen Hypertonie kommen (Tsang et al. 2002; Lam et al. 2009).

\subsubsection{Zelluläre Mechanismen}

Ätiopathologisch liegen einer diastolischen Funktionsstörung verschiedene Mechanismen zu Grunde. Diese können zum einen den Kardiomyozyten selbst betreffen oder aber extramyokardialer Genese sein. Innerhalb des Kardiomyozyten spielen unter anderem Störungen der Calciumhomöostase eine Rolle. Abnorme Veränderungen an Sarkolemm, sarkoplasmatischem Retikulum und Proteinen des Calciumstoffwechels führen zu einer pathologischen Erhöhung der diastolischen Calciumkonzentration im Zytosol, einem verlängerten Calcium-Transienten und einem verspäteten und verlangsamten diastolischen Abfall der CalciumKonzentration (Apstein und Morgan 1994).

Bei kontinuierlicher aktiver Relaxation wird ATP-abhängig intrazelluläres Calcium in das sarkoplasmatische Retikulum aufgenommen. Da bei diesem Prozess Energie 
verbraucht wird, resultiert eine diastolische Dysfunktion, wenn die Konzentrationen von ADP oder Pi oder das Verhältnis von ADP zu ATP steigen.

Außerdem führen Veränderungen an zytoskelettalen Proteinen des Kardiomyozyten, wie den Microtubuli, Intermediärfilamenten, Mikrofilamenten und endosarkomeren Proteinen zu diastolischer Funktionsstörung (Zile und Brutsaert 2002b).

Eine extramyokardiale pathologische Veränderung, die zu diastolischer Dysfunktion führen kann, betrifft die Extrazellulärmatrix. Die bedeutendste Rolle spielt hier wahrscheinlich das fibrilläre Kollagen Typ 1 und 3, welches neben Proteoglykanen und Membranproteinen einen wichtigen Bestandteil der Extrazellulärmatrix darstellt. Ist diese verändert, so ist die Umgebung der Kardiomyozyten und damit ihre korrekte strukturelle Anordnung und Verbindung untereinander gestört. Es kommt so zur Beeinträchtigung von Kontraktion und Relaxation (Weber 1989).

Akute und chronische neurohumorale und kardiale endotheliale Aktivierung oder Hemmung kann die diastolische Funktion verändern. Eine chronische Aktivierung des RAAS-Systems führt beispielsweise zu einer Vermehrung des fibrillären Kollagens der Extrazellulärmatrix (Brutsaert et al. 1998).

\subsubsection{Diagnostik}

Prinzipiell kann die diastolische Funktion des linken Ventrikels mit verschiedenen Methoden beurteilt werden. Nicht invasive Techniken schließen beispielsweise die Doppler-Echokardiographie, den Gewebedoppler, die Radionuklid-Angiographie und die Magnetresonanztomographie ein. Das linksventrikuläre Konduktanzkathetersystem ist eine invasive Methode, welche eine direkte Messung des linksventrikulären enddiastolischen Druckes ermöglicht (Gutierrez und Blanchard 2004).

Die bedeutendste nicht invasive Methode zur Diagnostik der diastolischen Dysfunktion ist die transthorakale Echokardiographie. Wie auch in der hier zu diskutierenden Studie wird diese sehr häufig zur Beurteilung diastolischer Funktion eingesetzt, da sie gut verfügbar, ohne größeren Aufwand durchführbar und mit geringen Kosten verbunden ist. Ist im M-Mode oder der 2-D-Echokardiographie eine Hypertrophie des linken Ventrikels erkennbar, so ist dies wegweisend für eine eventuell bestehende diastolische Dysfunktion. Weitere Zeichen, die auf eine diastolische Funktionsstörung hinweisen, sind: Dilatation des linken Vorhofs, ein normal weiter oder in Folge der Hypertrophie verkleinert wirkender Ventrikel, eine 
normale oder geringgradig eingeschränkte linksventrikuläre Funktion, sowie eventuell ein hämodynamisch nicht relevanter Perikarderguss (Zile et al. 2001).

Insgesamt haben aber Parameter der Strömungsdynamik eine größere Aussagekraft als morphologische Befunde dieser Art.

Eine Störung der linksventrikulären Relaxation ist echokardiographisch durch einen reduzierten frühdiastolischen Einstrom in den Ventrikel detektierbar. Das veränderte diastolische Flussprofil kann anhand von pw-Doppler über der Mitralklappe abgeleitet werden. Normalerweise fließen während der frühen Diastole ca. $75 \%$ des diastolischen Blutflusses durch die Mitralklappe. Daher übersteigt dann die E-Welle, als Ausdruck des frühdiastolischen Einstroms, die spätdiastolische A-Welle, welche die atriale Kontraktion reflektiert. Das E/A-Verhältnis ist also beim gesunden Erwachsenen unter 65 Jahren immer $>1$ (Erbel et al. 2002). Die European Society of Cardiology (ESC) unterscheidet in ihren Leitlinien drei Typen der diastolischen Füllungsstörung (Dickstein et al. 2008): Im frühen Stadium der diastolischen Dysfunktion findet sich als Ausdruck einer verminderten frühdiastolischen Relaxation ein E/A-Verhältnis von <1. Die Füllung des Ventrikels ist dann insbesondere von der späten atrialen Kontraktion abhängig. Zusätzlich wird die A-Welle durch eine unvollständige Entleerung des Vorhofs und eine daraus resultierende erhöhte Vorlast verstärkt (Ommen und Nishimura 2003). Dieses Stadium kommt insbesondere bei älteren Patienten oder Hypertonikern vor. Meist sind die linksventrikulären Drücke noch normal oder leicht erhöht. In einem Zwischenstadium der diastolischen Dysfunktion normalisiert sich das E/A-Verhältnis wieder, ebenso die Dezelerationszeit (Dauer des Geschwindigkeitsabfalls) der E-Welle (EDCT). Dieses Phänomen bezeichnet man als Pseudonormalisation. Durch ein Valsalva-Manöver kann die Pseudonormalisation demaskiert werden. Hierbei wird die E/A-Ratio wieder $<1$. Überwiegt im späten Stadium die restriktive Komponente der diastolischen Funktionsstörung, so kommt es zu einer verkürzten E-Wellen-Dezelerationszeit, einer erhöhten Maximalgeschwindigkeit der E-Welle sowie einer überhöhten E/A-Ratio. Häufig sind dann erhöhte linksatriale Drücke, verminderte linksventrikuläre Compliance, Volumenüberbelastung oder eine Mitralinsuffizienz zu finden (Appleton et al. 1988).

Die isovolumetrische Relaxationszeit (IVRT) kann mittels gepulster DopplerRegistrierung ( $p w$-Doppler) bestimmt werden. Ist sie verlängert, so deutet dies auf 
eine verlangsamte Relaxation des linken Ventrikels in der frühen Diastole hin (Myreng und Smiseth 1990).

Ein weiterer Parameter, der zur Beurteilung der diastolischen Funktion des Ventrikels beiträgt, ist das Pulmonalvenenflussprofil im Doppler-Spektrum. Der Pulmonalvenenfluss reflektiert die zyklischen Druckschwankungen des linken Vorhofs (Cohen et al. 1998). Normalerweise ist hier eine größere systolische, eine diastolische und eine kleinere spätdiastolische Welle, entsprechend der Vorhofkontraktion, zu erkennen. Findet sich eine Dominanz der diastolischen Welle, so spricht dies für eine diastolische Dysfunktion. Dieser Parameter ist jedoch, wie auch die IVRT, die E/A-Ratio und die Dezelerationszeit vorlastabhängig (Hess 2003). Eine vorlastunabhängige Methode ist die Bestimmung der Exkursion des Mitralringes mittels Gewebedopplerechokardiographie (TDI). Hier werden Mitralringspitzengeschwindigkeiten (e', a') bestimmt. Der Gewebedoppler bietet zum einen die Möglichkeit, ein pseudonormales Flussprofil zu erkennen, da bei Pseudonormalisierung e'<a' ist. Die E/e'-Ratio korreliert zudem mit dem linksventrikulären enddiastolischen Druck, so dass bei ihrer Berechnung auch bei Vorhofflimmern eine Aussage bezüglich der Druckverhältnisse des linken Vorhofs getroffen werden kann (Ommen et al. 2000).

Zusammenfassend lässt sich die diastolische Funktion also in vier Schweregrade unterteilen: 1) normale diastolische Funktion, 2) leichte diastolische Dysfunktion (Relaxationsstörung), 3) mäßige diastolische Dysfunktion (Pseudonormalisation) und 4) schwere diastolische Dysfunktion (Restriktion) (Nishimura und Tajik 1997).

\subsubsection{Epidemiologie und prognostische Bedeutung}

Allgemein gültige Angaben zur Prävalenz der diastolischen Dysfunktion sind bisher nicht bekannt. Fischer et al. fanden 2003 eine diastolische Funktionsstörung bei $11,1 \%$ der rund 1700 Probanden zwischen 25 und 75 Jahren. Bei einer großen, bevölkerungs-basierten Studie von Redfield et al. (2003) hatten 20,3\% der Probanden eine milde, 6,6\% eine moderate und 0,7\% eine schwere diastolische Dysfunktion. Insgesamt nimmt die Prävalenz der diastolischen Dysfunktion mit dem Alter zu (Kitzman et al. 1991). Unabhängig von Faktoren wie Geschlecht, Alter oder Ejektionsfraktion ist die Mortalitätsrate bei diastolischer Dysfunktion generell erhöht. Außerdem zeigt sich, dass die oben beschriebenen Stadien prognostische Bedeutung haben. Leichte und mäßige diastolische Dysfunktion gehen mit einer 
geringeren Gesamtmortalität einher als die schwere diastolische Dysfunktion (Redfield et al. 2003). Bei pathologischer E/A-Ratio $(<0,6$ oder $>1,5)$ ist außerdem die kardiale Sterblichkeitsrate um das Zwei- bis Vierfache erhöht (Bella et al. 2002).

Diastolische Funktionsstörungen sind assoziiert mit arteriellem Hypertonus, linksventrikulärer Hypertonie, KHK, hohem BMI und Diabetes mellitus (Fischer et al. 2003). Außerdem ist bei niedriger oder überhöhter E/A-Ratio das Risiko für die Entwicklung einer Herzinsuffizienz erhöht (Aurigemma et al. 2001).

\subsection{Diabetes und diastolische Dysfunktion}

Bei der Erkrankung des Diabetes mellitus nimmt die diastolische Dysfunktion eine wichtige Rolle ein. Die Strong-Heart-Studie zeigte, dass eine Assoziation eines Diabetes mellitus mit linksventrikulärer Hypertrophie und einer diastolischen Dysfunktion besteht (Bella et al. 2002). Eine diastolische Dysfunktion bei Diabetikern ist häufig, ihre Prävalenz lag in Studien bei $60 \%$ bis $80 \%$ (Poirier et al. 2001, Bella et al. 2002, Boyer et al. 2004).

Die diastolische Dysfunktion tritt dabei keineswegs erst nach Jahren der Diabeteserkrankung auf. Sie ist vielmehr ein Frühzeichen der diabetischen Kardiomyopathie und entsteht bevor eine Störung der systolischen Ventrikelfunktion nachweisbar ist (Ohno M et al. 1994). Schon bei neu diagnostizierten Typ-2Diabetikern ohne eine klinisch manifeste Herzerkrankung besteht eine höhere linksventrikuläre Masse und diastolische Funktionsstörung (Vanninen et al. 1992; Di Bonito et al. 1996). Sowohl klinische Studien als auch Studien am Tiermodell zeigten, dass eine Störung der diastolischen Funktion bereits bei Vorliegen einer abnormen Nüchternglucose auftritt (Celentano et al. 1995; Mizushige et al. 2000). In diesem Zusammenhang scheint eine schon vor der Manifestation eines Diabetes bestehende Insulinresistenz eine Rolle zu spielen (Ingelsson et al. 2005).

Die Erkennung einer diastolischen Dysfunktion in einem frühen Stadium der Erkrankung wäre wünschenswert, um die Entwicklung einer symptomatischen Herzinsuffizienz zu verhindern. Das Risiko als Diabetiker eine Herzinsuffizienz zu entwickeln ist für Männer 2,4-fach, für Frauen sogar 5-fach erhöht (Kannel et al. 1974). Patienten, die an einer diastolischen Herzinsuffizienz leiden haben im Vergleich zu Gesunden doppelt so häufig einen Diabetes mellitus (Kitzman et al. 2001). 
Die linksventrikuläre diastolische Funktion kann nicht-invasiv mittels DopplerEchokardiographie beurteilt werden und wird von der European Society of Cardiology als primäres diagnostisches Mittel empfohlen (European Study Group on Diastolic Heart Failure 1998). Seit Kurzem existieren neuere Techniken wie der Gewebedoppler, welche eine validere Beurteilung der diastolischen Funktion zulassen und es uns ermöglichten, den Zusammenhang von Glucosestoffwechsel und diastolischer Dysfunktion genauer zu analysieren.

\subsection{Fragestellung}

In dieser Arbeit soll der Zusammenhang zwischen Diabetes mellitus und einer diastolischen Dysfunktion erörtert werden. Insbesondere wird untersucht, ob eine Assoziation zwischen Güte der Blutzuckereinstellung sowie Schweregrad der Diabeteserkrankung (NIDDM versus IDDM) und einzelnen echokardiographischen Parametern der diastolischen Funktion besteht. 


\section{Material und Methoden}

\subsection{Patientenrekrutierung}

Im Rahmen der Studie „Prävalenz und Verlauf der diastolischen Dysfunktion und der diastolischen Herzinsuffizienz" (Diast-CHF) an der Universitätsmedizin Göttingen wurden innerhalb des Teilprojektes 7 (TP7) „Diastolische Dysfunktion des Kompetenznetzes Herzinsuffizienz (KNHI)“ 1735 Patienten im Zeitraum vom 05.07.2004 bis zum 22.12.2006 rekrutiert. Die prospektive, multizentrische Kohortenstudie wurde ebenfalls in weiteren Studienzentren in Berlin, Lübeck, Marburg, Würzburg und Essen durchgeführt.

Der Einschluss der Patienten erfolgte über die elektronische Datenverarbeitung von hausärztlichen Praxen beziehungsweise den Basisdatensatz der Abteilung Allgemeinmedizin der Universitätsmedizin Göttingen. Die Auswahl der Teilnehmer wurde anhand eines anonymisierten Datenreports aus den Jahren 2000 und 2001 durchgeführt. Eingeschlossen wurden Patienten mit den unten näher beschriebenen kardiovaskulären Risikofaktoren oder einer bekannten Herzerkrankung. Diese Faktoren wurden mittels Freitext oder ICD-Kodierung erfasst (Giere 2000). Im Genaueren können die Kriterien für die Suche im Freitext in Tabelle 1 und die ICDKriterien in Tabelle 2 nachvollzogen werden. 


\begin{tabular}{|l|l|}
\hline Eingegebener Suchbegriff & Gesuchte Diagnose \\
\hline${ }^{*}$ iabete* & Diabetes mellitus \\
${ }^{*}$ luthoch* & Bluthochdruck \\
${ }^{*}$ yperton* & Hypertonus, Hypertonie \\
${ }^{*}$ yperten* & Hypertensiv(e) \\
${ }^{*}$ infar* & Herzinfarkt, Myokardinfarkt, Hirninfarkt \\
${ }^{*}$ erzkrank $^{*}$ & Koronare Herzkrankheit \\
${ }^{*}$ ngina *ector* & Angina pectoris \\
${ }^{*}$ erzinsuff* & Herzinsuffizienz \\
${ }^{*}$ erzschwäch* & Herzschwäche \\
${ }^{*}$ plex* & Apoplex \\
${ }^{*}$ chlaganfal* & Schlaganfall \\
pAVK & pAVK \\
pavk & pAVK \\
paVk & pAVK \\
${ }^{*}$ erschlußkrank ${ }^{*}$ & Periphere arterielle Verschlusskrankheit \\
${ }^{*}$ chlafapno* & Schlaf-Apnoe-Syndrom \\
\hline
\end{tabular}

Tab. 1: Kriterien der Freitextsuche

\begin{tabular}{|l|l|}
\hline Eingegebener ICD-Code & Gesuchte Diagnose \\
\hline E11.* & Diabetes mellitus \\
G47.3 & Schlaf-Apnoe-Syndrom \\
$11^{*} .^{*}$ & Hypertonie \\
$121 .^{*}$ & Ischämische Herzkrankheiten \\
$122 .^{*}$ & Ischämische Herzkrankheiten \\
$123 .^{*}$ & Ischämische Herzkrankheiten \\
$125 .^{*}$ & Ischämische Herzkrankheiten \\
$150 .^{*}$ & Herzinsuffizienz \\
$163 .^{*}$ & Hirninfarkt \\
$165 .{ }^{*}$ & Schlaganfall \\
$166 .{ }^{*}$ & Verschluss/ Stenose präcerebr. Arterien \\
$169 .{ }^{*}$ & Z.n. cerebrovaskulärem Ereignis \\
173.9 & Periphere arterielle Verschlusskrankheit \\
\hline
\end{tabular}

Tab. 2: Kriterien zur Suche nach ICD-Diagnosen (Giere 2000) 


\subsection{Ein- und Ausschlusskriterien}

In die Studie eingeschlossen wurden Patienten im Alter zwischen 50 und 85 Jahren. Vom Hausarzt musste entweder ein Risikofaktor für diastolische Herzinsuffizienz in Form eines arteriellen Hypertonus, eines Schlaf-Apnoe-Syndroms oder einer manifesten Arteriosklerose dokumentiert werden oder aber eine manifeste Herzinsuffizienz bekannt sein. Vom Vorliegen einer Herzinsuffizienz wurde ausgegangen, wenn ein Arzt bereits eine Herzinsuffizienz diagnostiziert hatte oder aber wenn mindestens ein Haupt- und zwei Nebenkriterien gemäß der FraminghamStudie vorhanden waren (McKee et al. 1971). Außerdem war eine schriftliche Einverständniserklärung des Patienten zur Studienteilnahme erforderlich.

Für die Analysen dieser Arbeit wurden nur diejenigen Patienten berücksichtigt, die bei der ersten Untersuchung einen oralen Glucosetoleranztest erhalten hatten oder bei denen bereits ein Diabetes mellitus Typ II bekannt war. Patienten, bei denen ein Diabetes mellitus vor dem 40. Lebensjahr diagnostiziert worden war und welche innerhalb eines Jahres insulinpflichtig wurden, wurden als Typ-I-Diabetiker klassifiziert und von den Analysen ausgeschlossen.

Patienten, bei denen aufgrund schlechter Deutschkenntnisse, einer Aphasie oder anderer Sprachstörungen erhebliche Kommunikationsschwierigkeiten bestanden, wurden ebenfalls von der Studienteilnahme ausgeschlossen. Auch Erkrankungen, die möglicherweise die Einwilligungsfähigkeit der Patienten beeinträchtigten oder eine mangelnde Kooperationsbereitschaft waren Ausschlusskriterien. Schließlich wurden Patienten von der Studie ausgeschlossen, bei denen aus geographischen Gründen eine Studienteilnahme nicht möglich war.

\subsection{Durchführung und Datenerhebung}

Der Untersuchungszeitraum betrug drei Jahre pro Patient. Die Patienten kamen innerhalb dieses Zeitraumes in jährlichen Abständen zur Untersuchung. Diese beinhaltete eine Anamnese, körperliche Untersuchung, Labortests, eine echokardiographische Untersuchung, ein EKG, ein 6-Minuten-Gehtest sowie ein Fragebogen bezüglich Lebensqualität und psychosozialer Hintergründe. Patienten, denen es aus gesundheitlichen oder anderen Gründen, wie zum Beispiel einem Wohnortwechsel nicht möglich war, zur Untersuchung zu erscheinen, wurden 
telefonisch bezüglich ihres Gesundheitszustandes, eventuell neu aufgetretener Erkrankungen, kardiovaskulärer Ereignisse und aktueller Medikation befragt.

Bezüglich des Diabetes mellitus wurde erfasst, seit wann die Erkrankung bestand oder ob sie im Untersuchungszeitraum neu aufgetreten war. Bei Diabetikern wurden außerdem die Art und eventuelle Änderungen der antidiabetischen Therapie erhoben. Insbesondere wurde erfasst, ob die Diabetestherapie mittels diätischer Maßnahmen, oraler Antidiabetika und oder Insulin erfolgte. Auch die Anzahl der gespritzten Insulineinheiten pro Tag wurde erhoben.

Bei allen Patienten, bei denen ein Diabetes mellitus bestand oder die einen oGTT erhielten, wurde in der Baseline-Untersuchung außerdem der HbA1c-Wert bestimmt. Dieser wurde in EDTA-Blut mit einem Integra 800® Analysator (Roche Diagnostics, Mannheim, Deutschland) gemessen. Der Referenzbereich lag hier bei 2,9 - 4,2\%.

\subsection{Diagnostik}

\subsubsection{Diabetes mellitus und oraler Glucosetoleranztest}

Ein Diabetes mellitus galt als diagnostiziert, wenn der Patient antidiabetische Medikation einnahm, vom Hausarzt die Diagnose ,Diabetes mellitus' dokumentiert worden war oder ein entsprechendes oGTT-Ergebnis vorlag.

Der orale Glucosetoleranztest wurde entsprechend eines vereinfachten Protokolls bei 627 Patienten ohne bekannten Diabetes mellitus durchgeführt. Der Patient durfte vor oder während des Tests nicht rauchen und saß oder lag während der Durchführung. Die Plasmaglucose wurde zunächst in nüchternem Zustand bestimmt. Dann trank der Patient $75 \mathrm{~g}$ Glucose in 250-300ml Wasser innerhalb von fünf Minuten. Nach 60 und 120 Minuten erfolgte eine Blutentnahme zwecks Glucosebestimmung. Das Plasma Insulin wurde im nüchternen Zustand und zwei Stunden nach der Glucosezufuhr bestimmt.

Die Interpretation der Ergebnisse erfolgte in Übereinstimmung mit den aktuellen Empfehlungen der ADA (American Diabetes Association 2009). Ein normaler Glucosestoffwechsel galt somit als gesichert, wenn der Glucosewert in nüchternem Zustand $<100 \mathrm{mg} / \mathrm{dl}$ und der 2-Stunden-Glucosewert $<140 \mathrm{mg} / \mathrm{dl}$ war. Von einem Prädiabetes wurde bei einem Nüchtern-Glucosewert von 100-125mg/dl und einem 2Stunden-Glucosewert von 140-199 mg/dl ausgegangen. Bei einem Nüchtern- 
Glucosewert von $>125 \mathrm{mg} / \mathrm{dl}$ und einem $2 \mathrm{~h}$ Glucosewert im venösen Plasma von $\geq 199 \mathrm{mg} / \mathrm{dl}$ (11,1 mmol/l) wurde ein Diabetes mellitus angenommen.

Die Insulinbestimmung erfolgte mit einem ADVIA Centaur ${ }^{\circledR}$ (früher Bayer Diagnostics, jetzt Siemens Health Care Diagnostics, Eschborn, Deutschland) mittels Immunoassay.

\subsubsection{Echokardiographie}

Die echokardiographische Untersuchung erfolgte mit einem Hewlett-Packard Sonos 5500 Gerät (Hewlett Packard, Andover, MA, USA) gemäß den Kriterien der American Society of Cardiology (ASE) (Schiller et al. 1989; Quinones et al. 2002; Cheitlin et al. 2003). Nach einer mindestens 10-minütigen Ruhepause des Patienten wurde die Untersuchung von einem erfahrenen Untersucher durchgeführt. Während der Untersuchung befand sich der Patient in klassischer Linksseitenlage mit $30^{\circ}$ angehobenem Oberkörper und hinter dem Kopf gelagertem linken Arm. Es erfolgte eine umfassende echokardiographische Untersuchung insbesondere der diastolischen Funktion, bei der neben B-Bild, kontinuierlichem (cw) und gepulstem (pw) Doppler auch Gewebedoppler und M-Mode zur Anwendung kamen. Während der gesamten Untersuchung wurde ein EKG abgeleitet.

Vor Messung der diastolischen Parameter wurde im parasternalen Langachsenschnitt mittels M-Mode der aortale Durchmesser in Klappenebene, linksventrikulärer enddiastolischer und endsystolischer Durchmesser (LVD) und linksatrialer endsystolischer Durchmesser (LAD) gemessen. Außerdem bestimmt wurden enddiastolische interventrikuläre Septumdicke (IVS) sowie enddiastolische Hinterwanddicke (PWD).

\subsubsection{Füllungsvolumina und Ejektionsfraktion}

Um die Ejektionsfraktion (EF), die linksventrikulären endsystolischen und enddiastolischen Füllungsvolumina (LVV) zu bestimmen, wurde im konventionellen, zweidimensionalen Vier-Kammer-Blick von apikal die Scheibchensummationsmethode (modifizierte Simpson-Regel) angewendet. Bei dieser Methode wird der irregulären Form des linken Cavums insofern Rechnung getragen, als der Ventrikel rechnerisch in eine bestimmte Anzahl ellipsenförmiger Zylinder aufgeteilt wird. Im Vier-Kammer-Blick und (im 90 Winkel dazu) im ZweiKammer-Blick wird die Kontur des Endokards nachgezeichnet und somit die Fläche 
der einzelnen Zylinder definiert. Durch Summation der Zylindervolumen erhält man das endsystolische und enddiastolische Volumen und kann prozentual daraus die Ejektionsfraktion bestimmen (Tortoledo et al. 1983):

$$
E F=\frac{\text { LVEDV }- \text { LVESV }}{\text { LVEDV }} \times 100 \%
$$

Die linksatrialen endsystolischen Durchmesser (LAD) wurden ebenfalls im VierKammer-Blick von apikal jeweils einmal quer und einmal längst gemessen.

Zudem wurden im Vier-Kammer-Blick eventuell beobachtete Wandbewegungsstörungen, rechtsventrikuläre Dilatation, Perikardergüsse oder eine gestaute Vena cava inferior dokumentiert.

\subsubsection{Mitrales Einstromprofil}

Zur Beurteilung des mitralen Einstromprofils wurden im apikalen Vier-Kammer-Blick mittels gepulsten Dopplers über der Mitralklappe früh- (E-Welle) und spätdiastolische (A-Welle) Geschwindigkeitsmaxima abgeleitet. Die E-Wellen-Dezelerationszeit (EDCT) wurde als Zeitspanne vom Erreichen der Maximalgeschwindigkeit der EWelle bis zur Kreuzung mit der Nulllinie einer an die Dezeleration angelegten Tangente ermittelt (Nagueh et al. 1997). Die Messung von E- und A-Welle sowie der Dezelerationszeit erfolgte anschließend ebenfalls mit Valsalva-Doppler um eine eventuelle Pseudonormalisation zu demaskieren.

\subsubsection{Isovolumetrische Relaxationszeit}

Um die isovolumetrische Relaxationszeit (IVRT) zu bestimmen, wurde der CWDoppler im Fünf-Kammer-Blick so zwischen Mitralklappe und linksventrikulären Ausflusstrakt gelegt, dass sowohl E- und A-Welle als auch der systolische aortale Ausstrom erfasst wurden. Die IVRT entspricht dem Zeitintervall zwischen Aortenklappenschluss und Mitralklappenöffnung, im Flussprofil repräsentiert durch die Zeit zwischen Sistieren des transaortalen Ausflusses und Beginn des transmitralen Einstromes.

Die IVRT ist jedoch abhängig von Herzfrequenz und Vorlast. Zudem kann bei Normwerten der IVRT trotzdem eine verlangsamte isovolumetrische Relaxation vorliegen, da ein erhöhter linksatrialer Druck zu einer vorzeitigen Mitralklappenöffnung führt (Myreng und Smiseth 1990). 


\subsubsection{Mitralanulusgeschwindigkeiten}

Das Messvolumen des pw-Gewebedopplers wurde auf Höhe des lateralen Mitralanulus platziert. Mit der Spektraleinstellung ließen sich die anterograde systolische Welle (S') und die jeweils retrograde frühdiastolische $E^{\prime}$ - und spätdiastolische A'-Welle ermitteln.

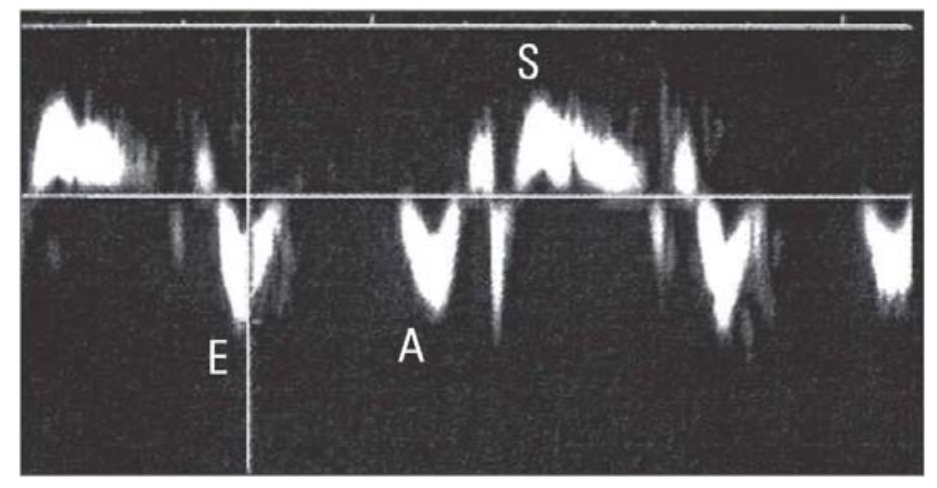

Abb. 1: S', E'- und A'-Welle im Gewebedoppler (hier mit E, A und S bezeichnet); Quelle: Grausenburger 2006, S.222

\subsubsection{Pulmonalvenenflussprofil}

Die Bedeutung der Untersuchung des Pulmonalvenenflusses für die Abschätzung des linken Vorhofdruckes ist durch Studien belegt (Kuecherer et al. 1990). Im apikalen Vier-Kammer-Blick wurde mittels Farbdoppler die rechte Pulmonalvene dargestellt. Der gepulste Doppler wurde in das Flusssignal der Pulmonalvene gelegt und das Pulmonalvenenflussprofil abgeleitet. Die S-Welle reflektiert den Fluss während der Systole (PVSF), die D-Welle den diastolischen Fluss in den Pulmonalvenen (PVDF). Die umgekehrt gerichtete AR-Welle (atrialer Rückfluss) entsteht durch die Kontraktion des Vorhofs (PVAF).

\subsubsection{Beurteilung und Klassifikation der diastolischen Dysfunktion}

Die linksventrikuläre Masse (LVM) wurde entsprechend der Formel von Devereux berechnet (Devereux et al. 1986):

$L V$-Masse $[g]=1,04 \times(L V E D D+I V S D+L V P W D)^{3}-(L V E D D)^{3} \times 0,8+0,6$

Hierbei steht 1,04 für das spezifische Gewicht des Myokards, LVEDD bezeichnet den enddiastolischen Ventrikeldurchmesser, IVSD den enddiastolischen Durchmesser des Ventrikelseptums und PWD den enddiastolischen Durchmesser der Hinterwand. 
Die Muskelmasse wurde auf die Körperoberfläche (KOF) normiert und ergab somit den Muskelmassenindex: $L V M I=L V M / K O F$.

Zur Beurteilung der diastolischen Funktionen wurden die E/A- und die S/D-Ratio sowie die E/e'- und e'/a'-Ratio berechnet.

Anschließend wurde die Einteilung der diastolischen Dysfunktion in die verschiedenen Schweregrade folgendermaßen vorgenommen:

\begin{tabular}{|c|c|c|c|c|}
\hline & $\begin{array}{l}\text { normal } \\
\text { Grad } 0\end{array}$ & $\begin{array}{c}\text { mild } \\
\text { Grad } 1\end{array}$ & $\begin{array}{c}\text { moderat } \\
\text { Grad } 2\end{array}$ & $\begin{array}{l}\text { schwer } \\
\text { Grad } 3\end{array}$ \\
\hline EIA & $\geq 1$ & $<1$ & $\geq 1 \&<2$ & $\geq 2$ \\
\hline & & & $\begin{array}{l}\text { + einen der folgenden } \\
\text { Werte: }\end{array}$ & $\begin{array}{c}\text { + einen der folgenden } \\
\text { Werte }\end{array}$ \\
\hline Ele' & $<10$ & & $\geq 10$ oder & $\geq 10$ \\
\hline S/D & $\geq 1$ & & $<1$ oder & $<1$ \\
\hline $\begin{array}{l}\text { ElA } \\
\text { mit Valsalva- } \\
\text { Manöver }\end{array}$ & $\geq 1$ & & $<1$ & \\
\hline
\end{tabular}

Tab. 3: Einteilung des Schweregrades der diastolischen Dysfunktion

\subsubsection{6-Minuten-Gehtest}

Der 6-Minuten-Gehtest ist ein einfaches, gleichwohl valides und reliables diagnostisches Mittel zur Beurteilung der Leistungsfähigkeit kardiologischer und pulmonologischer Patienten (Guyatt et al. 1985). Ihm wird neben der diagnostischen auch eine prognostische Bedeutung hinsichtlich Morbidität und Mortalität beigemessen (Castel et al. 2009). Bei standardisierter Durchführung ist er sehr gut reproduzierbar (O'Keeffe et al. 1998).

Der Test wurde entsprechend der ATS-Leitlinien (American Thoracic Society 2002) durchgeführt. Der Patient sollte innerhalb von sechs Minuten die für ihn größtmögliche Strecke zurücklegen, ohne dabei zu laufen oder zu rennen. Ein Fuß hatte stets auf dem Boden zu bleiben. Der Versuchsleiter blieb während der Testung am Anfang der Teststrecke stehen. Der Teilnehmer konnte seine 
Gehgeschwindigkeit selbst bestimmen und bei Bedarf auch Pausen einlegen, indem er stehen blieb oder sich hinsetzte. Währenddessen wurde die Zeitmessung nicht unterbrochen. Der Patient wurde vom Versuchsleiter ermutigt, weiterzugehen sobald er sich wieder dazu in der Lage fühlte. Erschien es dem Patienten jedoch unmöglich mit dem Gehen fortzufahren, so konnte er den Test von sich aus abbrechen. Zeitpunkt und Grund des Abbruches wurden dokumentiert.

Der Versuchsleiter ermutigte den Patienten alle 30 Sekunden mit folgenden Formulierungen: „Das machen Sie gut.“ „Immer weiter so.“ Alle zwei Minuten sagte der Versuchsleiter die verbleibende Zeit an. Nach Beendigung des Tests wurde die zurückgelegte Distanz, auf ganze Meter gerundet, dokumentiert.

\subsection{Statistik}

\subsubsection{Deskriptive Statistik}

Zur Darstellung und Veranschaulichung der Daten wurden Methoden der deskriptiven Statistik verwendet. Die Patienten wurden nach den oben genannten Kriterien in die folgenden vier Schweregrade der Glucosestoffwechsel-Störung eingeteilt: ,Normal', ,Prädiabetes', ,NIDDM' und ,IDDM'. Im Zuge dieser Arbeit soll zunächst ausschließlich ein Vergleich der Diabetesgruppen mit den Nicht-Diabetikern erfolgen. Bei signifikanten Ergebnissen wird der Aspekt des Prädiabetes als Zwischenstufe zwischen normaler Glucosehomöostase und manifestem Diabetes mellitus Gegenstand weiterführender Arbeiten sein.

Einige Variablen erwiesen sich als nicht normalverteilt, sodass eine Berechnung des Mittelwertes nicht sinnvoll erschien. Es wird daher bei allen stetigen Variablen der Median sowie der Interquartilbereich angegeben. Für kategorische Variablen werden absolute Zahlen und relative Prozentsätze angegeben.

Die statistischen Analysen wurden mit SPSS Statistics 17.0 Software durchgeführt. Signifikanz wurde ab einem $\mathrm{P}$-Wert von $<0,05$ angenommen.

\subsubsection{Analytische Statistik}

Stetige Variablen, die stark von einer Normalverteilung abwichen, wurden vor Durchführung der Tests logarithmiert. 
Der Jonckheere-Terpstra-Test ist ein nichtparametrischer statistischer Test, mittels welchem sich vergleichen lässt, ob sich verschiedene unabhängige Stichproben (in diesem Fall die Gruppen der Glucosestoffwechsel-Störung) hinsichtlich einer ordinalskalierten Variable unterscheiden. Basierend auf der Annahme, dass die Medianwerte einer Rangordnung folgen, lässt sich somit die Hypothese $\mathrm{H} 1$ wie folgt formulieren: $M 1 \leq \mathrm{M} 2 \leq \ldots \leq \mathrm{Mi}$. $\mathrm{M}$ steht hierbei für den jeweiligen Median der Stichprobe. Mindestens ein $\leq$ sollte durch ein < ersetzt werden können. Die Nullhypothese Ho lautet demzufolge: Die Populationsmediane weisen keine Unterschiede auf: M1=M2 $=\ldots=$ Mi. Der Jonckheere-Terpstra-Test testet also auf das Vorliegen eines Trends zwischen den Gruppen. Voraussetzung für den Test ist das Vorliegen von Stichproben aus homomeren (formgleich verteilten) Populationen (Terpstra 1952; Jonckheere 1954; Bewick et al. 2004).

Um zu untersuchen, ob innerhalb bestimmter Gruppen (hier: Gruppen der Glucosehomöostase) ein Trend für kategoriale Variablen zu verzeichnen war, wurde der Cochran-Armitage-Trend-Test angewendet. Der Test prüft ob für eine binomiale Variable eine dosisabhängige Beziehung besteht (Cochran 1954; Armitage 1955; Corcoran und Mehta 2002).

Für die Untersuchung von Zusammenhängen zwischen einzelnen Parametern wurden bivariate Korrelationsanalysen durchgeführt. Um das Zusammenwirken mehrerer Variablen zu untersuchen, wurden eine multivariate Kovarianzanalyse sowie eine bedingte schrittweise logistische Regressionsanalyse durchgeführt. Bei der logistischen Regression wird die Wahrscheinlichkeit der Zugehörigkeit zu einer Gruppe in Abhängigkeit von einer oder mehrerer unabhängiger Variablen bestimmt. Bei der hier verwendeten schrittweisen Methode wird zunächst die erklärungsstärkste, unabhängige Variable aufgenommen (Vorwärtsselektion) bzw. die erklärungsschwächste aus dem vollständigen Modell entfernt (Rückwärtsselektion). 


\section{Ergebnisse}

\subsection{Patientenkollektiv}

Insgesamt wurden die Daten von 1085 Patienten für die vorliegenden Ergebnisse analysiert. Von den untersuchten Patienten waren $46 \%$ Frauen. Das durchschnittliche Alter der Patienten betrug 66 Jahre.

Bei 465 Patienten war zum Zeitpunkt des Studieneinschlusses ein Diabetes mellitus bekannt. Von diesen wurden vier Patienten mit Diabetes mellitus Typ 1 aus den Analysen ausgeschlossen. Die Verteilung der untersuchten Patienten über die vier Schweregrade der Glucosestoffwechsel-Störung kann anhand der unten gezeigten Graphik nachvollzogen werden (Abb. 2). Die größte Gruppe stellte die Gruppe der Patienten mit normaler Glucosehomöostase mit insgesamt 343 Patienten dar, gefolgt von der Gruppe der nicht-insulinpflichtigen Diabetiker (NIDDM) mit 335 Patienten. Diese Gruppe beinhaltete sowohl die Patienten mit vorbekanntem NIDDM bei Studieneinschluss (283 Patienten) als auch die Patienten, bei denen im Rahmen des oGTTs der Baseline-Untersuchung ein Diabetes mellitus neu diagnostiziert worden war (52 Patienten). 229 Patienten hatten einen Prädiabetes. Die kleinste Gruppe bildeten schließlich die insulinabhängigen Diabetiker (178 Patienten). Männer und Frauen waren in den jeweiligen Glucosestoffwechsel-Gruppen ungefähr zu gleichen Teilen repräsentiert (Anteil der Männer: Normale Glucosehomöostase: 47,2\%; Prädiabetes: 54,1\%; IDDM: 51,7\%). Eine Ausnahme stellte diesbezüglich die Gruppe der nicht-insulinabhängigen Diabetiker dar, in der männliche Patienten mit 61,2\% einen größeren Anteil ausmachten. 


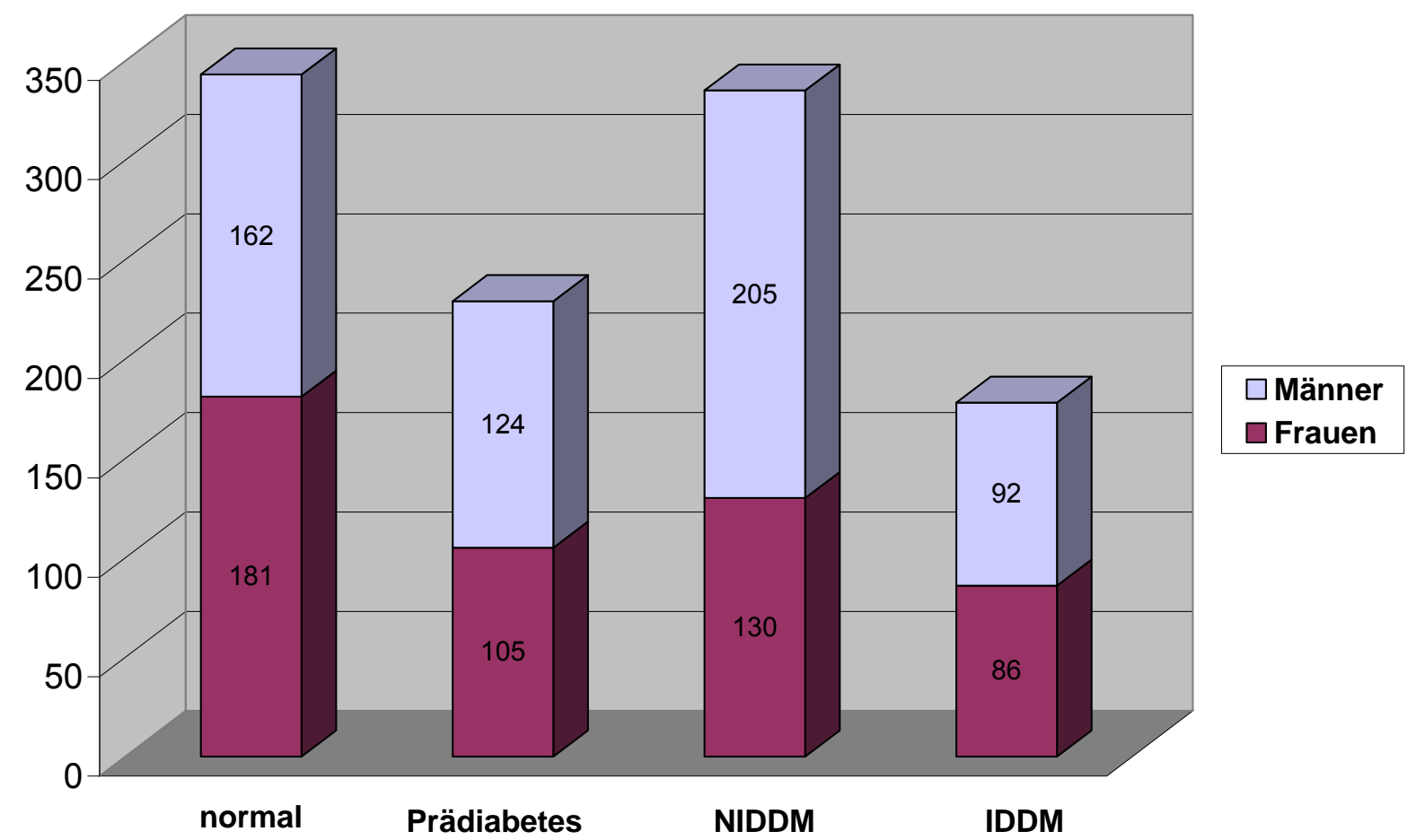

Abb. 2: Verteilung des Patientenkollektivs auf die verschiedenen Schweregrade der Glucosestoffwechsel-Störung

\subsubsection{Oraler Glucosetoleranztest}

637 Patienten erhielten während der Baseline-Untersuchung einen oralen Glucosetoleranztest. Bei Patienten mit insulinpflichtigem Diabetes mellitus (IDDM) wurde prinzipiell kein oGTT durchgeführt. Nur ein kleiner Teil der Patienten mit einem schon bekannten nicht-insulinabhängigen Diabetes mellitus (NIDDM) durchlief eine orale Glucosetoleranztestung (insgesamt 13 Patienten).

Die Ergebnisse des oralen Glucosebelastungstests stellten sich wie folgt dar: Von den untersuchten 637 Patienten wiesen 343 einen normalen Glucosestoffwechsel und 229 einen Prädiabetes auf, während bei 52 Patienten ein Diabetes mellitus neu diagnostiziert wurde (vgl. Abb. 3). 


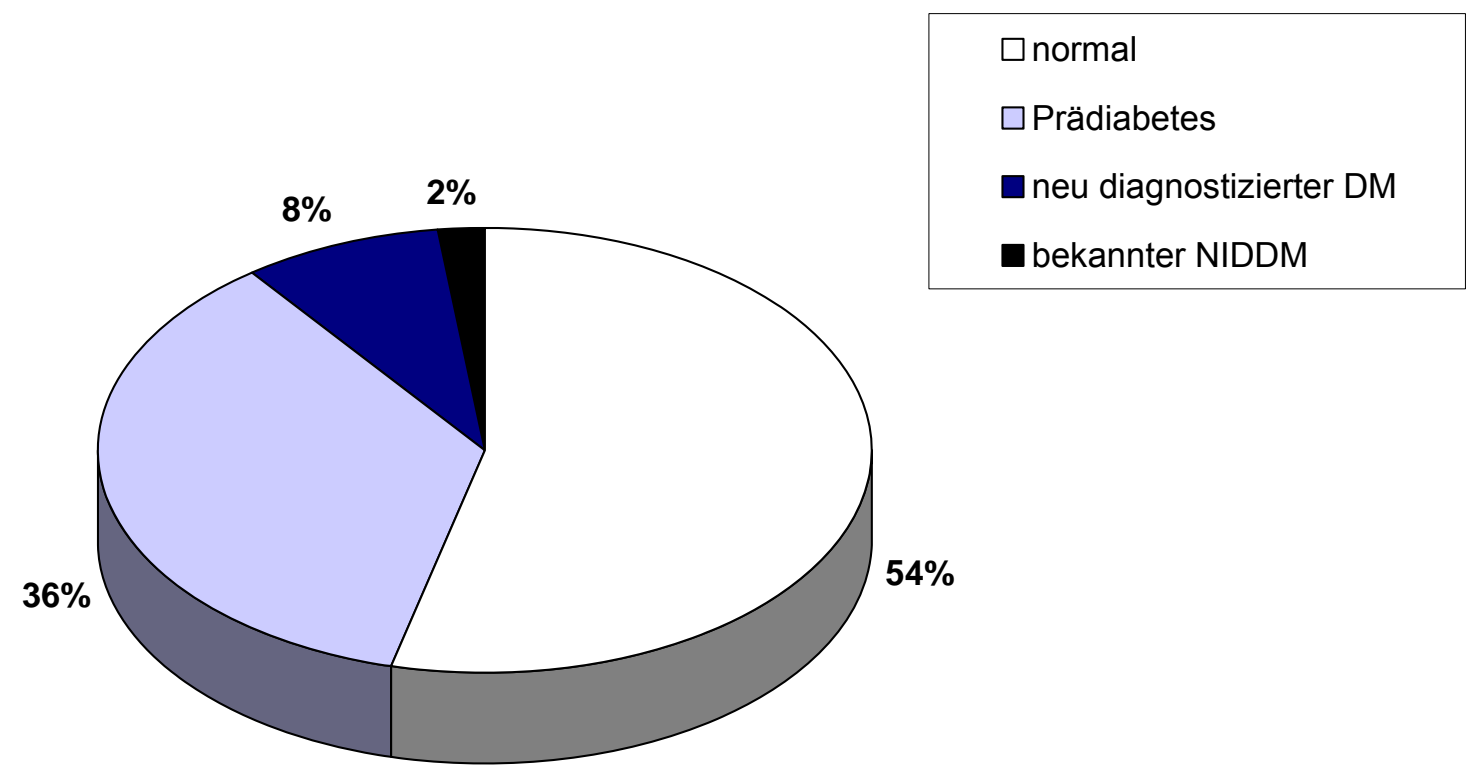

Abb. 3: Ergebnisse des OGTT: Prozentuale Verteilung

Im Folgenden sollen nun insbesondere Nicht-Diabetiker und Diabetiker, also die Gruppen 1) Patienten mit normalem Glucosestoffwechsel 2) nicht-insulinpflichtige Diabetiker (NIDDM) und 3) insulinpflichtige Diabetiker (IDDM) miteinander verglichen werden (insgesamt 856 Patienten).

\subsubsection{Allgemeine Charakteristika der einzelnen Gruppen}

Die nachfolgende Tabelle (Tab. 4) zeigt die grundlegenden Charakteristika der jeweiligen Gruppen der Glucosestoffwechsel-Störung. Angegeben ist jeweils der Median und der Interquartilbereich in eckigen Klammern. Die p-Wert-Berechnung erfolgte mittels des oben beschriebenen Jonckheere-Terpstra-Tests. 


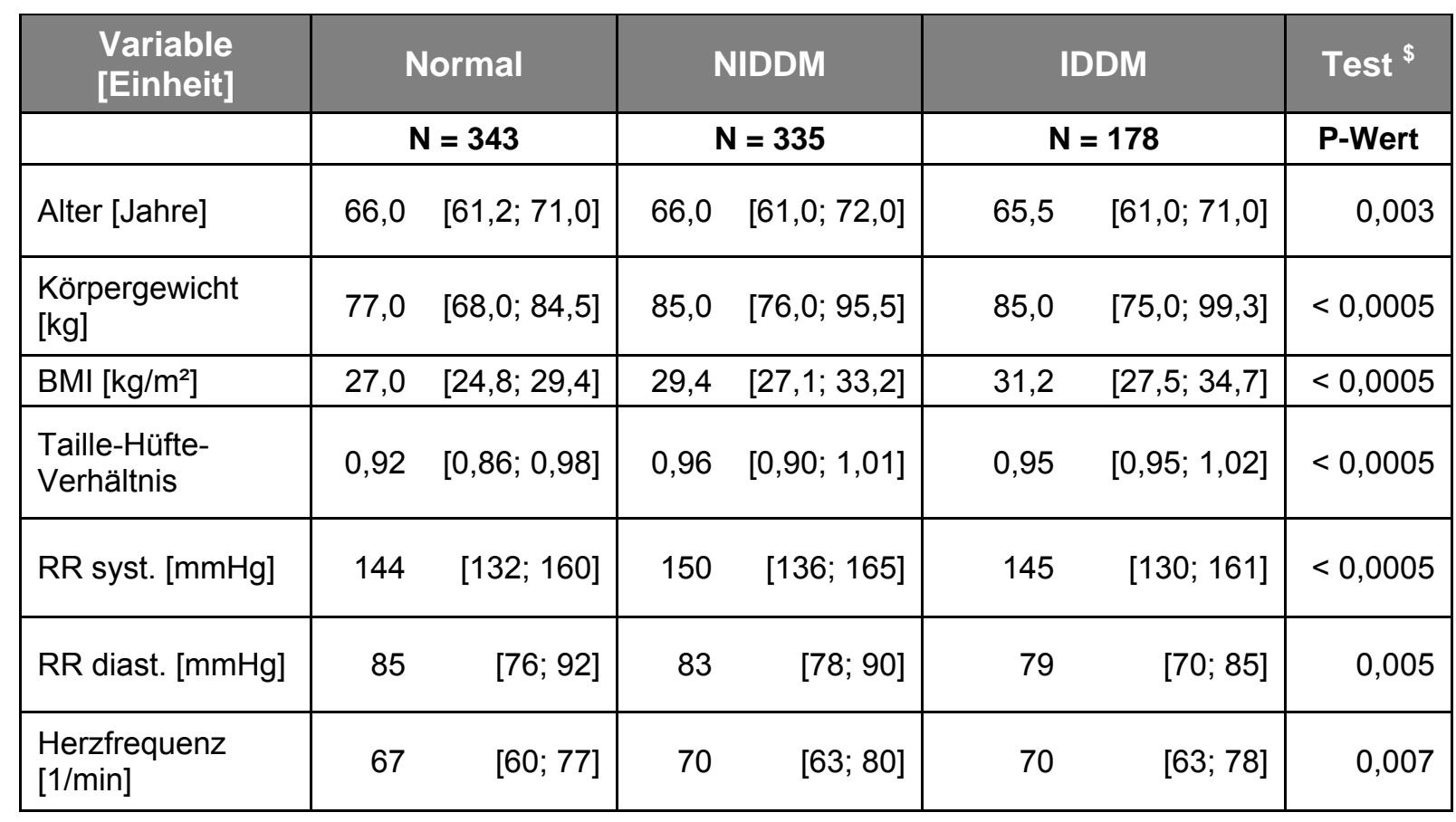

n.s. = nicht signifikant

\$ Jonckheere-Terpstra-Test

Tab. 4: Allgemeine Charakteristika der Gruppen. Median und [Interquartilbereich].

Bezüglich des Alters, der Herzfrequenz und des diastolischen Blutdruckes waren schwach signifikante Trends über die einzelnen Gruppen festzustellen. Bei Diabetikern (NIDDM und IDDM) lag der Median des Körpergewichts um 8kg höher als bei Nicht-Diabetikern, zudem hatten sie ein größeres Taille-Hüfte-Verhältnis (THV). Auch der BMI war dementsprechend bei insulinabhängigen Diabetikern mit einem Median von 31,2 am höchsten und bei Patienten mit normalem Glucosestoffwechsel am niedrigsten (Median = 27,0). Die Werte des diastolischen Blutdruckes verringerten sich von Nicht-Diabetikern mit einem Medianwert von $85 \mathrm{mmHg}$ über $83 \mathrm{mmHg}$ bei nicht-insulinabhängigen bis hin zu $79 \mathrm{mmHg}$ bei insulinpflichtigen Diabetikern.

Für die beiden Diabetesgruppen wurde erhoben, wie lange die Diabeteserkrankung bestand (Tab. 5). Wie zu erwarten, wiesen insulinpflichtige Diabetiker eine längere Erkrankungsdauer auf (Median 13 Jahre) als Diabetes-Patienten, die noch keine Insulintherapie benötigten (Median 4 Jahre). Der Median der täglich injizierten Insulineinheiten der insulinpflichtigen Diabetiker lag bei 48IE. 


\begin{tabular}{|c|c|c|c|c|}
\hline Variable [Einheit] & NIDDM & \multicolumn{2}{|c|}{ IDDM } & Test $^{\$}$ \\
\hline & $N=335$ & \multicolumn{2}{|c|}{$N=178$} & P-Wert \\
\hline Dauer des Diabetes [Jahre] & $4 \quad[1 ; 7,3]$ & 13 & {$[6,8 ; 20,0]$} & $<0.0005$ \\
\hline Dauer der Insulinabhängigkeit [Jahre] & - & & {$[2,0 ; 8,5]$} & - \\
\hline Insulineinheiten pro Tag [IE] & - & 48 & {$[34 ; 76]$} & - \\
\hline
\end{tabular}

\$ Jonckheere-Terpstra-Test

Tab. 5: Charakteristika der Diabetesformen. Median und [Interquartilbereich]

\subsubsection{Komorbiditäten und Risikofaktoren}

Die drei Gruppen wurden hinsichtlich der Prävalenz von Komorbiditäten und Risikofaktoren für eine diastolische Dysfunktion untersucht. Tabelle 6 zeigt die Ergebnisse der Analysen, angegeben sind jeweils die absoluten Zahlen und die Prozentsätze in runden Klammern.

\begin{tabular}{|l|rr|rr|rr|r|}
\hline \multicolumn{1}{|c|}{ Variable [Einheit] } & \multicolumn{2}{|c|}{ Normal } & \multicolumn{2}{|c|}{ NIDDM } & \multicolumn{2}{|c|}{ IDDM } & Test $^{2}$ \\
\hline & \multicolumn{2}{|c|}{$\mathbf{N}=\mathbf{3 4 3}$} & \multicolumn{2}{|c|}{$\mathbf{N}=\mathbf{3 3 5}$} & \multicolumn{2}{|c|}{$\mathbf{N}=\mathbf{1 7 8}$} & P-Wert \\
\hline Arterieller Hypertonus & 300 & $(87,5 \%)$ & 287 & $(85,7 \%)$ & 156 & $(87,6 \%)$ & $<0,001$ \\
\hline Hyperlipidämie & 136 & $(39,7 \%)$ & 166 & $(49,6 \%)$ & 104 & $(58,4 \%)$ & $<0,001$ \\
\hline Raucher & 44 & $(12,8 \%)$ & 38 & $(11,3 \%)$ & 19 & $(10,4 \%)$ & 0,023 \\
\hline $\begin{array}{l}\text { Koronare } \\
\text { Herzkrankheit }\end{array}$ & 57 & $(16,6 \%)$ & 75 & $(22,4 \%)$ & 63 & $(35,4 \%)$ & $<0,001$ \\
\hline $\begin{array}{l}\text { Periphere arterielle } \\
\text { Verschlusskrankheit }\end{array}$ & 11 & $(3,2 \%)$ & 19 & $(5,7 \%)$ & 31 & $(17,4 \%)$ & $<0,001$ \\
\hline $\begin{array}{l}\text { Zerebrale } \\
\text { Gefäßkrankheit }\end{array}$ & 26 & $(7,6 \%)$ & 35 & $(10,4 \%)$ & 13 & $(7,3 \%)$ & 0,045 \\
\hline Herzinsuffizienz & 34 & $(9,9 \%)$ & 64 & $(19,1 \%)$ & 54 & $(17,3 \%)$ & $<0,001$ \\
\hline Schlaf-Apnoe-Syndrom & 20 & $(5,8 \%)$ & 24 & $(7,2 \%)$ & 23 & $(12,9 \%)$ & $<0,001$ \\
\hline Vorhofflimmern & 24 & $(7,0 \%)$ & 23 & $(6,9 \%)$ & 16 & $(9,0 \%)$ & n.s. $(0,772)$ \\
\hline
\end{tabular}

n.s. $=$ nicht signifikant $\quad \sim$ Cochran-Armitage-Trendtest

Tab. 6: Komorbititäten und Risikofaktoren. Anzahl und (Prozentsatz). 
Wie hier ersichtlich, ergab sich bei fast allen Risikofaktoren ein Trend über die Gruppen. Bezüglich der Häufigkeit eines arteriellen Hypertonus bestanden marginale Unterschiede zwischen den Gruppen, nicht-insulinpflichtige Diabetiker litten etwas seltener unter arterieller Hypertonie.

Rauchen war der einzige Risikofaktor, welcher bei Patienten mit normalem Glucosestoffwechsel häufiger zu finden war als bei Diabetikern.

Bezüglich der Hyperlipidämie ergab sich ein kontinuierlicher Anstieg über die Gruppen: 39,7\% der Nicht-Diabetiker, 49,6\% der nicht-insulinpflichtigen und 58,4\% der insulinabhängigen Diabetiker wiesen eine Hyperlipidämie auf. Auch KHK, pAVK und ein Schlaf-Apnoe-Syndrom waren am häufigsten in der Gruppe der insulinpflichtigen Diabetiker, am seltensten in der Gruppe der Nicht-Diabetiker zu finden. Eine Herzinsuffizienz war bei den nicht-insulinabhängigen Diabetikern am häufigsten $(19,1 \%)$ festzustellen, gefolgt von den insulinabhängigen Diabetikern mit 17,3\%. Von den Nicht-Diabetikern hatten 9,9\% eine Herzinsuffizienz. Lediglich bezüglich eines Vorhofflimmerns, welches insgesamt eher selten zu finden war, konnte zwischen den Gruppen kein signifikanter Unterschied gefunden werden.

\subsubsection{Medikation}

In der nachstehenden Tabelle (Tab. 7) werden die Unterschiede bezüglich der Medikation der einzelnen Gruppen dargestellt. 


\begin{tabular}{|c|c|c|c|c|c|c|c|}
\hline Variable [Einheit] & \multicolumn{2}{|c|}{ Normal } & \multicolumn{2}{|c|}{ NIDDM } & \multicolumn{2}{|c|}{ IDDM } & Test \\
\hline & \multicolumn{2}{|c|}{$N=343$} & \multicolumn{2}{|c|}{$N=335$} & \multicolumn{2}{|c|}{$N=178$} & P-Wert \\
\hline ACE-Hemmer & 146 & $(42,9 \%)$ & 165 & $(50,0 \%)$ & 108 & $(60,7 \%)$ & $<0,001$ \\
\hline AT1-Rezeptor-Antagonist & 50 & $(14,7 \%)$ & 58 & $(17,6 \%)$ & 43 & $(24,2 \%)$ & $=0,003$ \\
\hline Betablocker & 156 & $(45,9 \%)$ & 150 & $(45,5 \%)$ & 106 & $(59,6 \%)$ & $<0,001$ \\
\hline Thiazid-Diuretikum & 129 & $(37,9 \%)$ & 138 & $(41,8 \%)$ & 73 & $(41,0 \%)$ & 0,001 \\
\hline Schleifendiuretikum & 19 & $(5,6 \%)$ & 48 & $(14,5 \%)$ & 55 & $(30,9 \%)$ & $<0,001$ \\
\hline Andere Diuretika & 10 & $(2,9 \%)$ & 17 & $(5,2 \%)$ & 8 & $(4,5 \%)$ & $\begin{array}{r}\text { n. s. } \\
(0,851)\end{array}$ \\
\hline Aldosteron-Antagonist & 2 & $(0,6 \%)$ & 6 & $(1,8 \%)$ & 7 & $(3,9 \%)$ & $\begin{array}{r}\text { n.s. } \\
(0,172)\end{array}$ \\
\hline Calcium-Antagonist & 63 & $(18,5 \%)$ & 85 & $(25,8 \%)$ & 47 & $(26,4 \%)$ & $<0,001$ \\
\hline Statin & 74 & $(21,8 \%)$ & 119 & $(36,1 \%)$ & 105 & $(59,0 \%)$ & $<0,001$ \\
\hline ASA & 112 & $(32,9 \%)$ & 115 & $(34,8 \%)$ & 93 & $(52,2 \%)$ & $<0,001$ \\
\hline Vitamin-K-Antagonist & & $(3,8 \%)$ & 27 & $(8,2 \%)$ & 17 & $(9,6 \%)$ & $\begin{array}{r}\text { n.s. } \\
(0,084)\end{array}$ \\
\hline Orales Antidiabetikum & 0 & $0 \%$ & 214 & $(64,8 \%)$ & 52 & $(29,2 \%)$ & $<0,001$ \\
\hline
\end{tabular}

n.s. $=$ nicht signifikant $\sim$ Cochran-Armitage-Trendtest

Tab. 7: Medikation der Glucosestoffwechsel-Gruppen. Anzahl und (Prozentsatz).

Deutlich wird, dass Patienten umso intensiver mit kardiovaskulärer Medikation wie ACE-Hemmern, AT1-Rezeptor-Antagonisten, Betablockern, Diuretika, CalciumAntagonisten, sowie Statinen, Aminosalicylaten (ASA) und oralen Antidiabetika therapiert wurden, je schlechter ihr Glucosestoffwechsel war. Keine mit dem Grad der Glucosestoffwechsel-Störung signifikant häufigere Anwendung fanden hingegen Aldosteron-Antagonisten, andere Diuretika (nicht Thiazid- oder Schleifendiuretika) sowie Vitamin-K-Antagonisten.

\subsubsection{Metabolische Parameter}

\subsubsection{Parameter des Glucosestoffwechsels}

Bei einem Referenzbereich von 2,9 - 4,2\% ergaben die Messungen des HbA1cWertes bei Patienten mit normalem Glucosestoffwechsel einen Median von 3,7\%, bei nicht-insulinpflichtigen Diabetikern 4,9\% und bei insulinabhängigen Diabetikern einen Median von 5,3\% (vgl. Abb. 4). 


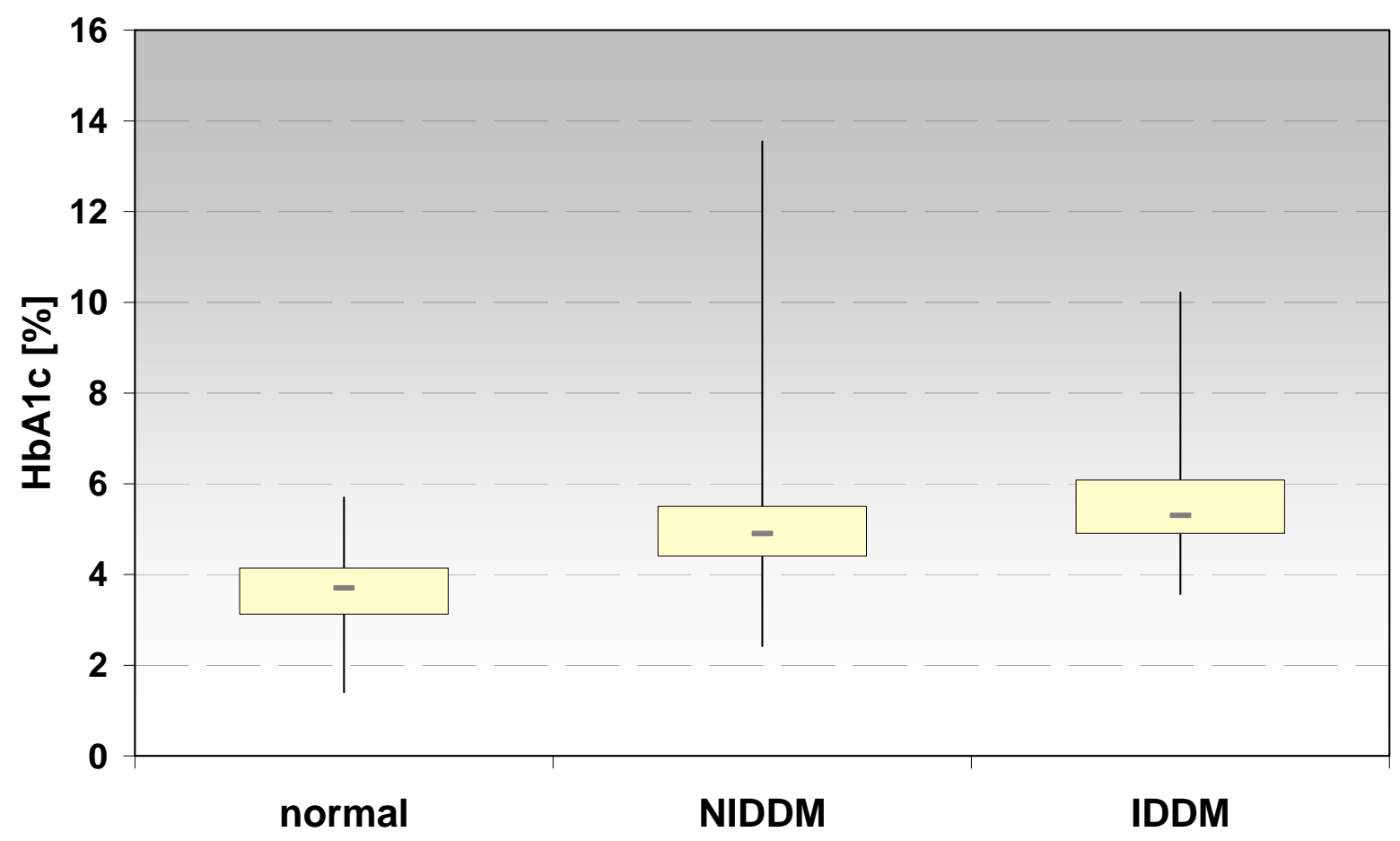

Abb. 4: HbA1c-Werte der verschiedenen Gruppen des Glucosestoffwechsels: Median (waagerechte Linie), Interquartil-Bereich (Fläche im Rechteck) sowie Min.und Max.-Werte (senkrechte Linie).

Insgesamt war also, wie zu erwarten, ein Anstieg des glycosylierten Hämoglobins über die Glucosestoffwechsel-Gruppen zu verzeichnen.

\subsubsection{Weitere metabolische Parameter}

Die folgende Tabelle (Tab. 8) zeigt die Ergebnisse bezüglich der verschiedenen Parameter des Fettstoffwechsels sowie bezüglich der Harnsäurewerte und Werte der geschätzten Glomerulären Filtrationsrate (eGFR = estimated GFR). 


\begin{tabular}{|c|c|c|c|c|c|c|c|}
\hline Variable [Einheit] & \multicolumn{2}{|c|}{ Normal } & \multicolumn{2}{|c|}{ NIDDM } & \multicolumn{2}{|c|}{ IDDM } & Test $^{\$}$ \\
\hline & \multicolumn{2}{|c|}{$N=343$} & \multicolumn{2}{|c|}{$N=335$} & \multicolumn{2}{|c|}{$N=178$} & P-Wert \\
\hline $\begin{array}{l}\text { Gesamtcholesterin } \\
{[\mathrm{mg} / \mathrm{dl}]}\end{array}$ & 206 & [179; 231] & 189 & {$[164 ; 217]$} & 181 & {$[151 ; 196]$} & $<0,0005$ \\
\hline $\begin{array}{l}\text { LDL-Cholesterin } \\
{[\mathrm{mg} / \mathrm{dl}]}\end{array}$ & 128 & {$[108 ; 151]$} & 115 & {$[99 ; 141]$} & 105 & {$[82 ; 120]$} & $<0,0005$ \\
\hline $\begin{array}{l}\text { HDL-Cholesterin } \\
{[\mathrm{mg} / \mathrm{dl}]}\end{array}$ & 55 & {$[45 ; 65]$} & 46 & {$[40 ; 56]$} & 44 & {$[37 ; 56]$} & $<0,0005$ \\
\hline $\begin{array}{l}\text { Harnsäure } \\
{[\mathrm{mmol} / /]}\end{array}$ & 5,8 & {$[4,9 ; 6,7]$} & 6,3 & {$[5,5 ; 7,2]$} & 6,1 & {$[5,2 ; 7,3]$} & $<0,0005$ \\
\hline $\begin{array}{l}\text { eGFR } \\
{\left[\mathrm{ml} / \mathrm{min} / 1.73 \mathrm{~m}^{2}\right]}\end{array}$ & 71,2 & {$[63,3 ; 78,9]$} & 71,6 & {$[63,5 ; 88,0]$} & 71,4 & {$[60,1 ; 78,5]$} & $\begin{array}{r}\text { n.s. } \\
(0,64)\end{array}$ \\
\hline
\end{tabular}

n.s. = nicht signifikant \$Jonckheere-Terpstra-Test

Tab. 8: Cholesterin-, Harnsäure-, und Glomeruläre Filtrationsrate (GFR) der Glucosestoffwechsel-Gruppen. Median und [Interquartilbereich].

Erstaunlicherweise waren Gesamtcholesterin und LDL-Cholesterin bei Diabetikern niedriger als bei Patienten mit normalem Glucosestoffwechsel. Das protektive HDLCholesterin fiel jedoch ebenfalls mit der Schwere der Glucosestoffwechsel-Störung (Tab. 8).

Harnsäurewerte waren bei Diabetikern höher, für die glomeruläre Filtrationsrate (eGFR) hingegen konnte kein signifikanter Unterschied zwischen den Gruppen ermittelt werden.

\subsection{Diastolische Dysfunktion im Kollektiv}

Bei 61 Patienten (5,6\%) konnte die diastolische Funktion des Herzens echokardiographisch nicht analysiert oder klassifiziert werden, zumeist bedingt durch das Vorliegen eines Vorhofflimmerns. Somit wurden für die folgenden Analysen der diastolischen Funktion insgesamt 1024 Patienten berücksichtigt.

\subsubsection{Prävalenz der diastolischen Dysfunktion}

Die Häufigkeit der diastolischen Dysfunktion ungeachtet des Schweregrades war signifikant höher beim Vorhandensein einer Glucosestoffwechsel-Störung $(p<0,001)$. Die folgende Abbildung (Abb. 5) verdeutlicht diese Ergebnisse: 


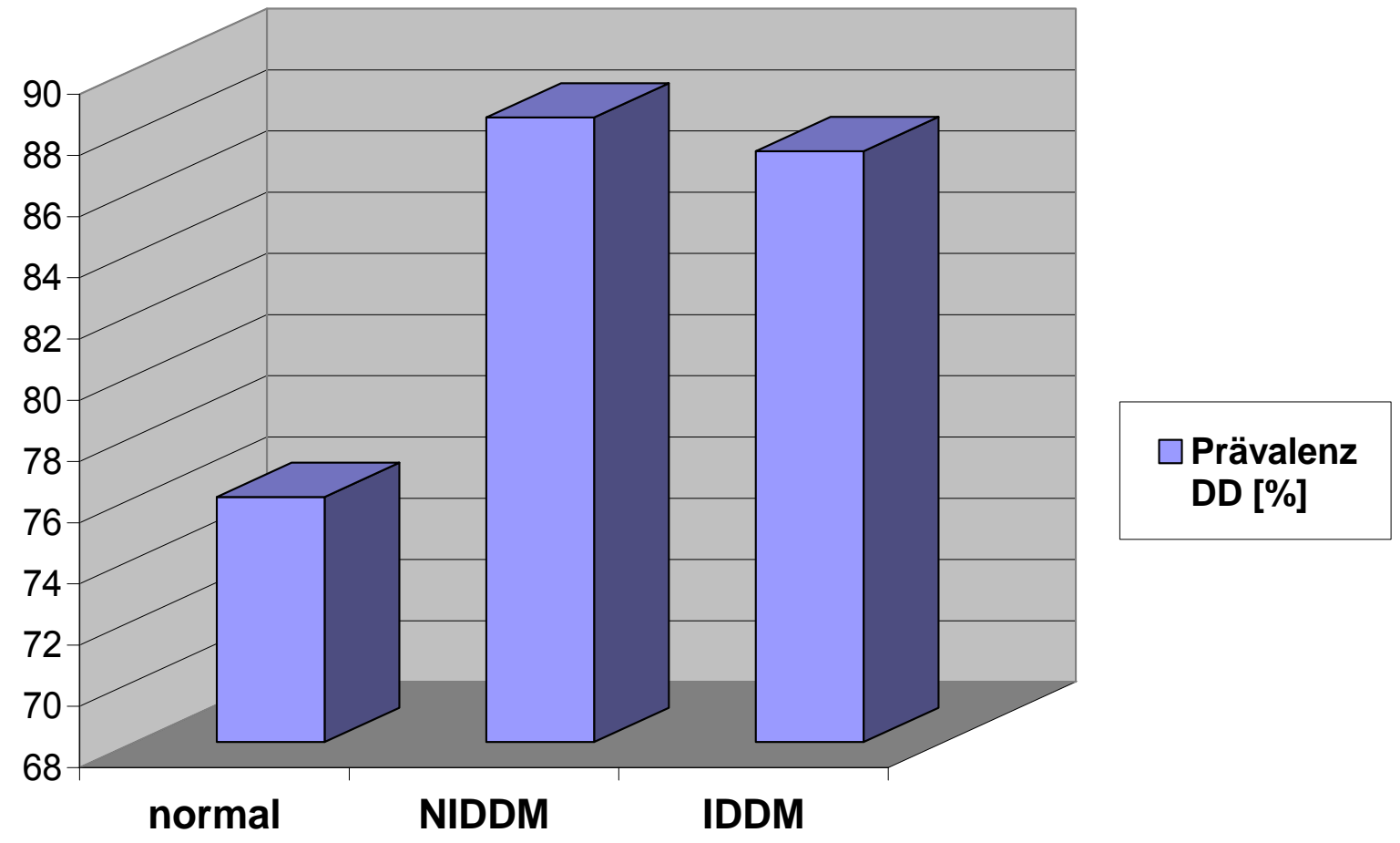

Abb. 5: Prävalenz der diastolischen Dysfunktion [\%]

Die höchste Prävalenz einer diastolischen Dysfunktion wiesen die nichtinsulinabhängigen Diabetiker mit $88,4 \%$ auf, gefolgt von den insulinabhängigen Diabetikern mit $86,7 \%$. Bei den Patienten mit normalem Glucosestoffwechsel hingegen war bei $76,0 \%$ eine diastolische Dysfunktion zu finden.

Die Verteilung der verschiedenen Schweregrade der diastolischen Dysfunktion nach den Kriterien der American Society of Echocardiology (ASE-Grade) (Quinones et al. 2002) über die einzelnen Glucosestoffwechsel-Gruppen verdeutlicht die folgende Abbildung (Abb. 6): 


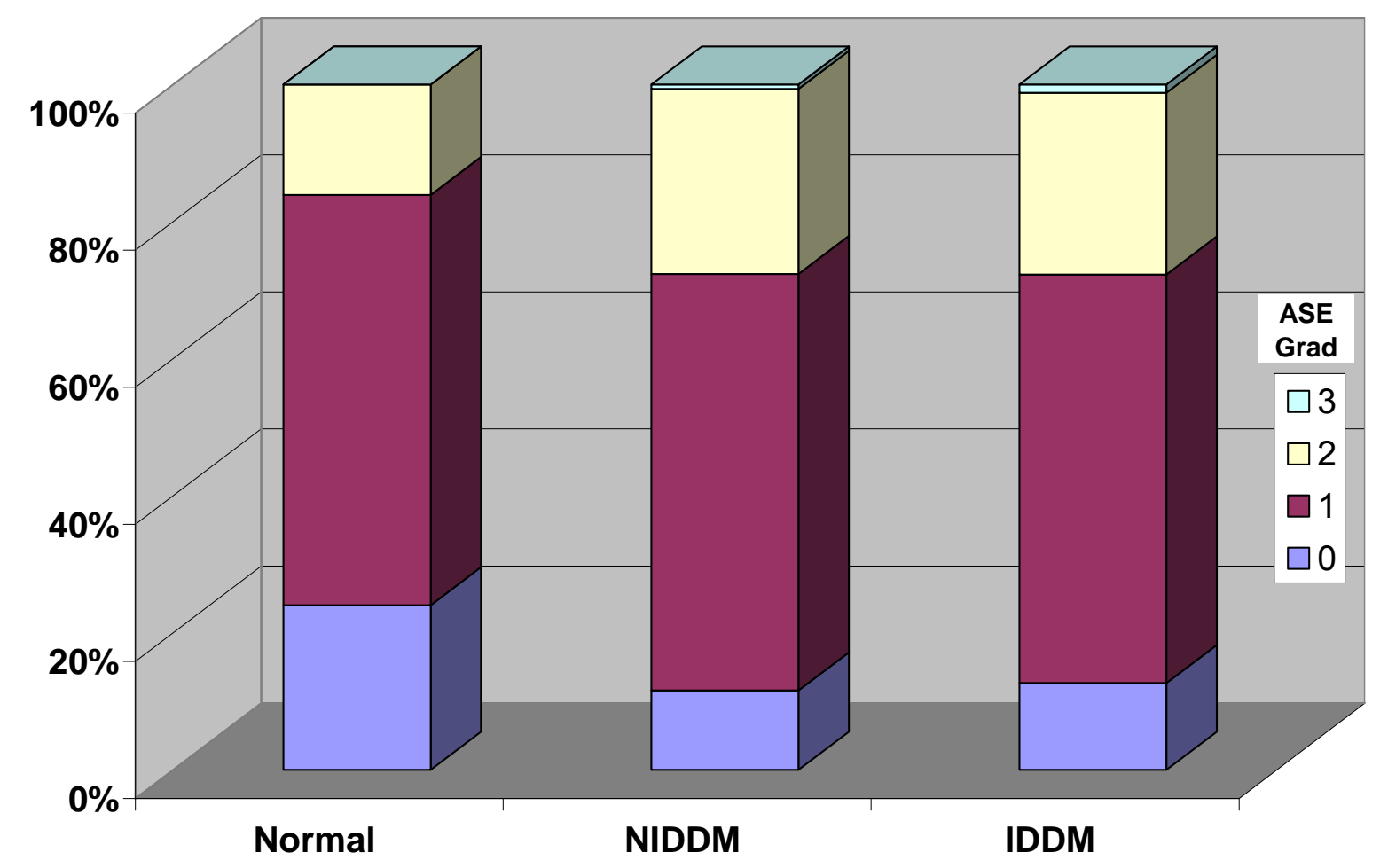

Abb. 6: Schweregrade der diastolischen Dysfunktion in den GlucosestoffwechselGruppen [\%]

Eine normale diastolische Funktion des Herzens (ASE-Grad 0) bestand bei $24 \%$ der Nicht-Diabetiker, hingegen nur bei $12 \%$ der nicht-insulinpflichtigen und bei $13 \%$ der insulinpflichtigen Diabetiker. Eine milde diastolische Dysfunktion (ASE-Grad 1) war allerdings in den Gruppen fast gleich repräsentiert: Sie fand sich bei $60 \%$ der Patienten ohne Glucosestoffwechsel-Störung, bei $60 \%$ der nicht-insulinpflichtigen und bei $59 \%$ der insulinpflichtigen Diabetiker. Eine moderate diastolische Dysfunktion (ASE-Grad 2) war bei Diabetikern häufiger (NIDDM und IDDM 27\%) als bei NichtDiabetikern (16\%). Bei keinem der Patienten mit normalem Glucosestoffwechsel konnte eine schwere diastolische Dysfunktion festgestellt werden. Dagegen wurde bei zwei nicht-insulinpflichtigen und bei zwei insulinpflichtigen Diabetikern eine schwere diastolische Dysfunktion diagnostiziert (entspricht jeweils unter $1 \%$ der Glucosestoffwechsel-Gruppe). Insgesamt zeigt sich also bei Diabetikern nicht nur eine höhere Prävalenz der diastolischen Dysfunktion, sondern auch eine Verschiebung hin zu höheren Schweregraden.

Der Schweregrad der Glucosestoffwechsel-Störung zeigte sich ebenfalls signifikant assoziiert mit der Prävalenz einer diastolischen Dysfunktion bei Durchführung einer schrittweisen logistischen Regressionsanalyse, unter Berücksichtigung folgender 
signifikant assoziierter Variablen: Herzfrequenz, systolischer und diastolischer Blutdruck, sowie aktuell bestehende oder anamnestisch bekannte Herzinsuffizienz.

\subsubsection{Echokardiographische Parameter}

Im Folgenden sollen zunächst die Unterschiede in den Gruppen hinsichtlich der für die Einteilung in die Schweregrade der diastolischen Funktion relevanten Parameter (E/A-Ratio, E/e'-Ratio und S/D-Ratio) dargestellt werden. Anschließend werden weitere Parameter, deren Veränderung hinweisend auf eine diastolische Dysfunktion sein kann, aufgeführt.

\subsubsection{ElA-Ratio}

Die folgende Tabelle (Tab. 9) stellt die Ergebnisse bezüglich E- und A-WellenGeschwindigkeit, sowie E/A-Ratio in den jeweiligen Gruppen jeweils als Median und den Interquartilbereich in eckigen Klammern dar.

\begin{tabular}{|c|c|c|c|c|c|c|c|}
\hline $\begin{array}{l}\text { Variable } \\
\text { [Einheit] }\end{array}$ & \multicolumn{2}{|c|}{ Normal } & \multicolumn{2}{|c|}{ NIDDM } & \multicolumn{2}{|c|}{ IDDM } & Test $^{\$}$ \\
\hline & \multicolumn{2}{|c|}{$N=343$} & \multicolumn{2}{|c|}{$N=335$} & \multicolumn{2}{|c|}{$N=178$} & P-Wert \\
\hline $\begin{array}{l}\text { E-Wellen } \\
\text { Geschwindigkeit } \\
{[\mathrm{cm} / \mathrm{s}]}\end{array}$ & 71 & {$[59,83]$} & 70 & {$[58,82]$} & 73 & {$[62 ; 89]$} & 0,017 \\
\hline $\begin{array}{l}\text { A-Wellen } \\
\text { Geschwindigkeit } \\
{[\mathrm{cm} / \mathrm{s}]}\end{array}$ & 77 & {$[65,89]$} & 82 & {$[70,95]$} & 83 & {$[69 ; 102]$} & $<0,0005$ \\
\hline E/A-Ratio & 0,88 & {$[0,74 ; 1,13]$} & 0,83 & {$[0,69 ; 1,05]$} & 0,83 & {$[0,70 ; 1,14]$} & 0,088 \\
\hline
\end{tabular}

$\$$ Jonckheere-Terpstra-Test

Tab. 9: E- und A-Wellen Geschwindigkeit, sowie E/A-Ratio in den Glucosestoffwechsel-Gruppen. Median und [Interquartilbereich].

Deutlich wird, dass bei allen drei Parametern ein signifikanter Unterschied zwischen den Glucosestoffwechsel-Gruppen bestand. A- und E-Wellen-Geschwindigkeit stiegen mit dem Grad der Glucosestoffwechsel-Störung an, sie waren somit bei insulinpflichtigen Diabetikern am größten (E-Welle: Median $73 \mathrm{~cm} / \mathrm{s}$; A-Welle: Median $83 \mathrm{~cm} / \mathrm{s})$.

Die E/A-Ratio war bei Diabetikern lediglich tendenziell, jedoch nicht signifikant niedriger als bei Nicht-Diabetikern $(p=0,088)$. In diesem Zusammenhang sollte jedoch der Mechanismus der Pseudonormalisation bedacht werden (s.o.), der ja 
keinen kontinuierlichen Anstieg der E/A-Ratio mit der Schwere der diastolischen Dysfunktion erwarten lässt.

\subsubsection{Ele'-Ratio}

Als funktioneller Indikator für den linksventrikulären enddiastolischen Druck wurde die E/e'-Ratio berechnet. Verglichen mit Patienten mit normalem Glucosestoffwechsel hatten Diabetiker eine signifikant höhere E/e'-Ratio $(p<0,0005)$.

\begin{tabular}{|c|c|c|c|c|c|c|c|}
\hline $\begin{array}{l}\text { Variable } \\
\text { [Einheit] }\end{array}$ & \multicolumn{2}{|c|}{ Normal } & \multicolumn{2}{|c|}{ NIDDM } & \multicolumn{2}{|c|}{ IDDM } & Test $^{\$}$ \\
\hline & \multicolumn{2}{|c|}{$N=343$} & \multicolumn{2}{|c|}{$N=335$} & \multicolumn{2}{|c|}{$N=178$} & P-Wert \\
\hline$E / e^{\prime}$ & 9,0 & {$[7,3 ; 11,2]$} & 9,5 & {$[7,4 ; 11,8]$} & 10,7 & {$[8,3 ; 13,7]$} & $<0,0005$ \\
\hline$a^{\prime}[\mathrm{cm} / \mathrm{s}]$ & 11,2 & {$[9,3 ; 13,0]$} & 11,0 & {$[9,6 ; 13,0]$} & 11,0 & {$[9,1 ; 13,1]$} & n. s. \\
\hline$e^{\prime} / a^{\prime}$ & 0,70 & {$[0,55 ; 0,90]$} & 0,64 & {$[0,55 ; 0,85]$} & 0,63 & {$[0,47 ; 0,83]$} & 0,051 \\
\hline
\end{tabular}

n.s. = nicht signifikant \$Jonckheere-Terpstra-Test

Tab. 10: Mitralringspitzengeschwindigkeiten. Median und [Interquartilbereich].

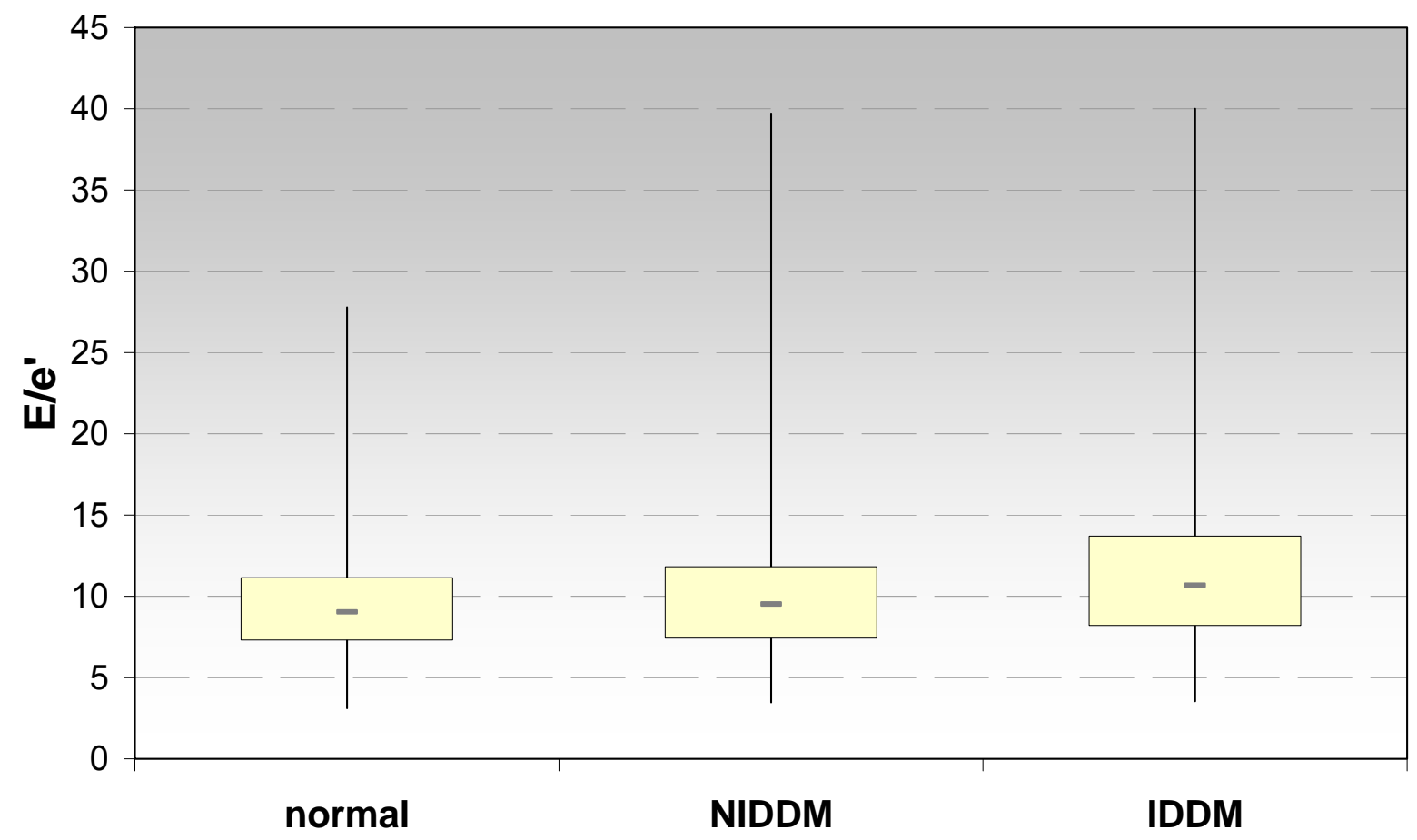

Abb. 7: E/e'-Ratio: Median und Interquartilbereich der Gruppen.

Auch wiesen insulinpflichtige Diabetikern verglichen mit nicht-insulinpflichtigen Diabetikern signifikant höhere E/e'-Werte auf $(p<0,05)$. 
Die Mitralringspitzengeschwindigkeiten alleine ergaben für e' einen signifikanten Abfall der Werte über die Gruppen, nicht jedoch für die e'/a'-Ratio, als Möglichkeit der Demaskierung der oben genannten Pseudonormalisation. Auch für a' konnte kein signifikanter Unterschied nachgewiesen werden (vgl. Tab. 10).

\subsubsection{S/D-Ratio}

Eine Verringerung der S/D-Ratio auf Werte <1 spricht für eine diastolische Dysfunktion. Es konnte zwar ein geringer Abfall des Medians der Ratio über die Gruppen festgestellt werden, die Unterschiede zwischen den Gruppen erwiesen sich jedoch als nicht signifikant (vgl. Tab. 11).

\begin{tabular}{|c|c|c|c|c|c|c|c|}
\hline Variable [Einheit] & \multicolumn{2}{|c|}{ Normal } & \multicolumn{2}{|c|}{ NIDDM } & \multicolumn{2}{|c|}{ IDDM } & Test $^{\$}$ \\
\hline & \multicolumn{2}{|c|}{$N=343$} & \multicolumn{2}{|c|}{$\mathrm{N}=335$} & \multicolumn{2}{|c|}{$N=178$} & $\mathbf{P}$ \\
\hline S/D-Ratio & 1,41 & {$[1,21 ; 1,66]$} & 1,40 & {$[1,18 ; 1,67]$} & 1,38 & {$[1,12 ; 1,61]$} & n. s. \\
\hline $\mathrm{PVSF}[\mathrm{cm} / \mathrm{s}]$ & 63 & {$[54 ; 73]$} & 61 & {$[54 ; 69]$} & 60 & {$[51 ; 70]$} & 0,03 \\
\hline PVDF [cm/s] & 44 & {$[37 ; 52]$} & 43 & {$[37 ; 51]$} & 45 & {$[37 ; 54]$} & n. s. \\
\hline PVAF $[\mathrm{cm} / \mathrm{s}]$ & 30 & [27; 33] & 30 & [26; 33] & 29 & [26; 32] & n. s. \\
\hline
\end{tabular}

Tab. 11: Pulmonalvenenflussprofil in den Gruppen. Median und [Interquartilbereich].

Lediglich für den systolischen Pulmonalvenenfluss (PVSF) konnte ein signifikanter Abfall $(p=0,003)$ über die Gruppen nachgewiesen werden. Bezüglich des diastolischen Pulmonalvenenflusses (PVDF) und atrialen Rückflusses (PVAF) bestanden jedoch keine signifikanten Differenzen.

\subsubsection{Weitere echokardiographische Parameter der diastolischen Funktion}

Als Zeichen einer Hypertrophie des linken Ventrikels können enddiastolische linksventrikuläre Septumdicke (IVS) und enddiastolische Hinterwanddicke (LVPW) erhöht sein. Ferner gehen diese Parameter wie oben dargestellt in die Berechnung der linksventrikulären Masse (LVM) gemäß der Formel nach Devereux ein. Die folgende Tabelle (Tab. 12) zeigt diesbezügliche Ergebnisse für die verschiedenen Glucosestoffwechsel-Gruppen. 


\begin{tabular}{|l|rr|rr|rr|r|r|}
\hline $\begin{array}{l}\text { Variable } \\
\text { [Einheit] }\end{array}$ & \multicolumn{2}{|c|}{ Normal } & \multicolumn{2}{|c|}{ NIDDM } & \multicolumn{2}{c|}{ IDDM } & Test $^{\$}$ \\
\hline & \multicolumn{2}{|c|}{$\mathbf{N}=\mathbf{3 4 3}$} & \multicolumn{2}{c|}{$\mathbf{N}=\mathbf{3 3 5}$} & \multicolumn{2}{c|}{$\mathbf{N = 1 7 8}$} & P-Wert \\
\hline IVS [mm] & 12 & {$[11,13]$} & 13 & {$[11 ; 14]$} & 13 & {$[11 ; 14]$} & 0,003 \\
\hline LVPW [mm] & 11 & {$[10,12]$} & 12 & {$[11 ; 13]$} & 12 & {$[11 ; 13]$} & 0,0005 \\
\hline LVMI [g/m²] & 113,9 & {$[97,8 ; 130,9]$} & 121,2 & {$[102,0 ; 141,6]$} & 120,6 & {$[100,2 ; 140,0]$} & 0,033 \\
\hline
\end{tabular}

$\$$ Jonckheere-Terpstra-Test

Tab. 12: Parameter der linksventrikulären Masse. Median und [Interquartilbereich].

Deutlich wird, dass sowohl die linksventrikuläre enddiastolische interventrikuläre Septumdicke (IVS) als auch die linksventrikuläre enddiastolische Hinterwanddicke (LVPW) bei Diabetikern größer waren als bei Patienten mit normalem Glucosestoffwechsel. Auch der Linksventrikuläre Massenindex (LVMI) als Hinweis auf eine linksventrikuläre Hypertrophie und eine demzufolge eingeschränkte diastolische Funktion war bei Diabetikern (NIDDM und IDDM) signifikant höher als bei Nicht-Diabetikern.

Eine Vergrößerung des linksatrialen Durchmessers (LAD) ist ebenfalls ein Anzeichen für eine diastolische Dysfunktion. Auch dieser war bei den Diabetikern signifikant größer $(p<0.0005)$ als bei Patienten ohne Glucosestoffwechsel-Störung (vgl. Abb. 8). 


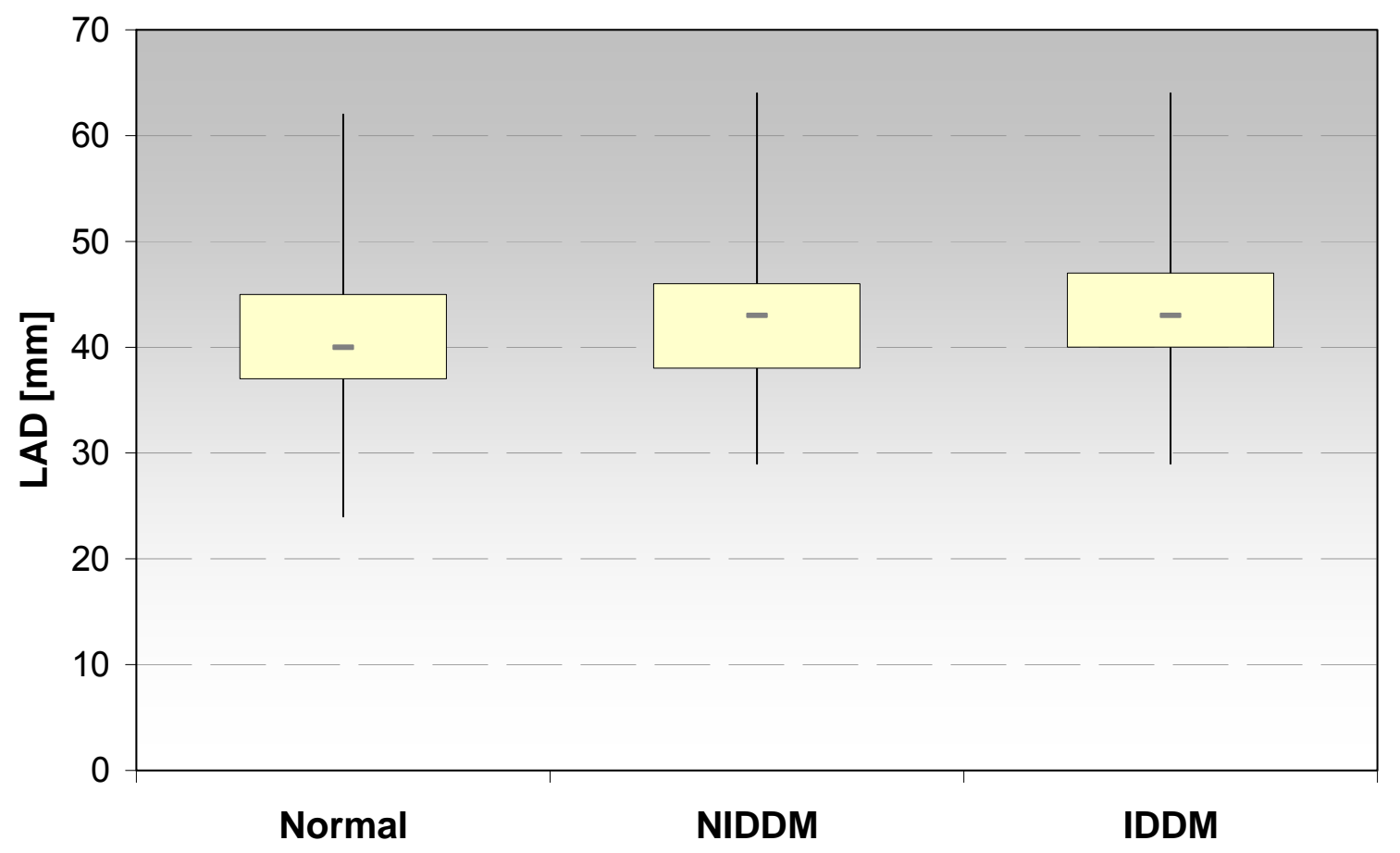

Abb. 8: Linksatrialer Durchmesser [mm] in den Gruppen. Median, Interquartilbereich, Min.- und Max.-Werte.

Keine signifikanten Unterschiede zwischen den Gruppen konnten für die E-WellenDezelerationszeit (EDCT) und die isovolumetrische Relaxationszeit (IVRT) festgestellt werden (Tab. 13).

\begin{tabular}{|c|c|c|c|c|c|c|c|}
\hline Variable [Einheit] & \multicolumn{2}{|c|}{ Normal } & \multicolumn{2}{|c|}{ NIDDM } & \multicolumn{2}{|c|}{ IDDM } & Test $^{\$}$ \\
\hline & \multicolumn{2}{|c|}{$N=343$} & \multicolumn{2}{|c|}{$N=335$} & \multicolumn{2}{|c|}{$N=178$} & $\mathbf{P}$ \\
\hline EDcT [ms] & 240 & {$[202,298]$} & 256 & {$[201,305]$} & 244 & {$[195,300]$} & n. s. \\
\hline IVRT [ms] & 100 & {$[90,119]$} & 105 & {$[90,120]$} & 97 & {$[85,115]$} & n. s. \\
\hline
\end{tabular}

n.s. = nicht signifikant $\$$ Jonckheere-Terpstra-Test

Tab. 13: E-Wellen-Dezelerationszeit (EDcT), isovolumetrische Relaxationszeit (IVRT) und Propagationsgeschwindigkeit. Median und [Interquartilbereich].

Allerdings ist die IVRT wie oben dargestellt vorlast- und frequenzabhängig und kann bei erhöhten Drücken des linken Vorhofes wieder im Normalbereich liegen. Die EWellen-Dezelerationszeit unterliegt wie das E/A-Verhältnis dem Mechanismus der Pseudonormalisation. 


\subsubsection{Korrelation von Glucosehomöostase und echokardiographischen Parametern}

\subsubsection{Korrelation des HbA1c mit E/e'}

In der bivariaten Analyse korrelierte E/e' signifikant mit dem HbA1c-Wert ( $r=0,2$; $p<0,001)$. Sogar bei alleiniger Betrachtung der Patienten mit HbA1c-Werten $<5 \%$ bestand eine schwache Assoziation zwischen E/e' und $\mathrm{HbA1c}(r=0,14 ; p<0,01)$.

Bei insulinabhängigen Diabetikern korrelierte $E / e^{\prime}$ signifikant mit der täglichen Insulindosis $(p<0,05, r=0,19)$, nicht jedoch mit der Dauer des Diabetes $(p=0,08, r$ $0,11)$.

Auch bei der multivariaten linearen Analyse, bei der der Einfluss von Geschlecht, koronarer Herzkrankheit (KHK), Alter, Body Mass Index (BMI), Herzfrequenz, systolischem und diastolischem Blutdruck, linksventrikulärem Massenindex (LVMI) und linksatrialem Durchmesser (LAD) auf E/e' herausgerechnet wurde, blieb eine signifikante Assoziation zwischen HbA1c und E/e' bestehen ( $p=0,004)$. Geschlecht, Alter, Herzfrequenz und linksatrialer Durchmesser waren auch in der schrittweisen logistischen Regressionsanalyse Variablen mit signifikantem Einfluss auf E/e'.

Wurde an Stelle des HbA1c der Status des Glucosemetabolismus verwendet (normal, NIDDM, IDDM), so ergab sich auch für diesen eine signifikante Assoziation mit E/e'.

\subsubsection{Korrelation von Stoffwechselparametern mit LVMI und LAD}

Linksventrikulärer Massenindex (LVMI) und linksatrialer Durchmesser (LAD) als strukturelle Marker der diastolischen Funktion korrelierten signifikant mit dem HbA1c (jeweils $\mathrm{p}$ <0.05). Die multivariaten Analyse ergab jedoch keine signifikante Assoziation zwischen LVMI, LAD und dem HbA1c-Wert.

Außerdem bestand eine hochsignifikante negative Korrelation zwischen HDLCholesterin und LAD ( $r=-0.262)$ sowie LVMI $(r=-0.329)$. Bei Patienten mit einem $\mathrm{HbA1c} \leq 5 \%$ korrelierten LAD und LVMI jedoch nicht mit dem HbA1c.

Weder zur Dauer des Diabetes mellitus noch zur täglichen Insulindosis der IDDMGruppe gab es eine Korrelation von LVMI oder LAD. 


\subsubsection{Der 6-Minuten-Gehtest : Glucosehomöostase, diastolische Dysfunktion und körperliche Leistungsfähigkeit}

Der 6-Minuten-Gehtest stellt einen einfachen Indikator für die jeweilige physische Belastungsfähigkeit dar. In den untersuchten Patientengruppen zeigte sich eine deutliche Verschlechterung der Leistung von Nicht-Diabetikern (Median 550m), über nicht-insulinpflichtige Diabetiker (Median 506m) hin zu den insulinabhängigen Diabetikern mit einem Median von 470m ( $p<0,0005)$.

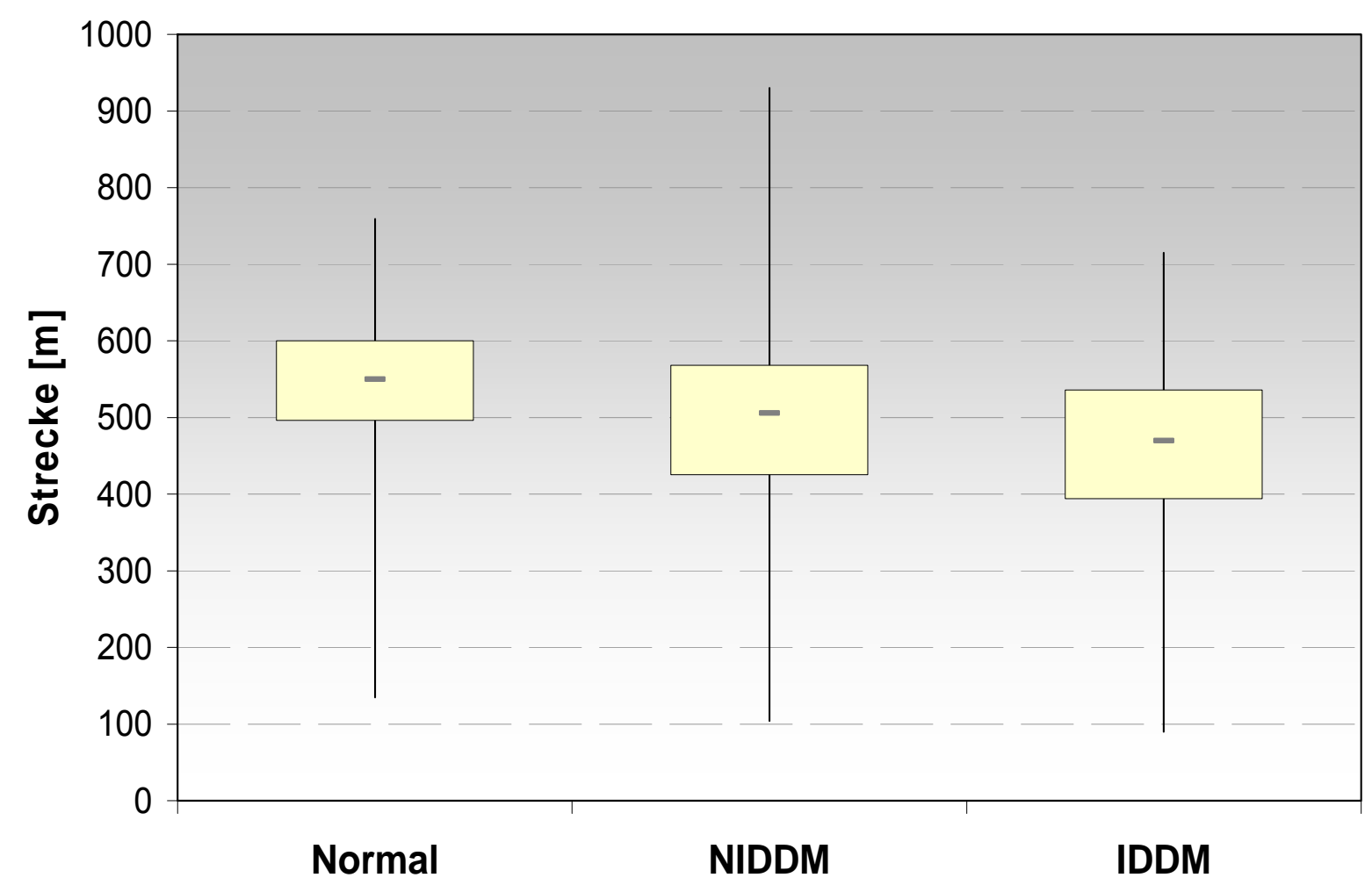

Abb. 9: 6-Minuten-Gehstrecke in den Glucosestoffwechsel-Gruppen. Median, Interquartilbereich, Min.- und Max.-Werte.

Die Schweregrade ,Moderate diastolische Dysfunktion' und ,Schwere diastolische Dysfunktion' wurden aufgrund der geringen Fallzahlen zu einer Gruppe zusammengefasst. Diese sowie die Gruppe ,Milde diastolische Dysfunktion' korrelierten signifikant mit der 6-Minuten-Gehstrecke $(r=-0,29 ; p<0,001)$.

Auch E/e'-Ratio und 6-Minuten-Gehstrecke korrelierten signifikant miteinander ( $r=-$ $0,17 ; p<0,001)$. Zwischen Gehstrecke und Ejektionsfraktion hingegen konnte lediglich eine schwache Korrelation gefunden werden ( $r=0,09 ; p<0,01)$. 


\subsubsection{Geschlechtsspezifische Unterschiede der diastolischen Funktion}

E/e' war bei Frauen signifikant höher als bei Männern. Der Median betrug bei Frauen 10,63, bei Männern hingegen 9,75 ( $p<0,05)$. Für den $\mathrm{HbA} 1 \mathrm{c}$ ließ sich kein geschlechtsspezifischer Unterschied feststellen. Die Prävalenz der Herzinsuffizienz unterschied sich zwischen Männern und Frauen nicht, bezüglich einer koronaren Herzerkrankung (KHK) wurde jedoch bei Frauen eine signifikant geringere Prävalenz nachgewiesen.

\subsection{Echokardiographische Parameter der systolischen Ventrikelfunktion}

Anhand der folgenden Tabelle (Tab. 14) können die Unterschiede in den Gruppen bezüglich echokardiographischer Parameter der systolischen Ventrikelfunktion nachvollzogen werden. Dies sind im Einzelnen: Linksventrikulärer enddiastolischer und endsystolischer Durchmesser (LVEDD und LVESD), linksventrikuläres enddiastolisches und endsystolisches Volumen (LVEDV und LVESV) sowie linksventrikuläre Ejektionsfraktion (LVEF).

\begin{tabular}{|c|c|c|c|c|c|c|c|}
\hline Variable [Einheit] & \multicolumn{2}{|c|}{ Normal } & \multicolumn{2}{|c|}{ NIDDM } & \multicolumn{2}{|c|}{ IDDM } & Test $^{\$}$ \\
\hline & \multicolumn{2}{|c|}{$N=343$} & \multicolumn{2}{|c|}{$N=335$} & \multicolumn{2}{|c|}{$N=178$} & P-Wert \\
\hline LVEDD [mm] & 49 & {$[45,52]$} & 51 & {$[46,53$} & 51 & {$[46,54]$} & 0,011 \\
\hline LVESD [mm] & 30 & {$[26,34]$} & 31 & {$[27,36]$} & 32 & {$[28,37]$} & $<0.0005$ \\
\hline LVEDV [ml] & 88,0 & {$[71,106]$} & 94,0 & {$[76,116]$} & 90,0 & {$[74,110]$} & n. s. \\
\hline LVESV [ml] & 35,0 & {$[27,43]$} & 37,0 & {$[28,50]$} & 36,0 & {$[28,49]$} & 0,06 \\
\hline LVEF [\%] & 60 & {$[55,65]$} & 60 & {$[55,65$} & 60 & {$[54,64]$} & n. s. \\
\hline
\end{tabular}

$\$$ Jonckheere-Terpstra-Test

Tab. 14: Linksventrikuläre Parameter der systolischen Pumpfunktion. Median und [Interquartilbereich].

Sowohl enddiastolische als auch endsystolische Durchmesser waren bei Diabetikern signifikant größer als bei Patienten mit normalem Glucosestoffwechsel. Für die daraus zu errechnende prozentuale Durchmesserverkürzung (Fractional Shortening) 
als Maß für die systolische Funktion des linken Ventrikels nach der Formel:

FS [\%] $=[($ LVED-LVES)/(LVED) $] \times 100$ ergeben sich daraus jedoch keine schlechteren Werte für die Diabetiker verglichen mit Nicht-Diabetikern.

Weder für endsystolische noch für enddiastolische Volumina, aus denen sich die Ejektionsfraktion (EF) errechnet (s.o.), wurde ein signifikanter Unterschied zwischen den Gruppen gefunden. Auch der Median der Ejektionsfraktion war mit je $60 \%$ in allen drei Glucosestoffwechsel-Gruppen gleich.

Somit ergab sich also insgesamt in Bezug auf die systolische Funktion des Ventrikels kein wesentlicher Unterschied zwischen den Gruppen der GlucosestoffwechselStörung. 


\section{Diskussion}

Schon frühere Studien ergaben, dass bei einem Diabetes mellitus eine diastolische Dysfunktion häufiger besteht als in der Allgemeinbevölkerung (Zabalgoitia et al. 2000; Poirier et al. 2001). Dies konnte in dieser Arbeit bestätigt werden. Ferner wurde gezeigt, dass sich mit dem Schweregrad der Glucosestoffwechsel-Störung einzelne echokardiographische Parameter der diastolischen Ventrikelfunktion verschlechterten.

Diabetiker hatten nicht nur häufiger eine diastolische Dysfunktion, sondern waren im Vergleich zu Patienten mit normalem Glucosestoffwechsel auch schwerer betroffen. Dies ist insofern von besonderer Bedeutung, als ein höherer Schweregrad der diastolischen Dysfunktion mit einer erhöhten Mortalität einhergeht (Redfield et al. 2003; From et al. 2009).

Echokardiographische Zeichen einer diastolischen Dysfunktion können ein erster Hinweis auf die Entwicklung einer diabetischen Kardiomyopathie sein (Raev 1994; Bell 1995). Diese kann letztendlich über das sich noch asymptomatisch präsentierende Stadium der diastolischen Dysfunktion zu einer diastolischen Herzinsuffizienz mit den typischen Symptomen wie Dyspnoe, Ödemen und pulmonalem Hochdruck führen (Khavandi et al. 2009). Wünschenswert wäre eine frühe Erkennung der diastolischen Funktionsstörung bei Diabetikern noch im asymptomatischen Stadium der Erkrankung, um durch rechtzeitige Therapie ein Fortschreiten der Erkrankung zu verhindern, bzw. zu verzögern. Ob dies zum Beispiel im Rahmen eines Screenings flächendeckend möglich wäre, ist jedoch aufgrund des hohen Kosten- und Organisationsaufwandes fraglich. Andererseits müssen aber auch Kosten, welche durch die Folgen einer spät diagnostizierten diastolischen Dysfunktion entstehen, in Betracht gezogen werden. In den USA machen die Ausgaben für diastolische Herzinsuffizienz schon jetzt mehr als $25 \%$ der Gesamtkosten chronischer Herzinsuffizienz aus (Vasan und Levy 2000). Hinzu kommt, dass die Zahl der Patienten mit diastolischer Dysfunktion, bzw. Herzinsuffizienz in den westlichen Ländern in den letzten Jahren immer weiter anstieg und vermutlich weiterhin ansteigen wird (Owan et al. 2006), ebenso wie die Zahl der Diabetiker (Schwarz et al. 2007).

Im Folgenden sollen nun die Teilergebnisse dieser Arbeit diskutiert und in den aktuellen Wissensstand der Forschung eingeordnet werden. 


\subsection{Patientenkollektiv}

Für die vorliegende Studie wurden Daten von 1085 Patienten untersucht.

Bei diesem Kollektiv handelte es sich um eine Risikogruppe. Es wurden nur Patienten mit einem Risikofaktor für diastolische Dysfunktion oder mit einer Herzinsuffizienz eingeschlossen. Zudem betrug das Durchschnittsalter der Patienten 65 Jahre. Somit ergab sich ein Patientenkollektiv, welches nicht repräsentativ für die Allgemeinbevölkerung, jedoch zum Beispiel mit Patientengruppen in hausärztlichen Praxen vergleichbar sein dürfte.

Betrachtet man jedoch ausschließlich die Diabetesgruppe, so entspricht das Durchschnittalter der Diabetiker des Kollektivs (66 Jahre) in etwa dem durchschnittlichen Alter eines Typ-2-Diabetikers. Die CODE-2-Studie, welche 809 Diabetespatienten retrospektiv untersuchte, ergab ein Durchschnittsalter des deutschen Typ-2-Diabetikers von 67 Jahren (Liebl et al. 2001). Wie in der hier untersuchten DIAST-CHF-Studie waren unter den Diabetikern in der CODE-2-Studie Männer und Frauen etwa gleich repräsentiert.

Die Prävalenz des Prädiabetes lag im hier untersuchten Patientenkollektiv bei 21,1\%. Studien an großen Populationen in Europa und den USA ergaben eine etwas geringere Prävalenz von 11 bis 15\% (Saydah et al. 2001; Smith et al. 2002). Dies erklärt sich insofern, als diese Studien ältere diagnostische Kriterien der ADA und der WHO aus den Jahren 1998 und 1999 verwendeten (American Diabetes Association 1998; World Health Organization 1999). Bei diesen liegt der untere Grenzwert für eine gestörte Nüchternglucose bei 110 mg/dl. Im Jahre 2003 wurde der untere Grenzwert auf $100 \mathrm{mg} / \mathrm{dl}$ gesenkt und wird auch in den neueren, hier verwendeten ADA-Kriterien aus dem Jahre 2009 empfohlen (American Diabetes Association 2009). Somit wurde in der hier betrachteten Studie durch weitere diagnostische Grenzen bei mehr Patienten ein Prädiabetes diagnostiziert als in früheren Studien.

In der DETECT-Studie, einer bundesweiten Studie an über 55.000 Hausarztpatienten in Deutschland war ein Diabetes mellitus bei $14,7 \%$ der untersuchten hausärztlichen Patienten diagnostiziert. 61,6\% der Diabetes-Patienten waren mit Diät oder oralen Antidiabetika therapiert (NIDDM), 32\% waren insulinabhängige Diabetiker (IDDM). $6,4 \%$ der Patienten waren gar nicht therapiert (Wittchen et al. 2008).

Im Patientenkollektiv unserer Studie lag die Gesamtprävalenz eines Diabetes mellitus mit 47,3\% wesentlich höher. Der hohe Anteil lässt sich durch das Studiendesign erklären, da ein Diabetes mellitus unter anderem ein 
Einschlusskriterium für die Studienteilnahme darstellte. Die Verteilung von nichtinsulinpflichtigen und insulinpflichtigen Diabetikern war jedoch der der DETECTStudie sehr ähnlich: 65,3\% der Diabetiker waren nicht insulinpflichtig, 34,7\% insulinpflichtig.

Bei 52 Patienten wurde im Rahmen des oralen Glucosetoleranztests ein Diabetes mellitus neu diagnostiziert. Dies entspricht 8,2\% der getesteten Patienten. Diese Prozentzahl ist nahezu identisch mit den Ergebnissen von Rathmann et al., der im Rahmen der KORA-Studie 1335 Probanden ohne bekannten Diabetes zwischen 55 und 74 Jahren untersuchte und bei 8,2\% einen Diabetes mellitus fand (Rathmann et al. 2003). Allerdings ist $z u$ bedenken, dass Rathmann et al. ein aus der Normalbevölkerung zufällig ausgewähltes Kollektiv untersuchten, während sich die DIAST-CHF-Studie wie oben erwähnt auf eine Risikogruppe bezieht.

\section{2 Übergewicht und Diabetes}

Zunächst ist auffällig, dass der BMI im Median bei allen GlucosestoffwechselGruppen im Bereich einer Präadipositas lag $\left(\geq 25 \mathrm{~kg} / \mathrm{m}^{2}\right)$. Vermutlich hängt dies damit zusammen, dass ein Kollektiv mit kardiovaskulären Risikofaktoren untersucht wurde. Außerdem wurden nur ältere Patienten über 50 Jahre untersucht. Beide Merkmale sind mit einem erhöhten BMI assoziiert (Gelber et al. 2008).

Bei der Gruppe mit normaler Glucosehomöostase lag der Median des BMls bei $27 \mathrm{~kg} / \mathrm{m}^{2}$. Es ist jedoch anzunehmen, dass ein Wert von $27,3 \mathrm{~kg} / \mathrm{m}^{2}$ bei älteren Erwachsenen die niedrigste Mortalitätsrate hat (Allison et al. 2002).

Im Vergleich zu Nicht-Diabetikern zeigten sich bei Diabetikern ein signifikant höheres Körpergewicht, ein größeres Taille-Hüfte-Verhältnis, sowie ein höherer BMI. Dies bestätigt die Ergebnisse anderer Studien (Devereux et al. 2000; Vazquez et al. 2007).

Im hier untersuchten Patientenkollektiv nahm zudem die Prävalenz einer Hyperlipidämie mit dem Grad der Stoffwechselstörung zu. Die PROCAM-Studie ergab eine 2- bis 3-fach höhere Prävalenz einer schweren Hyperlipidämie bei Diabetikern (Assmann und Schulte 1988). Auch im Tiermodell zeigte sich häufiger eine Hyperlipidämie bei insulinpflichtigem Diabetes mellitus, insbesondere durch Erhöhung der Triglyceride (Ohno T et al. 2000). 
Auf den ersten Blick erstaunt, dass Gesamtcholesterin und LDL-Cholesterin im untersuchten Kollektiv bei Diabetikern niedriger waren als bei Nicht-Diabetikern. Die Menge des protektiven HDL-Cholesterins verringerte sich hingegen wie zu erwarten mit der Schwere der Glucosestoffwechsel-Störung. Zu ähnlichen Ergebnissen kamen auch Haffner et al., die bei Diabetikern verglichen mit Nicht-Diabetikern erhöhte Trigylceride, jedoch niedrigere LDL- und HDL-Werte fanden (Haffner et al. 1998). Erklären lässt sich dieses Phänomen durch eine intensivere Therapie der Diabetiker mit Statinen (vgl. Tabl. 7), die einen stärkeren Effekt auf das LDL- als auf das HDLCholesterin haben.

Bedenkt man die Rolle des Übergewichts bei der Entstehung des Diabetes mellitus Typ 2, so verwundert nicht, dass hier bei Diabetikern häufiger ein Übergewicht und erhöhte Blutfettwerte gefunden wurden.

Die Tatsache, dass Typ-2-Diabetiker häufiger unter Übergewicht leiden, ist unter kardiologischen Gesichtspunkten insofern von Bedeutung, als schon ein Übergewicht alleine die Entstehung einer diastolischen Dysfunktion begünstigt (Poirier et al. 2006). Bei einem adipösen Typ-2-Diabetiker wird die diastolische Ventrikelfunktion also einerseits durch das Übergewicht und zusätzlich durch direkte Einflüsse des Diabetes mellitus selbst verschlechtert.

Nüchtern-Triglyceride wurden in der hier untersuchten Kohorte nicht bestimmt. Angesichts einer kürzlich veröffentlichten Studie, in welcher akkumulierten Triglyceriden im Myokard eine Rolle bei der Entstehung der diabetischen Kardiomyopathie zugesprochen wird, wäre eine solche Messung sicher interessant gewesen (Rijzewijk et al. 2008).

Zusätzlich zu den oben genannten Faktoren weisen Diabetiker eine höhere Prävalenz weiterer Risikofaktoren für eine diastolische Dysfunktion auf, was im Folgenden erörtert werden soll.

\subsection{Glucosehomöostase und Risikofaktoren der diastolischen Dysfunktion}

In mehreren Studien wurde eine erhöhte Prävalenz eines arteriellen Hypertonus bei Diabetes mellitus nachgewiesen (Assmann und Schulte 1988; Taylor et al. 2010). Auch bei den hier untersuchten Glucosestoffwechsel-Gruppen konnte ein signifikanter Unterschied zwischen insulinpflichtigen, nicht-insulinpflichtigen und 
Nicht-Diabetikern festgestellt werden. Auffällig ist, dass der Median des systolischen Blutdrucks mit $144 \mathrm{mmHg}$ schon bei der Gruppe mit normalem Glucosestoffwechsel relativ hoch war. Vermutlich wurden aufgrund des Studiendesigns in die Gruppe der Patienten mit normalem Glucosestoffwechsel mehr Hypertoniker eingeschlossen, als es der Prävalenz der $>60$-Jährigen der Allgemeinbevölkerung entspricht. Diese liegt bei den 55-64-Jährigen bei 55-60\% (Hense 2000). Bei Nicht-Diabetikern der hier untersuchten Kohorte fand sich hingegen bei $87,5 \%$ ein arterieller Hypertonus.

Weitere Risikofaktoren für eine diastolische Dysfunktion sind koronare Herzerkrankung (Fischer et al. 2003), periphere arterielle Verschlusskrankheit (Zanati et al. 2009) und Schlaf-Apnoe-Syndrom (Fung et al. 2002). Bei allen drei Risikofaktoren konnte im hier untersuchten Kollektiv ein signifikanter Anstieg der Prävalenz mit der Störung des Glucosestoffwechsels nachgewiesen werden. Hinzu kommt, dass sich kardiovaskuläre Risikofaktoren bei Diabetikern schwerer auswirken. So ist beispielsweise die koronare Herzerkrankung bei Diabetikern mit einer höheren Mortalitätsrate verbunden (Haffner et al. 1998). Die im untersuchten Kollektiv nachgewiesene intensivere kardiovaskuläre Medikation der Diabetiker (vgl. Tab. 7) ist somit berechtigt und sinnvoll.

Eine Herzinsuffizienz bestand bei Diabetikern des Kollektivs häufiger als bei NichtDiabetikern. Passend zu diesem Ergebnis fanden Iribarren et al. 2000 im Rahmen einer Studie an über 45.000 Diabetikern, dass jeder Anstieg des HbA1c um 1\% mit einem erhöhten Risiko für eine Herzinsuffizienz von $8 \%$ einherging (Iribarren et al. 2001).

In verschiedenen Studien war Diabetes mellitus ein Risikofaktor für Vorhofflimmern (Benjamin et al. 1995; Movahed et al. 2005; Nichols et al. 2009). Im hier untersuchten Kollektiv konnte jedoch bei Diabetikern im Vergleich zu NichtDiabetikern keine signifikant höhere Prävalenz eines Vorhofflimmerns gefunden werden. Vermutlich steht dies in Zusammenhang mit der erstaunlicherweise insgesamt eher geringen Prävalenz eines Vorhofflimmerns in allen Gruppen. Diese lag zwischen $6,9 \%$ und $9 \%$ und liegt somit zwar noch geringfügig über der Prävalenz eines Vorhofflimmerns bei über 65-Jährigen in der Normalbevölkerung von 5,9\% (Feinberg et al. 1995). In Anbetracht der im untersuchten Kollektiv häufigen Faktoren wie arterielle Hypertonie, koronare Herzkrankheit, erhöhter BMI oder Herzinsuffizienz, welche jeder für sich das Risiko für die Entwicklung eines Vorhofflimmerns erhöhen (Wolf et al. 1996), wäre eine höhere Prävalenz des 
Vorhofflimmerns zu erwarten gewesen. Zudem ist ein Vorhofflimmern eine typische Folge der im Kollektiv häufig gefundenen diastolischen Dysfunktion (Tsang et al. 2002). Zum einen ließe sich die niedrige Prävalenz durch die Art der Datenerhebung erklären, da mittels EKG und Anamnese möglicherweise ein paroxysmales Vorhofflimmern nicht erfasst wurde, zum anderen wurde ein sich gegebenenfalls im späteren Verlauf entwickelndes Vorhofflimmern, welches nach dem Beobachtungszeitraum auftreten könnte, nicht mehr erfasst.

\subsection{Prävalenz der diastolischen Dysfunktion bei Nicht- Diabetikern}

Schon bei Patienten ohne Glucosestoffwechsel-Störung zeigte sich im Kollektiv eine vergleichsweise hohe Prävalenz der diastolischen Dysfunktion von $76 \%$. Fischer et al. fanden 2003 lediglich bei 11,1\% der Normalbevölkerung und 15,8\% der über 65Jährigen eine diastolische Funktionsstörung. Aufgrund von bedeutenden Entwicklungen der Echokardiographie innerhalb der letzten 10 Jahre stehen heute jedoch weitaus bessere Möglichkeiten zur Verfügung, eine diastolische Dysfunktion im präklinischen Stadium zu erkennen. Neue Techniken wie der Gewebedoppler (TDI) ermöglichen eine Messung der Mitralringspitzengeschwindigkeiten (e' und a') und liefern somit zusätzlich zu klassischem M-Mode, 2-D-Echokardiographie und pwDoppler wertvolle Information bezüglich der diastolischen Funktion des Herzens. Die Echokardiographie hat somit als diagnostisches Mittel an Sensitivität gewonnen.

Neuere Studien, bei denen diese verbesserten echokardiographischen Messmethoden zur Verfügung standen, kommen folglich auf eine höhere Prävalenz der diastolischen Dysfunktion von $27 \%$ in der Allgemeinbevölkerung (Kuznetsova et al. 2009) beziehungsweise $35 \%$ bei $>60$-Jährigen (Abhayaratna et al. 2006).

Dennoch ist die Prävalenz im hier untersuchten Kollektiv noch immer doppelt so hoch wie in den genannten aktuelleren Studien. Hier ist zum einen das Patientenkollektiv zu berücksichtigen, welches wie oben erläutert einer kardiovaskulären Risikogruppe entspricht. Studien an Patienten mit ähnlichen kardiovaskulären Risikofaktoren kommen auf eine fast eben so hohe Prävalenz von 55 bis $60 \%$ (Yamamoto et al. 2000; Yamada et al. 2002). Mit den oben genannten verbesserten echokardiographischen Techniken der heutigen Zeit wäre in diesen Studien wahrscheinlich eine noch etwas höhere Prävalenz gefunden worden. Saraiva et al. 
zeigten, dass mittels Gewebedoppler bei asymptomatischen Diabetikern ohne kardiale Erkrankungen bei 26\% eine diastolische Dysfunktion diagnostiziert werden konnte, während dies mittels klassischer Kriterien ohne TDI nur in 40\% dieser Fälle möglich war (Saraiva et al. 2005).

Hinzu kommt, dass in der hier vorliegenden Studie sehr sensitive Kriterien zur Klassifikation einer diastolischen Dysfunktion verwendet wurden.

Zusammenfassend lässt sich die hohe Prävalenz der diastolischen Dysfunktion im Patientenkollektiv also mit verbesserten echokardiographischen Techniken, der Selektion einer Risikogruppe und der Anwendung relativ sensitiver Diagnosekriterien begründen.

\subsection{Diastolische Dysfunktion bei Diabetikern}

\subsubsection{Diabetische Kardiomyopathie}

Eine diastolische Dysfunktion bestand wie schon in früheren Studien bei Diabetikern häufiger als bei Nicht-Diabetikern (Astorri et al. 1997; Boyer et al. 2004). Ursache ist offenbar die diabetische Kardiomyopathie, die bei Diabetikern unabhängig von Hypertonus oder koronarer Herzerkrankung zu finden ist (Bell 1995). Rubler et al. beschrieben die Erkrankung 1972 erstmals als eigene Entität. Bei der Entstehung einer diabetischen Kardiomyopathie spielt vermutlich eine Vielzahl metabolischer Störungen eine Rolle. Hyperglykämie, Hyperlipidämie und Hyperinsulinämie sowie Veränderungen im myokardialen Metabolismus führen über noch nicht vollständig geklärte Mechanismen zu strukturellen und funktionellen Störungen im Myokard von Diabetikern. Als ursächliche Mechanismen auf zellulärer Ebene werden unter anderem eine Erhöhung des oxidativen Stresses, interstitielle Fibrose, Apoptose von Myozyten, sowie Störungen des lonenhaushalts diskutiert (Factor et al. 1980; Factor et al. 1981; Kawaguchi et al. 1997).

Klinisch findet man als Ausdruck einer diabetischen Kardiomyopathie unter anderem einen gestörten myokardialen Energiestoffwechsel (Diamant et al. 2003) und eine übermäßige Aktivierung des Renin-Angiotensin-Aldosteron-Systems (Fukui et al. 2009).

Die diabetische Kardiomyopathie ist vermutlich auch der Grund dafür, dass im vorliegenden Kollektiv Diabetiker nicht nur häufiger sondern auch schwerer von einer diastolischen Dysfunktion betroffen waren. Besonders ein ASE-Grad 2 war bei 
Diabetikern wesentlich häufiger als bei den Gruppen mit normaler Glucosehomöostase, während eine normale diastolische Funktion wesentlich seltener vorhanden war.

Im untersuchten Kollektiv wiesen Diabetiker im Vergleich zur Kontrollgruppe keine schlechtere systolische Ventrikelfunktion auf. Dies bestätigt Untersuchungen, die zeigten, dass eine diabetische Kardiomyopathie primär eine Verschlechterung der diastolischen Funktion bewirkt (Raev 1994; Schannwell et al. 2002).

\subsubsection{Echokardiographische Parameter}

Bei einzelnen echokardiographischen Parametern, die auf eine diastolische Dysfunktion hindeuten, wiesen Diabetiker deutlich schlechtere Werte auf. Insbesondere E/e'-Ratio und linksatrialer Durchmesser (LAD) als Parameter für erhöhte ventrikuläre Füllungsdrücke sowie der linksventrikuläre Massenindex (LVMI) zeigten eine Verschlechterung mit dem Grad der Glucosestoffwechsel-Störung.

Eine kürzlich durchgeführte retrospektive Studie an Diabetes-Patienten in Olmsted County verdeutlicht die prognostische Bedeutung einer erhöhten E/e'-Ratio. Ein E/e'Wert von $>15$ resultierte in einem 11\%igen Anstieg der Gesamtmortalität (From et al. 2009). Auch die Wahrscheinlichkeit, eine Herzinsuffizienz zu entwickeln steigt mit der E/e'-Ratio (From et al. 2010).

In der Framingham-Studie war eine Assoziation des Diabetes mit einem erhöhten LVMI nur bei Frauen, nicht jedoch bei Männern zu finden (Galderisi et al. 1991). Andere Untersuchungen ergaben jedoch, wie auch in der hier untersuchten Kohorte, eine Assoziation bei beiden Geschlechtern (Grossman et al. 1992; Palmieri et al. 2001). Palmieri et al. fanden einen Zusammenhang der linksventrikulären Masse mit der Dauer des Diabetes sowie mit der Dauer einer arteriellen Hypertonie. Auch der linksventrikulären Masse kommt ein prognostischer Wert zu: Sie ist mit einer höheren Inzidenz kardiovaskulärer Ereignisse, einschließlich der Häufigkeit von Todesfällen, assoziiert (Levy et al. 1990).

Ähnliches gilt für den linksatrialen Durchmesser. Laut Strong-Heart-Studie ist er ein unabhängiger Risikofaktor für erste kardiovaskuläre Ereignisse (Kizer et al. 2006).

Für E/e'-Ratio, LAD und LVMI ergab sich jeweils eine Korrelation mit Parametern des Glucosestoffwechsels. Die stärkste Assoziation bestand zwischen der E/e'-Ratio und dem HbA1c-Wert. Dies ist insofern erklärbar, als der HbA1c-Wert ein guter Parameter der Langzeitblutzuckereinstellung und somit weniger anfällig für 
Schwankungen im Blutzuckerspiegel während der Testung ist. Wie oben erläutert ist dies einer der Gründe, die die American Diabetes Association in ihrer Empfehlung des $\mathrm{HbA} 1 \mathrm{c}-$ Wertes als primären diagnostischen Marker für einen Diabetes mellitus anführt (International Expert Committee 2009).

Schon bei HbA1c-Werten < $5 \%$ konnten wir eine schwache Assoziation zwischen E/e' und $\mathrm{HbA} 1 \mathrm{c}$ finden. Miyazato et al. zeigten, dass auch bei hypertonen NichtDiabetikern unabhängig von linksventrikulärer Hypertrophie schon geringe Anstiege der Plasmaglucose die diastolische Ventrikelfunktion verschlechtern (Miyazato et al. 2002).

Der negative Einfluss des Diabetes auf die diastolische Funktion ist also offenbar umso stärker je schlechter die Glucoseeinstellung. Verschiedene Mechanismen werden als Erklärung für eine Abhängigkeit von Glucoseeinstellung und diastolischer Funktion diskutiert. Diese sind sicher eng mit der Entstehung der oben erwähnten diabetischen Kardiomyopathie verknüpft.

Die bereits genannte Hyperinsulinämie führt zum einen zu einer erhöhten Insulinresistenz des peripheren Gewebes (Holmäng et al. 1996). Diese ist unabhängig von einem Diabetes mellitus ein Prädiktor für eine Herzinsuffizienz (Ingelsson et al. 2005). Zum anderen führt eine Hyperinsulinämie vermutlich durch die wachstumstimulierende Wirkung des Insulins zu einer Erhöhung der myokardialen Masse (Holmäng et al. 1996). Es resultiert ein erhöhter linksventrikulärer Massenindex (LVMI) und langfristig auch ein erhöhter linksatrialer Durchmesser (LAD) durch erhöhte Füllungsdrücke im Ventrikel. Zudem führt eine Hyperinsulinämie zu einer chronischen Aktivierung des Sympathikus mit negativen kardialen Auswirkungen (Anderson et al. 1991).

Die Hyperglykämie ihrerseits geht mit Veränderungen des myokardialen Calciumstoffwechsels und der Extrazellulärmatrix einher, was zu einer Erhöhung der ventrikulären Steifheit und verminderter Relaxation des Ventrikels führt (Poornima et al. 2006).

Zusammenfassend bewirken also chronische Hyperglykämie und Hyperinsulinämie bei Diabetikern über verschiedenste Mechanismen eine Erhöhung der ventrikulären Masse, eine Erniedrigung der Compliance des Ventrikels und erhöhte linksventrikuläre enddiastolischen Füllungsdrücke, resultierend in einer Verschlechterung der diastolischen Funktion. 


\subsubsection{Reversibilität der Diastolischen Dysfunktion beim Diabetiker}

Ob eine strikte Kontrolle und Einstellung des Blutzuckers bei Typ-2-Diabetikern die Verschlechterung der diastolischen Funktion wieder aufzuheben vermag, ist Gegenstand einer kontroversen Diskussion. Gough et al. konnten durch Verbesserung der Blutzuckereinstellung bei Diabetikern über 6 Monate keine Verbesserung der diastolischen Funktion nachweisen (Gough et al. 1995). Zu ähnlichen Ergebnissen kam auch die neuere DADD-Studie (Jarnert et al. 2009). Loimaala et al. untersuchten während eines Jahres den Effekt von körperlichem Training an 48 männlichen Typ-2-Diabetikern. Obwohl sich durch das Training die Blutzuckereinstellung verbesserte und der Blutdruck sank, war kein Effekt auf die diastolische Dysfunktion festzustellen (Loimaala et al. 2007). Sollte die diastolische Dysfunktion bei Diabetikern tatsächlich irreversibel sein, so wäre einmal mehr die Bedeutung von Prävention und Früherkennung eines gestörten Glucosemetabolismus für das Verhindern einer diastolischen Dysfunktion zu unterstreichen.

Auf der anderen Seite kamen von Bibra et al. zu dem Ergebnis, dass eine strikte Blutzucker Einstellung, insbesondere mit Insulin zu einer Verbesserung der diastolischen Funktion sowohl bei Typ-2-Diabetikern mit als auch bei Diabetikern ohne Zeichen einer ischämischen Herzerkrankung führt (von Bibra et al. 2004). Eine spätere Studie zeigte ähnliche Ergebnisse allerdings bei Typ-1-Diabetikern (Grandi et al. 2006). Im Falle einer zumindest teilweisen Reversibilität der diastolischen Funktionsstörung beim Diabetiker wäre eine striktere Blutzuckereinstellung ein möglicher therapeutischer Ansatz.

Insgesamt werden weitere Untersuchungen zeigen müssen, ob es möglich ist, mittels einer strikteren Blutzuckereinstellung eine diastolische Dysfunktion bei Diabetikern teilweise wieder aufzuheben. Hier werden insbesondere größere Studienpopulationen vonnöten sein als in den oben genannten Studien, in denen eher kleinere Patientenkollektive von circa 40 Patienten untersucht wurden.

\subsubsection{Diastolische Funktion und Dauer der Diabeteserkrankung}

$\mathrm{Da}$ sich im Verlauf der Diabeteserkrankung die Glucosehomöostase meist verschlechtert und Komplikationen wie diabetische Polyneuropathie oder Mikroalbuminurie gehäuft auftreten (Orchard et al. 1990), ist zu erwarten, dass sich auch eine diastolische Dysfunktion mit zunehmender Dauer der Erkrankung 
verschlechtert. Mehrere Untersuchungen, wie zum Beispiel die oben zitierte Studie von From et al. aus dem Jahre 2009 konnten in der Tat einen solchen Zusammenhang nachweisen. Für jedes Jahr nach der Diagnose eines Diabetes mellitus Typ 2 stieg hier die E/e'-Ratio um 0,23. In unserem Kollektiv konnte jedoch kein signifikanter Zusammenhang zwischen der Dauer des Diabetes mellitus und echokardiographischen Parametern der diastolischen Dysfunktion (E/e', LVMI, LAD) gefunden werden. Ursache könnte zum einen die Art der Datenerfassung sein. Der Zeitpunkt der Erstdiagnose des Diabetes mellitus wurde durch Aussagen der Patienten ermittelt. Dies stellt sicher eine relativ ungenaue und fehleranfällige Art der Datenerhebung dar. Zudem wurden im Vergleich zum prospektiven Studiendesigns Froms die hier verwendeten Daten retrospektiv erhoben, was zu weiteren Fehlerquellen und Ungenauigkeiten führen kann. Insgesamt sind also unsere Ergebnisse einer fehlenden Korrelation von Dauer des Diabetes und diastolischer Dysfunktion durchaus kritisch zu betrachten, zumal zahlreiche Studien zu einem gegenteiligen Ergebnis kommen (Astorri et al. 1997; Vinereanu et al. 2003; Masugata et al. 2008).

\subsubsection{NIDDM und IDDM im Vergleich}

Insulinpflichtige Diabetiker wiesen im Vergleich zu nicht-insulinpflichtigen Diabetikern eine längere Erkrankungsdauer sowie höhere $\mathrm{HbA} 1 \mathrm{c}-$ Werte auf. Aufgrund des oben erläuterten Zusammenhangs von Glucoseeinstellung und diastolischer Funktion war bei der IDDM-Gruppe im Vergleich zur NIDDM-Gruppe dementsprechend eine schlechtere diastolische Funktion zu erwarten. Bezüglich der E/e'-Ratio bestätigte sich diese Erwartung: Die E/e'-Ratio war bei insulinpflichtigen Diabetikern signifikant höher als bei nicht-insulinpflichtigen Diabetikern. Es lagen also höhere linksventrikuläre enddiastolische Füllungsdrücke vor. Bezüglich des linksatrialen Durchmessers (LAD) und des linksventrikulären Massenindexes (LVMI) bestanden jedoch keine signifikanten Unterschiede zwischen insulinpflichtigen und nichtinsulinpflichtigen Diabetikern. Die Unterschiede zwischen beiden therapeutischen Gruppen bezüglich diastolischer Funktion sind kaum erforscht. Hinzu kommt, dass im Gegensatz zu den auf Grundlage der Therapie gewählten Definitionen dieser Arbeit viele Studien einen IDDM mit einem Diabetes mellitus Typ 1 gleichsetzen, während ein NIDDM den Typ-2-Diabetes beschreibt (Astorri et al. 1997; Borgia et al. 1999). Somit war kaum ein Vergleich mit den Ergebnissen unserer Studie möglich. 
Die schlechtere E/e'-Ratio bei insulinpflichtigen Diabetikern ist am wahrscheinlichsten durch die längere Erkrankungsdauer und schlechtere Blutzuckereinstellung der IDDM Gruppe erklärbar und nicht durch einen negativen Einfluss der Insulintherapie auf das Herz, da anzunehmen ist, dass letztere sich bei Diabetikern eher positiv auf die diastolische Funktion auswirkt (von Bibra et al. 2004).

Insgesamt lässt sich also sagen, dass in der hier untersuchten Kohorte bei insulinpflichtigen Diabetikern signifikant höhere enddiastolische Füllungsdrücke als bei nicht-insulinpflichtigen gefunden wurden. Diese anhand von neueren echokardiographischen Methoden gewonnen Erkenntnisse weisen darauf hin, dass eine diastolische Funktion sich mit einer intensivierten Diabetes-Therapie als indirekter Parameter für die Schwere der Erkrankung verschlechtert. Es bleibt jedoch zu berücksichtigen, dass sich in der hier untersuchten Kohorte bei weiteren Parametern der diastolischen Funktion wie LAD und LVMI keine Unterschiede zwischen NIDDM und IDDM nachweisen ließen. Zukünftige Studien werden zeigen, ob durch neuere diagnostische Mittel die hier gezeigten Hinweise auf Unterschiede zwischen insulinpflichtigen und nicht-insulinpflichtigen Diabetikern bestätigt werden können.

\subsection{Therapeutische Ansätze}

Eine diastolische Dysfunktion birgt die Gefahr des Übergangs in eine symptomatische diastolische Herzinsuffizienz. Aus den oben dargestellten Ergebnissen dieser Studie lässt sich ableiten, dass beim Diabetiker im asymptomatischen Stadium der Erkrankung zumindest eine adäquate Blutzuckereinstellung zur Prävention einer diastolischen Herzinsuffizienz beitragen kann. Ob schon im asymptomatischen Stadium einer diastolischen Dysfunktion gezielt medikamentös therapiert werden sollte, ist bislang nicht erforscht. Aber auch die Therapie einer diastolischen Herzinsuffizienz ist weitaus weniger klar definiert als die der systolischen Herzinsuffizienz. Neben der symptomatischen und der nichtmedikamentösen Therapie werden bezüglich der medikamentösen Therapie verschiedenste Ansätze diskutiert. Primär zielen diese auf eine Normalisierung des Blutdrucks, Minderung der linksventrikulären Hypertrophie, Verhindern einer Tachykardie, Reduktion des pulmonalvenösen Druckes und Aufrechterhalten der atrialen Kontraktion ab (Hunt et al. 2001; Zile und Brutsaert 2002b). Diuretika und 
Vasodilatatoren reduzieren linksventrikuläre diastolische Drücke. Um eine linksventrikuläre Hypertrophie bedingt durch eine Aktivierung des RAAS-Systems zu mindern, wird eine Therapie mit Aldosteron-Antagonisten empfohlen (Weber und Brilla 1991; Mottram et al. 2004). Einige kleinere klinische Studien zeigten, dass eine Therapie mit $\beta$-Blockern, Calcium-Kanal-Blockern oder AT1-Antagonisten die körperliche Belastungsfähigkeit von Patienten mit diastolischer Herzinsuffizienz erhöhen kann (Bonow et al. 1985; Warner et al. 1999).

In der im Rahmen dieser Arbeit untersuchten Kohorte wurden Diabetiker im Vergleich zu Nicht-Diabetikern häufiger mit ACE-Hemmern, AT1-Rezeptor-Antagonisten, Betablockern, Diuretika, Aldosteron- und Calcium-Antagonisten, sowie Statinen, Aminosalicylaten (ASA) und Vitamin-K-Antagonisten therapiert. Obwohl der Nutzen einer medikamentösen Therapie im asymptomatischen Stadium einer diastolischen Dysfunktion noch nicht belegt und die intensivere Therapie sicherlich mit durch ausgeprägtere kardiale Symptome und häufigere kardiovaskuläre Begleiterkrankungen der Diabetiker bedingt ist, wäre es denkbar, dass diese intensivere Therapie für die Diabetiker auch durch eine positive Beeinflussung der diastolischen Funktion von Nutzen ist.

\subsection{Geschlechtsspezifische Unterschiede}

Wachter et al. zeigten ebenfalls im Rahmen der KNHI-Studie, dass sich bei Hypertonikern ein negativer Einfluss des Diabetes auf die diastolische Funktion primär auf männliche Patienten beschränkte (Wachter et al. 2007). In unseren Analysen fanden sich bei Frauen höhere E/e'-Werte, jedoch keine Unterschiede bezüglich der $\mathrm{HbA1c}$-Werte. Frauen leiden insgesamt häufiger an einer diastolischen Dysfunktion oder einer diastolischen Herzinsuffizienz (Ahmed et al. 2002; Masoudi et al. 2003). Diese Ergebnisse legen insgesamt die Vermutung nahe, dass die diastolische Ventrikelfunktion bei Frauen zwar weniger durch einen Diabetes, jedoch umso stärker durch andere Faktoren verschlechtert wird.

Verschiedene Studien untermauern die Annahme, dass geschlechtsspezifische Unterschiede bezüglich der Reaktion des Herzens auf chronische Belastungen und Beeinträchtigungen existieren. Eine Studie an Patienten mit isoliertem systolischem Hypertonus zeigte bei Frauen eine stärkere konzentrische linksventrikuläre Hypertrophie im Echokardiogramm (Krumholz et al. 1993). Diese Form ist bei einer 
diastolischen Dysfunktion im Gegensatz zur exzentrischen Hypertrophie bei systolischer Dysfunktion häufiger (Schmidt und Pieske 2005). Außerdem bewirkte der Hypertonus bei Frauen eine Zunahme der linksventrikulären Wanddicke ohne eine Dilatation, während bei Männern eine linksventrikuläre Dilatation zu finden war. Diese Ergebnisse legen nahe, dass ein Hypertonus bei Frauen durch eine konzentrische Hypertrophie und Wanddickenzunahme in stärkerem Maße die diastolische Funktion des Ventrikels beeinträchtigt, bei Männern hingegen durch eine Dilatation die systolische Funktion verschlechtert wird. Shigematsu et al. fanden außerdem, dass ein Übergewicht bei Frauen einen stärkeren Einfluss auf die linksventrikuläre Masse hatte als bei Männern (Shigematsu et al. 2006). Eine Zunahme der linksventrikulären Masse geht wiederum mit einer Verschlechterung der diastolischen Funktion einher (Esper et al. 1993).

Den hier dargestellten Erkenntnissen einer schlechteren diastolischen Funktion bei Frauen trotz gleicher $\mathrm{HbA1C}$-Werte widersprechen allerdings Ergebnisse der Framingham-Studie. Rutter et al. fanden, übereinstimmend mit unseren Ergebnissen, einen Anstieg der linksventrikulären Masse und der linksventrikulären Wanddicke mit einer Verschlechterung der Glucoseintoleranz. In der Framingham-Studie war dieser Effekt jedoch bei Frauen ausgeprägter (Rutter et al. 2003). Auch eine Follow-upStudie an 5491 hospitalisierten Patienten mit Herzinsuffizienz ergab, dass ein Diabetes mellitus das Mortalitätsrisiko bei Frauen stärker erhöhte als bei Männern (Gustafsson et al. 2004). Obwohl einige frühere Studien ebenfalls zu dem Ergebnis kommen, dass sich ein Diabetes auf die Prognose einer Herzinsuffizienz bei Frauen schlechter auswirkt (Savage et al. 1988; Ho et al. 1993), muss beachtet werden, dass bei den genannten Studien nicht die diastolische Herzinsuffizienz im Speziellen betrachtet wurde. Denkbar wäre zum Beispiel, dass ein Diabetes mellitus bei Frauen eher zu einer Verschlechterung der systolischen Herzinsuffizienz und in der Folge zu erhöhten Mortalitätsraten führt, während bei Männern die Mortalitätsrate über eine Verschlechterung der diastolischen Funktion beeinflusst wird.

Eine genauere Erforschung der Unterschiede bezüglich des Einflusses eines Diabetes auf das Herz bei Männern und Frauen wird sicher Gegenstand zukünftiger Forschung sein. 


\subsection{6-Minuten-Gehtest}

Die körperliche Leistungsfähigkeit, gemessen anhand des 6-Minuten-Gehtests, verschlechterte sich signifikant mit dem Grad der Glucosestoffwechsel-Störung. Dies ist sicher zum Teil dadurch bedingt, dass Diabetiker häufiger Komorbiditäten aufweisen, wie auch im hier untersuchten Kollektiv (vgl. Tab. 6). Koronare Herzkrankheit und Herzinsuffizienz waren bei den Diabetikern signifikant häufiger vorhanden und dürften die körperliche Leistungsfähigkeit eingeschränkt haben. Einen wesentlichen Einfluss hatte vermutlich auch das Vorhandensein einer peripheren arteriellen Verschlusskrankheit (pAVK), bei der sich die Gehstrecke im 6Minuten-Gehtest abhängig vom Schweregrad signifikant verkürzt (Montgomery et al. 1998). Die pAVK ist eine häufige Komplikation einer Diabeteserkrankung. Mit der Dauer der Diabeteserkrankung erhöht sich das Risiko eine pAVK zu entwickeln (AlDelaimy et al. 2004), daher ist es nicht verwunderlich, dass in der hier untersuchten Kohorte die Prävalenz einer pAVK mit dem Grad der Glucosestoffwechsel-Störung, die ja auch mit der Erkrankungsdauer in Zusammenhang steht (vgl. Tab. 5), anstieg. Bei der Gruppe der insulinpflichtigen Diabetiker war eine PAVK fünf mal häufiger als bei Patienten mit normaler Glucosehomöostase.

Zusätzlich zu diesen Einflüssen der Komorbiditäten auf die körperliche Leistungsfähigkeit, zeigte sich aber auch eine hochsignifikant negative Korrelation zwischen E/e' und Gehstrecke. Dies bestätigt Ergebnisse von Poirier et al., die zeigten, dass die maximale Leistungsfähigkeit auf einem Laufband bei Typ-2Diabetikern durch die diastolische Dysfunktion beeinflusst wurde (Poirier et al. 2000). Auch eine neueren Studie fand eine Assoziation von verringerter körperlicher Leistungsfähigkeit und diastolischer Dysfunktion bei Patienten mit Diabetes mellitus, obwohl deren Blutzucker gut eingestellt war und sie weder an einer koronaren Herzerkrankung, noch an einem Hypertonus oder einer Herzinsuffizienz litten (Saraiva et al. 2005).

Die erläuterten Ergebnisse verdeutlichen, dass das Sinken der Leistungsfähigkeit mit dem Grad einer Glucosestoffwechsel-Störung zum einen durch eine erhöhte Prävalenz von Komorbiditäten, zum anderen durch die Verschlechterung einer diastolischen Dysfunktion bedingt ist.

Eine weitere Schlussfolgerung aus diesen Ergebnissen ist, dass eine diastolische Dysfunktion im asymptomatischen Stadium zwar vom Patienten nicht explizit bemerkt 
wird, sich aber dennoch in einer Verschlechterung der physischen Leistungsfähigkeit äußert und somit die Lebensqualität beeinträchtigen kann.

\subsection{Limitationen}

In der vorliegenden Untersuchung wurde mit 1085 Patienten ein relativ großes Patientenkollektiv untersucht. Dieses setzte sich jedoch aus einer kardiovaskulären Risikogruppe zusammen, sodass kein direkter Rückschluss auf die Allgemeinbevölkerung möglich war. Bei Studien, die Mechanismen der diabetischen Kardiomyopathie untersuchen, werden Patienten mit kardiovaskulären Erkrankungen wie koronarer Herzerkrankung oder chronischer Herzinsuffizienz häufig von den Analysen ausgeschlossen (Poirier et al. 2001; Diamant et al. 2003). Auf eine solche Einschränkung wurde hier bewusst verzichtet, um den Zusammenhang zwischen Glucosemetabolismus und diastolischer Funktion im Ganzen zu erfassen, ungeachtet eventueller Einflüsse von Endorganerkrankungen. Die Tatsache, dass Glucosemetabolismus und diastolische Funktion signifikant assoziiert blieben, wenn KHK und chronische Herzinsuffizienz als Kovariaten bei den Analysen berücksichtigt wurden, weist aber auf einen unabhängigen Einfluss des Glucosemetabolismus hin. Einen weiteren limitierenden Faktor stellt die Tatsache dar, dass hier Daten einer Querschnittsstudie analysiert wurden. Die Daten wurden zu einem einzigen Zeitpunkt erhoben und in gewissen Abständen wiederholt, um Trends in der Entwicklung zu untersuchen. Kausale Zusammenhänge können mit diesem Studiendesign jedoch nicht überprüft werden, da keine Angaben zur zeitlichen Abfolge von Exposition und Outcome (in diesem Fall Diabetes mellitus und diastolische Funktion) zur Verfügung stehen. Die Querschnittsstudie ist somit primär ein Mittel zur Generierung, nicht aber zur Überprüfung von Hypothesen (Gullberg 1996; Touzet und Colin 1999). Die Ergebnisse dieser Studie können folglich nur Hinweise auf einen Einfluss des Diabetes auf die diastolische Dysfunktion liefern.

Der Referenzbereich des hier verwendeten HbA1c-Assays war geringer als bei den meisten üblichen Assays (vgl. zum Beispiel Diamant et al. 2003). Dies schränkt zwar die Vergleichbarkeit mit anderen Untersuchungen ein, hat jedoch keinen Einfluss auf die Validität der Ergebnisse innerhalb unserer Studie.

Als Goldstandard für die Diagnose einer diastolischen Dysfunktion gilt noch immer die Untersuchung mittels Konduktanzkathetersystem (Little et al. 1990; Zile et al. 
2004). Diese Untersuchung ist jedoch zum einen eine invasive Methode, birgt also dementsprechende Risiken für den Patienten, zum anderen ist sie wesentlich aufwendiger. Bei einem Kollektiv von über 1000 Patienten ist eine solche Methode somit weder ethisch noch finanziell vertretbar. Hinzu kommt, dass die Echokardiographie nicht zuletzt durch immer höhere Sensitivität wie den Gewebedoppler und die praktikablere Anwendung an Akzeptanz gewinnt.

Für die Einteilung einer diastolischen Funktion in die verschiedenen Schweregrade existiert kein einheitliches Diagnoseschema. Pritchett et al. verwendeten beispielsweise 2005 ein, dem hier verwendeten zwar im Grundsatz ähnliches Diagnoseschema, Unterschiede bestehen jedoch zum einen bezüglich der Grenzwerte der E/A- und E/e'-Ratio, zum anderen ziehen Pritchett et al. zusätzlich die E-Wellen-Dezelerationszeit (EDCT) zur Schweregradeinteilung heran. Auch Wachter et al. publizierten 2007 ein ähnliches Schema wie das hier verwendete, allerdings bestimmen auch bei diesem zum Teil andere Parameter wie EDCT, IVRT und e' die Schweregradeinteilung. Ein Schema, bei welchem ähnliche Grenzwerte für E/A-, E/e'- und S/D-Ratio gelten, stellte Galderisi 2005 vor, zusätzlich werden hier isovolumetrische Realaxationszeit, atrialer Rückfluss und Dezelerationszeit in die Einteilung einbezogen.

Zusammenfassend ergeben sich also Limitierungen dieser Arbeit im Wesentlichen durch die Selektion einer kardiovaskulären Risikogruppe, das Design einer Querschnittsstudie, den vergleichsweise geringeren Referenzbereich des HbA1cAssays, sowie durch ein fehlendes einheitliches Diagnoseschema für die Schweregrade einer diastolischen Dysfunktion. 


\section{Zusammenfassung}

Diabetes mellitus ist ein unabhängiger Risikofaktor für kardiovaskuläre Erkrankungen. Unabhängig von koronarer Herzerkrankung oder Bluthochdruck tritt eine Schädigung des Herzens auf, die als diabetische Kardiomyopathie bezeichnet wird. Diese äußert sich primär in einer diastolischen Funktionsstörung mit verminderter Relaxation des Ventrikels. Erhöhte linksventrikuläre Füllungsdrücke und ein vermindertes Schlagvolumen sind die Folge. Im Stadium einer diastolischen Dysfunktion ist der Patient noch asymptomatisch, echokardiographisch können jedoch schon Zeichen einer Funktionsstörung gefunden werden. Diabetiker weisen im Vergleich zu Nicht-Diabetikern eine wesentlich höhere Prävalenz der diastolischen Dysfunktion auf. Insbesondere im Hinblick auf mögliche präventive Maßnahmen ist daher der Zusammenhang von Glucosehomöostase und diastolischer Funktion von besonderem Interesse.

Um das Verhältnis von Glucosehomöostase und diastolischer Dysfunktion näher zu untersuchen, wurde im Rahmen der prospektiven KNHI-Studie ein Patientenkollektiv von 1085 Patienten im Alter von 50 bis 85 Jahren mit Risikofaktoren für eine diastolische Herzinsuffizienz oder mit einer manifesten Herzinsuffizienz untersucht. In einem Zeitraum von drei Jahren wurde jährlich eine Untersuchung durchgeführt, die unter anderem eine echokardiographische Untersuchung, Laboranalysen und einen 6-Minuten-Gehtest beinhaltete. Das Kollektiv wurde nach dem Grad der Glucosehomöostase in die Gruppen ,Normal', ,NIDDM' (nicht-insulinpflichtiger Diabetes mellitus) und ,IDDM' (insulinpflichtiger Diabetes mellitus) unterteilt.

Das mittlere Alter der Patienten betrug 66 Jahre. Frauen und Männer waren im Kollektiv etwa gleich stark repräsentiert. Eine diastolische Dysfunktion bestand bei 76\% der Nicht-Diabetiker. Diabetiker wiesen verglichen mit Nicht-Diabetikern eine signifikant höhere Prävalenz einer diastolischen Dysfunktion auf, am häufigsten waren nicht-insulinpflichtige Diabetiker betroffen (86\%). Außerdem waren Diabetiker nicht nur häufiger, sondern auch schwerer von einer diastolischen Dysfunktion betroffen. Die systolische Ventrikelfunktion der Diabetiker hingegen unterschied sich nicht von der der Kontrollgruppe. Bei Betrachtung einzelner echokardiographischer Parameter der diastolischen Funktion zeigte sich, dass Diabetiker sowohl bezüglich der E/e'-Ratio, als Indikator des linksventrikulären enddiastolischen Drucks, als auch bezüglich linksatrialen Durchmessers (LAD) und linksventrikulären Massenindexes 
(LVMI) signifikant schlechtere Werte aufwiesen als Nicht-Diabetiker. Zudem konnte für alle drei Parameter eine Korrelation mit Parametern des Glucosestoffwechsels gefunden werden.

Insgesamt zeigten unsere Untersuchungen, dass eine Assoziation von Glucosemetabolismus und diastolischer Funktion besteht. Die Ergebnisse legen den Schluss nahe, dass die Entstehung oder zumindest das Fortschreiten einer diastolischen Funktionsstörung beim Diabetiker durch gute Blutzuckereinstellung verhindert, beziehungsweise abgeschwächt werden könnte. 


\section{Abkürzungsverzeichnis}

A

$A^{\prime}$

Abb.

ACE

ADA

AGE

AT1

ASA

ASE

ATS

AUC

AUC Glu0-120

AUC normiert

BMI

$\mathrm{CHF}$

$\mathrm{cm}$

cw Doppler

DD

DIAST

dl

E

$e^{\prime}$

EDCT

EDV

EF

eGFR

EKG

ESC

FPG

FPI

FS

g
Einstromgeschwindigkeit nach der Vorhofkontraktion

spätdiastolische Mitralanulusgeschwindigkeit

Abbildung

Angiotensin-converting-Enzym

American Diabetes Association

Advanced glycosylation end products

Angiotensin-II-Rezeptor Typ 1

Aminosalicylate

American Society of Echocardiology

American Thoracic Society

Fläche unter der Kurve

Fläche unter der Kurve im Zeitintervall 0 bis 120 Minuten

normierte Fläche unter der Kurve

Body mass index

chronische Herzinsuffizienz

Zentimeter

kontinuierlicher Doppler

diastolische Dysfunktion

diastolische

Deziliter

frühdiastolische Einstromgeschwindigkeit

frühdiastolische Mitralanulusgeschwindigkeit

E-Wellen-Dezelerationszeit

enddiastolisches Volumen

Ejektionsfraktion

geschätzte Glomeruläre Filtrationsrate

Echokardographie

European Society of Cardiology

Nüchternglucose im Plasma

Nüchterninsulin im Plasma

prozentuale Durchmesserverkürzung

Gramm 
h

HbA1c

HDL

HLA

HOMA-IR

ICD

IDDM

IE

IFG

IVRT

IVS

IVSD

$\mathrm{kg}$

KHK

$\mathrm{KNHI}$

KOF

I

LAD

LADA

LDL

$\log$

LVD

LVEDD

LVEDV

LVEF

LVESV

LVM

LVMI

LVPW

LVV

M

m

$\mathrm{m}^{2}$
Stunde

Hämoglobin A1c: an Glucose gebundenes Hämoglobin

Lipoprotein höherer Dichte

Humanes Leukozyten Antigen

Homöostase-Modell zur Beurteilung der Insulin Resistenz

International Statistical Classification of Diseases and Related

Health Problems

Insulinpflichtiger Diabetes mellitus

Internationale Einheiten

abnorme Nüchternglucose

isovolumetrische Relaxationszeit

enddiastolische interventrikuläre Septumdicke

enddiastolischer Durchmesser des Ventrikelseptums

Kilogramm

koronare Herzkrankheit

Kompetenznetzwerk Herzinsuffizienz

Körperoberfläche

Liter

linksatrialer endsystolischer Durchmesser

Später Autoimmuner Diabetes bei Erwachsenen

Lipoprotein niederer Dichte

Logarithmus

endsystolischer Durchmesser

enddiastolischer Ventrikeldurchmesser

linksventrikuläres enddiastolisches Volumen

linksventrikuläre Ejektionsfraktion

linksventrikuläres endsystolisches Volumen

linksventrikuläre Masse

linksventrikulärer Massenindex

enddiastolische Hinterwanddicke

enddiastolische Füllungsvolumina

Median der Stichprobe

Meter

Quadratmeter 


$\begin{array}{ll}\text { Max. } & \text { Maximum } \\ \text { Min. } & \text { Minimum } \\ \text { min } & \text { Minute } \\ \text { ml } & \text { Milliliter } \\ \text { mm } & \text { Millimeter } \\ \text { mmHg } & \text { Millimeter Quecksilbersäule } \\ \text { mmol } & \text { Millimol } \\ \text { MODY } & \text { Maturity onset diabetes of the young } \\ \text { MRT } & \text { Magnetresonanztomographie } \\ \text { ms } & \text { Millisekunden } \\ \text { H } & \text { Mikro } \\ \text { NIDDM } & \text { nicht-insulinpflichtiger Diabetes mellitus } \\ \text { n.s. } & \text { nicht signifikant } \\ \text { oGTT } & \text { oraler Glucosetoleranztest } \\ \text { pAVK } & \text { periphere arterielle Verschlusskrankheit } \\ \text { PVAF } & \text { atrialer Rückfluss in der Pulmonalvene } \\ \text { PVDF } & \text { diastolischer Pulmonalvenenfluss } \\ \text { PVSF } & \text { systolischer Pulmonalvenenfluss } \\ \text { PWD } & \text { enddiastolischer Durchmesser der Hinterwand } \\ \text { pW-Doppler } & \text { gepulster Doppler } \\ \text { QUICKI } & \text { Quantitativer Insulin-Sensitivitäts-Index } \\ \text { RAAS } & \text { Renin-Angiotensin-Aldosteron-System } \\ \text { RR } & \text { Blutdruck } \\ \text { RR syst. } & \text { systolischer Blutdruck } \\ \text { RR diast. } & \text { diastolischer Blutdruck } \\ \text { S } & \text { Sekunde } \\ \text { S' } & \text { anterograde systolische Welle } \\ \text { s.o. } & \text { siehe oben } \\ \text { Tab. } & \text { Tabelle } \\ \text { TDI } & \text { Gewebedopplerechokardiographie } \\ \text { THV } & \\ \text { U } & \text { Taille-Hüfte-Verhältnis } \\ \text { vgl. } & \text { WHO Health Organization } \\ & \end{array}$




\section{Literaturverzeichnis}

Abhayaratna WP, Marwick TH, Smith WT, Becker NG (2006): Characteristics of left ventricular diastolic dysfunction in the community: an echocardiographic survey. Heart $\underline{92}, 1259-1264$

Ahmed A, Roseman JM, Duxbury AS, Allman RM, DeLong JF (2002): Correlates and outcomes of preserved left ventricular systolic function among older adults hospitalized with heart failure. Am Heart J 144, 365-372

Al-Delaimy WK, Merchant AT, Rimm EB, Willett WC, Stampfer MJ, Hu FB (2004): Effect of type 2 diabetes and its duration on the risk of peripheral arterial disease among men. Am J Med 116, 236-240

Allison DB, Zhu SK, Plankey M, Faith MS, Heo M (2002): Differential associations of body mass index and adiposity with all-cause mortality among men in the first and second National Health and Nutrition Examination Surveys (NHANES I and NHANES II) follow-up studies. Int J Obes Relat Metab Disord 26, 410-416

American Diabetes Association (1998): Economic consequences of diabetes mellitus in the U.S. in 1997. Diabetes Care 21, 296-309

American Diabetes Association (2009): Diagnosis and Classification of Diabetes Mellitus. Diabetes Care $\underline{32}, 62-67$

American Thoracic Society (2002): Guidelines for the Six-Minute Walk Test - ATS Statement. Am J Respir Crit Care Med 166, 111-117

Anderson EA, Hoffman RP, Balon TW, Sinkey CA, Mark AL (1991): Hyperinsulinemia produces both sympathetic neural activation and vasodilation in normal humans. $J$ Clin Invest $\underline{87}, 2246-2252$

Appleton CP, Hatle LK, Popp RL (1988): Demonstration of restrictive ventricular physiology by Doppler echocardiography. J Am Coll Cardiol 11, 757-768 
Apstein CS, Morgan JP: Cellular mechanisms underlying left ventricular diastolic dysfunction; in: Left Ventricular Diastolic Dysfunction and Heart Failure; hrsg. v. Gaasch WH, LeWinter MM; Lea \& Febiger, Philadelphia 1994, 3-24

Aretaios, aus dem Griechischen von Mann A: Die auf uns gekommenen Schriften des Kappadocier Aretaeus. hrsg. v. Sändig M; C.E.M Pfeffer, Halle 1858, Unveränderter Neudruck 1969

Armitage, $P$ (1955): Tests for linear trends in proportions and frequencies. Biometrics $\underline{11}, 375-386$

Assmann G, Schulte H (1988): The Prospective Cardiovascular Münster (PROCAM) study: prevalence of hyperlipidemia in persons with hypertension and/or diabetes mellitus and the relationship to coronary heart disease. Am Heart J 116, 1713-1724

Astorri E, Fiorina P, Contini GA, Albertini D, Magnati G, Astorri A, Lanfredini M (1989): Isolated and preclinical impairment of left ventricular filling in insulindependent and non-insulin-dependent diabetic patients. Clin Cardiol 20, 536-540

Astorri E, Fiorina P, Gavaruzzi G, Astorri A, Magnati G (1997): Left ventricular function in insulin-dependent and in non-insulin-dependent diabetic patients: radionuclide assessment. Cardiology $\underline{88}, 152-155$

Atkinson MA, Maclaren NK (1994): The pathogenesis of insulin dependent diabetes. N Engl J Med $\underline{331}, 1428-1436$

Aurigemma GP, Gottdiener JS, Shemanski L, Gardin J, Kitzman D (2001): Predictive value of systolic and diastolic function for incident congestive heart failure in the elderly: The Cardiovascular Health Study. J Am Coll Cardiol 37, 1042-1048

Barnett AH, Eff C, Leslie RD, Pyke DA (1981): Diabetes in identical twins: A study of 200 pairs. Diabetologia $\underline{20}$ (2), 87-93 
Bell DS (1995): Diabetic cardiomyopathy. A unique entity or a complication of coronary artery disease? Diabetes Care $\underline{18}, 708-14$

Bella JN, Palmieri V, Roman MJ, Liu JE, Welty TK, Lee ET, Fabsitz RR, Howard BV, Devereux RB (2002): Mitral Ratio of Peak Early to Late Diastolic Filling Velocity as a Predictor of Mortality in Middle-Aged and Elderly Adults: The Strong Heart Study. Circulation $\underline{105}$, 1928-1933

Benjamin EJ, Levy D, Vaziri SM, D'Agostino RB, Belanger AJ, Wolf PA (1994): Independent risk factors for atrial fibrillation in a population-based cohort. The Framingham Heart Study. JAMA $\underline{271}$, 840-844

Bewick V, Cheek L, Ball J (2004): Statistics review 10: Further nonparametric methods. Crit Care $\underline{8}, 196-199$

Bogardus C, Lillioja S, Mott DM, Hollenbeck C, Reaven G (1985): Relationship between degree of obesity and in vivo insulin action in man. Am J Physiol 248, 286291

Bonow RO, Dilsizian V, Rosing DR, Maron BJ, Bacharach SL, Green MV (1985): Verapamil-induced improvement in left ventricular diastolic filling and increased exercise tolerance in patients with hypertrophic cardiomyopathy: short- and long-term effects. Circulation $\underline{72}, 853-864$

Borgia MC, Pellicelli AM, Medici F, Barbo Cosial J, Cabañero J, Huelmos A, De Paola G, Lionetti M (1999): Left ventricular filling in young patients affected by insulindependent diabetes mellitus: a stress Doppler echocardiography study. Minerva Endocrinol 24, 97-102

Boyer JK, Thanigaraj S, Schechtman KB, Perez JE (2004): Prevalence of ventricular diastolic dysfunction in asymptomatic, normotensive patients with diabetes mellitus. Am J Cardiol $\underline{93}$ (7), 870-875 
Brilla CG, Maisch B, Weber KT (1992): Myocardial collagen matrix remodelling in arterial hypertension. Eur Heart J $\underline{13}, 24-32$

Brutsaert DL, Fransen P, Andries LJ, De Keulenaer GW, Sys SU (1998): Cardiac endothelium and myocardial function. Cardiovasc Res $\underline{38}, 281-290$

Candido R, Forbes JM, Thomas MC, Vicki Thallas V, Dean RG, Burns WC, Tikellis C, Ritchie RH, Twigg SM, Cooper ME, Burrell LM (2003): A Breaker of Advanced Glycation End Products Attenuates Diabetes-Induced Myocardial Structural Changes. Circ Res $\underline{92}, 785-792$

Castel MA, Méndez F, Tamborero D, Mont L, Magnani S, Tolosana JM, Berruezo A, Godoy M, Sitges M, Vidal B, Roig E, Brugada J (2009): Six-minute walking test predicts long-term cardiac death in patients who received cardiac resynchronization therapy. Europace 11, $338-342$

Celentano A, Vaccaro O, Tammaro P, Galderisi M, Crivaro M, Oliviero M, Imperatore G, Palmieri V, lovino V, Riccardi G, de Divitis O (1995): Early abnormalities of cardiac function in non-insulin-dependent diabetes mellitus and impaired glucose tolerance. Am J Cardiol $\underline{76}$, 1173-1176

Cheitlin MD, Armstrong WF, Aurigemma GP, Beller GA, Bierman FZ, Davis JL, Douglas PS, Faxon DP, Gillam LD, Kimball TR, Kussmaul WG, Pearlman AS, Philbrick JT, Rakowski H, Thys DM, Antman EM, Smith SC, Alpert JS, Gregoratos G, Anderson JL, Hiratzka LF, FAxon DP, Hunt SA, Fuster V, Jacobs AK, Gibbons RJ, Russell RO (2003): Guideline update for the clinical application of echocardiography: a report of the American College of Cardiology/American Heart Association Task force on Practice Guidelines (ACC/AHA/ASE Committee to Update the 1997 Guidelines for the Clinical Application of Echocardiography). Circulation 108, 1146 1162

Cochran WG (1954): Some methods of strengthening the common $\times 2$ tests. Biometrics $\underline{10}$, 417-451 
Cohen GI, MD, Pietbolungo JF, Thomas JD, Klein AL (1998): A Practical Guide to Assessment of Ventricular Diastolic Function Using Doppler Echocardiography. J Am Coll Cardiol 27, 1753-1760

Corcoran CD, Mehta CR (2002): Exact Level and Power of Permutation, Bootstrap and Asymptotic Tests of Trend. J Mod Appl Stat Methods 1, 42-51

Devereux RB, Alonso DR, Luta EM, Gottlieb GJ, Campo E, Sachs I (1986): Echocardiographic assessment of left ventricular hypertrophy: comparison to necropsy findings. Am J Cardiol $\underline{57}$, 450-458

Diamant M, Lamb HJ, Groeneveld Y, Endert EL, Smit JW, Bax JJ, Romijn JA, de Roos A, Radder JK (2003): Diastolic dysfunction is associated with altered myocardial metabolism in asymptomatic normotensive patients with well-controlled type 2 diabetes mellitus. J Am Coll Cardiol $\underline{42}$, 328-335

Di Bonito P, Cuomo S, Moio N, Sibilio G, Sabatini D, Quattrin S, Capaldo B (1996): Diastolic dysfunction in patients with non-insulin-dependent diabetes mellitus of short duration. Diabet Med 13, 321-324

Dickstein K, Cohen-Solal A, Filippatos G, McMurray JJV, Ponikowski P, Poole-Wilson PA, Strömberg A, van Veldhuisen DJ, Atar D, Hoes AW, Keren A, Mebazaa A, Nieminen M, Priori SG, Swedberg K (2008): ESC Guidelines for the diagnosis and treatment of acute and chronic heart failure. Eur Heart J $\underline{29}$, 2388-2442

Erbel R, Neumann T, Zeidan Z, Bartel T, Buck T (2002): Echokardiographische Diagnostik der diastolischen Herzinsuffizienz. Herz $\underline{27}$, 99-106

Esper RJ, Burrieza OH, Cacharrón JL, Fábregues G, Baglivo HP (1993): Left ventricular mass regression and diastolic function improvement in mild and moderate hypertensive patients treated with lisinopril. Cardiology $\underline{83}, 76-81$

European Study Group on Diastolic Heart Failure (1998): How to diagnose diastolic heart failure. Eur Heart J $\underline{19}$ (7), 990-1003 
Expert Committee on the Diagnosis and Classification of Diabetes Mellitus (2003): Report of the Expert Committee on the Diagnosis and Classification of Diabetes Mellitus. Diabetes Care $\underline{26}, 5-20$

Factor SM, Minase T, Sonnenblick EH (1980): Clinical and morphological features of human hypertensive-diabetic cardiomyopathy. Am Heart J $\underline{99}$, 446-458

Factor SM, Bhan R, Minase T, Wolinsky H, Sonnenblick EH (1981): Hypertensivediabetic cardiomyopathy in the rat: an experimental model of human disease. Am J Pathol $\underline{102}, 219-228$

Fang ZY, Prins JB, Marwick TH (2004): Diabetic Cardiomyopathy: Evidence, Mechanisms, and Therapeutic Implications. Endocr Rev $\underline{25}$, 543-567

Feinberg WM, Blackshear JL, Laupacis A, Kronmal R, Hart RG (1995): Prevalence, age distribution, and gender of patients with atrial fibrillation. Analysis and implications. Arch Intern Med 155, 469-473

Fischer M, Baessler A, Hense HW, Hengstenberg C, Muscholl M, Holmer S, Döring A, Broeckel U, Riegger G, Schunkert H (2003): Prevalence of left ventricular diastolic dysfunction in the community. Results from a Doppler echocardiographic-based survey of a population sample. Eur Heart J $\underline{24}, 320-328$

From AM, Scott CG, Chen HH (2009): Changes in Diastolic Dysfunction in Diabetes Mellitus Over Time. Am J Cardiol 103, 1463-1466

From AM, Scott CG, Chen HH (2010): The development of heart failure in patients with diabetes mellitus and pre-clinical diastolic dysfunction a population-based study. J Am Coll Cardiol $\underline{55}$, 300-305

Fujimoto WY, Leonetti DL, Kinyoun JL, Shuman WP, Stolov WC, Wahl PW (1987): Prevalence of complications among second-generation Japanese-American men with diabetes, impaired glucose tolerance or normal glucose tolerance. Diabetes $\underline{36}, 730$ 739 
Fukui S, Fukumoto Y, Suzuki J, Saji K, Nawata J, Shinozaki T, Kagaya Y, Watanabe J, Shimokawa H (2009): Diabetes mellitus accelerates left ventricular diastolic dysfunction through activation of the renin-angiotensin system in hypertensive rats. Hypertens Res $\underline{32}, 472-480$

Fung JW, Li TS, Choy DK, Yip GW, Ko FW, Sanderson JE, Hui DS (2002): Severe obstructive sleep apnea is associated with left ventricular diastolic dysfunction. Chest 121, 422-429

Galderisi M (2005): Diastolic dysfunction and diastolic heart failure: diagnostic, prognostic and therapeutic aspects. Cardiovasc Ultrasound $\underline{3}, 9-23$

Galderisi M, Anderson KM, Wilson PW, Levy D (1991): Echocardiographic evidence for the existence of a distinct diabetic cardiomyopathy (the Framingham Heart Study). Am J Cardiol $\underline{68}, 85-89$

Gelber RP, Gaziano JM, Orav EJ, Manson JE, Buring JE, Kurth T (2008): Measures of obesity and cardiovascular risk among men and women. J Am Coll Cardiol $\underline{52}$, 605-615

Giere W: ICD-10, Diagnosenthesaurus, 1. Auflage; Urban \& Fischer Verlag, München 2000

Gough SC, Smyllie J, Barker M, Berkin KE, Rice PJ, Grant PJ (1995): Diastolic dysfunction is not related to changes in glycaemic control over 6 months in type 2 (non-insulin-dependent) diabetes mellitus. A cross-sectional study. Acta Diabetol $\underline{32}$, 110-115

Grandi AM, Piantanida E, Franzetti I, Bernasconi M, Maresca A, Marnini P, Guasti L, Venco A (2006): Effect of glycemic control on left ventricular diastolic function in type 1 diabetes mellitus. Am J Cardiol $\underline{97}, 71-76$ 
Grausenburger P (2006): Echokardiographie aktuell: Hämodynamische Evaluierung beim Intensivpatienten. J Kardiol $\underline{13}$, 221-224

Grossman E, Shemesh J, Shamiss A, Thaler M, Carroll J, Rosenthal T (1992): Left ventricular mass in diabetes-hypertension. Arch Intern Med 152, 1001-1004

Gullberg B (1996): Design and statistical problems in prevention. Scand J Rheumatol $\underline{103}, 108-110$

Gustafsson I, Brendorp B, Seibaek M, Burchardt H, Hildebrandt P, Køber L, TorpPedersen C; Danish Investigatord of Arrhythmia and Mortality on Dofetilde Study Group (2004): Influence of diabetes and diabetes-gender interaction on the risk of death in patients hospitalized with congestive heart failure. J Am Coll Cardiol $\underline{43}, 771$ 777

Gutierrez C, Blanchard DG (2004): Diastolic heart failure: Challenges of diagnosis and treatment. Am Fam Physician $\underline{69}$, 2609-2616

Guyatt GH, Sullivan MJ, Thompson PJ, Fallen EL, Pugsley SO, Taylor DW, Berman LB (1985): The 6-minute walk: a new measure of exercise capacity in patients with chronic heart failure. Can Med Assoc J 132 (8), 919-923

Gwathmey JK, Warren SE, Briggs GM, Copelas L, Feldman MD, Phillips PJ, Callahan M, Schoen FJ, Grossman W, Morgan J P (1991): Diastolic dysfunction in hypertrophic cardiomyopathy: Effect on active force generation during systole. J Clin Invest $\underline{87}, 1023-1031$

Haffner SM, Lehto S, Rönnemaa T, Pyörälä K, Laakso M (1998): Mortality from coronary heart disease in subjects with type 2 diabetes and in nondiabetic subjects with and without prior myocardial infarction. N Engl J Med 339, 229-234

Henschen F (1969): On the term diabetes in the works of Aretaeus and Galen. Med Hist $\underline{13}$, 190-192 
Hense HW (2000): Epidemiologie der arteriellen Hypertonie und Implikationen für die Prävention. 10-Jahres-Ergebnisse der MONICA-Studie Augsburg. Dtsch Med Wochenschr $\underline{125}, 1397-1402$

Hess OM (2003): Herzinsuffizienz: Definition, Ursachen und Formen. Schweiz Med Forum $\underline{48}, 1158-1163$

Ho KK, Anderson KM, Kannel WB, Grossman W, Levy D (1993): Survival after the onset of congestive heart failure in Framingham Heart Study subjects. Circulation $\underline{88}$, 107-115

Holmäng A, Yoshida N, Jennische E, Waldenström A, Björntorp P (1996): The effects of hyperinsulinaemia on myocardial mass, blood pressure regulation and central haemodynamics in rats. Eur J Clin Invest $\underline{26}$, 973-978

Hunt SA, Baker DW, Chin MH, Cinquegrani MP, Feldman AM, Francis GS, Ganiats TG, Goldstein S, Gregoratos G, Jessup ML, Noble RJ, Packer M, Silver MA, Stevenson LW, Gibbons RJ, Antman EM, Alpert JS, Faxon DP, Fuster V, Jacobs AK, Hiratzka LF, Russell RO, Smith SC Jr; American College of Cardiology/American Heart Association (2001): ACC/AHA guidelines for the evaluation and management of chronic heart failure in the adult: executive summary. A report of the American College of Cardiology/American Heart Association Task Force on Practice Guidelines (Committee to revise the 1995 Guidelines for the Evaluation and Management of Heart Failure). J Am Coll Cardiol $\underline{38}$, 2101-2113

Ingelsson E, Sundström J, Arnlöv J, Zethelius B, Lind L (2005): Insulin resistance and risk of congestive heart failure. JAMA 294, 334-341

International Expert Committee (2009): International Expert Committee report on the role of the $\mathrm{A} 1 \mathrm{C}$ assay in the diagnosis of diabetes. Diabetes Care $\underline{32}, 1327-1334$

Iribarren C, Karter AJ, Go AS, Ferrara A, Liu JY, Sidney S, Selby JV (2001): Glycemic control and heart failure among adult patients with diabetes. Circulation $\underline{103}, 2668-2773$ 
Jarnert C, Landstedt-Hallin L, Malmberg K, Melcher A, Ohrvik J, Persson H, Rydén L (2009): A randomized trial of the impact of strict glycaemic control on myocardial diastolic function and perfusion reserve: a report from the DADD (Diabetes mellitus And Diastolic Dysfunction) study. Eur J Heart Fail 11, 39-47

Jonckheere AR (1954): A distribution-free k-sample test against ordered alternatives. Biometrica $\underline{41}, 133-45$

Kannel WB, Hjortland M, Castelli WP (1974): Role of diabetes in congestive heart failure: the Framingham study. Am J Cardiol 34, 29-34

Kawaguchi M, Techigawara M, Ishihata T, Asakura T, Saito F, Maehara K, Maruyama $Y$ (1997): A comparison of ultrastructural changes on endomyocardial biopsy specimens obtained from patients with diabetes mellitus with and without hypertension. Heart Vessels $\underline{12}, 267-274$

Kerner W, Brückel J: Definition, Klassifikation und Diagnostik des Diabetes mellitus; in: Praxis-Leitlinien der Deutschen Diabetes-Gesellschaft (DDG). Diabetologie und Stoffwechsel; hrsg. v. Scherbaum WA; Band 3, Supplement 2; Georg Thieme Verlag KG, Stuttgart 2008, S. 131-133

Kerner W, Brückel J: Definition, Klassifikation und Diagnostik des Diabetes mellitus; in: Diabetologie und Stoffwechsel - Supplement 2 Praxis Leitlinien der Deutschen Diabetes Gesellschaft; hrsg. v. Kellerer M, Danne T; Georg Thieme Verlag Stuttgart 2009, 115-117

Khavandi K, Khavandi A, Asghar O, Greenstein A, Withers S, Heagerty AM, Malik RA (2009): Diabetic cardiomyopathy - a distinct disease? Best Pract Res Clin Endocrinol Metab 23, 347-360 
Kitzman DW, Sheikh KH, Beere PA, Philips JL, Higginbotham MB (1991): Agerelated alterations of Doppler left ventricular filling indexes in normal subjects are independent of left ventricular mass, heart rate, contractility and loading conditions. J Am Coll Cardiol 18, 1243-1250

Kitzman DW, Gardin JM, Gottdiener JS, Arnold A, Boineau R, Aurigemma G, Marino EK, Lyles M, Cushman M, Enright PL; Cardiovascular Health Study Research Group (2001): Importance of heart failure with preserved systolic function in patients $>$ or $=$ 65 years of age. CHS Research Group. Cardiovascular Health Study. Am J Cardiol $\underline{87}, 413-419$

Kizer JR, Bella JN, Palmieri V, Liu JE, Best LG, Lee ET, Roman MJ, Devereux RB (2006): Left atrial diameter as an independent predictor of first clinical cardiovascular events in middle-aged and elderly adults: the Strong Heart Study (SHS). Am Heart J $\underline{151}, 412-418$

Krumholz HM, Larson M, Levy D (1993): Sex differences in cardiac adaptation to isolated systolic hypertension. Am J Cardiol $\underline{72}, 310-313$

Kuecherer HF, Muhiudeen IA, Kusumoto FM, Lee E, Moulinier LE, Cahalan MK, Schiller NB (1990): Estimation of mean left atrial pressure from transesophageal pulsed Doppler echocardiography of pulmonary venous flow. Circulation $\underline{82}$ (4), 1127-1139

Kuznetsova T, Herbots L, López B, Jin Y, Richart T, Thijs L, González A, Herregods MC, Fagard RH, Díez J, Staessen JA (2009): Prevalence of left ventricular diastolic dysfunction in a general population. Circ Heart Fail $\underline{2}$ (2), 105-112

Lam CS, Borlaug BA, Kane GC, Enders FT, Rodeheffer RJ, Redfield MM (2009): Age-associated increases in pulmonary artery systolic pressure in the general population. Circulation 119, 2663-2270 
Levy D, Garrison RJ, Savage DD, Kannel WB, Castelli WP (1990): Prognostic implications of echocardiographically determined left ventricular mass in the Framingham Heart Study. N Engl J Med $\underline{322}$, 1561-1566

Liebl A, Neiß A, Spannheimer A, Reitberger U, Wagner T, Görtz A (2001): Kosten des Typ-2-Diabetes in Deutschland: Ergebnisse der CODE-2®-Studie. Dtsch Med Wochenschr $\underline{126}$, 585-589

Lindström J, Tuomilehto J (2003): The Diabetes Risk Score: A practical tool to predict type 2 diabetes risk. Diabetes Care $\underline{26}, 725-731$

Lippi G, Mattiuzzi C, Targher G (2010): Glycated hemoglobin, diabetes, and cardiovascular risk in nondiabetic adults. N Engl J Med 362, 2030

Little WC, Downes TR, Applegate RJ (1990): Invasive evolution of left ventricular diastolic performance. Herz $\underline{15}, 362-376$

Loimaala A, Groundstroem K, Rinne M, Nenonen A, Huhtala H, Vuori I (2007): Exercise training does not improve myocardial diastolic tissue velocities in Type 2 diabetes. Cardiovasc Ultrasound $\underline{5}, 32$

Masoudi FA, Havranek EP, Smith G, Fish RH, Steiner JF, Ordin DL, Krumholz HM (2003): Gender, age, and heart failure with preserved left ventricular systolic function. J Am Coll Cardiol 41, 217-223

Masugata H, Senda S, Goda F, Yoshihara Y, Yoshikawa K, Fujita N, Daikuhara H, Okuyama H, Taoka T, Kohno M (2008): Left ventricular diastolic dysfunction in normotensive diabetic patients in various age strata. Diabetes Res Clin Pract $\underline{79}, 91-$ 96

McKee PA, Castelli WP, McNamara PM, Kannel WB (1971): The natural history of congestive heart failure: the Framingham study. N Engl J Med 285, 1441-1446 
Medici F, Hawa M, lanari A, Pyke DA, Leslie RDG (1999): Concordance rate for Type II diabetes mellitus in monozygotic twins: actuarial analysis. Diabetologia $\underline{42}, 146-150$

Meigs JB, Nathan DM, D'Agostino RB, Wilson PWF (2002): Fasting and Postchallenge Glycemia and Cardiovascular Disease Risk: The Framingham Offspring Study. Diabetes Care $\underline{25}, 1845-1850$

Miyazato J, Horio T, Takishita S, Kawano Y (2002): Fasting plasma glucose is an independent determinant of left ventricular diastolic dysfunction in nondiabetic patients with treated essential hypertension. Hypertens Res $\underline{25}$ (3), 403-409

Mizushige K, Yao L, Noma T, Kiyomoto H, Yu Y, Hosomi N, Ohmori K, Matsuo H (2000): Alteration in left ventricular diastolic filling and accumulation of myocardial collagen at insulin-resistant prediabetic stage of a type II diabetic rat model. Circulation 101, 899-907

Montgomery PS, Gardner AW (1998): The clinical utility of a six-minute walk test in peripheral arterial occlusive disease patients. J Am Geriatr Soc $\underline{46}, 706-711$

Mottram PM, Haluska B, Leano R, Cowley D, Stowasser M, Marwick TH (2004): Effect of aldosterone antagonism on myocardial dysfunction in hypertensive patients with diastolic heart failure. Circulation $\underline{110}, 558-565$

Movahed MR, Hashemzadeh M, Jamal MM (2005): Diabetes mellitus is a strong, independent risk for atrial fibrillation and flutter in addition to other cardiovascular disease. Int J Cardiol $\underline{105}$, 315-318

Myreng Y, Smiseth OA (1990): Assessment of left ventricular relaxation by Doppler echocardiography. Comparison of isovolumic relaxation time and transmitral flow velocities with time constant of isovolumic relaxation. Circulation $\mathbf{8 1}, 260-266$

Nagueh SF, Middelton KJ, Kopelen HA, Zoghbi WA, Quinones MA (1997): Doppler tissue imaging: a non-invasive technique for evaluation of left ventricular relaxation and estimation of filling pressures. J Am Coll Cardiol $\underline{30}$, 1527-1533 
Nichols GA, Reinier K, Chugh SS (2009): Independent contribution of diabetes to increased prevalence and incidence of atrial fibrillation. Diabetes Care $\underline{32}, 1851-1856$

Nishimura RA, Tajik AJ (1997): Evaluation of Diastolic Filling of Left Ventricle in Health and Disease: Doppler Echocardiography Is the Clinician's Rosetta Stone. J Am Coll Cardiol 30, 8-18

Ohno M, Cheng CP, Little WC (1994): Mechanism of altered patterns of left ventricular filling during the development of congestive heart failure. Circulation $\underline{89}$, 2241-2250

Ohno T, Horio F, Tanaka S, Terada M, Namikawa T, Kitoh J (2000): Fatty liver and hyperlipidemia in IDDM (insulin-dependent diabetes mellitus) of streptozotocintreated shrews. Life Sci $\underline{66}, 125-131$

O'Keeffe ST, Lye M, Donnellan C, Carmichael DN (1998): Reproducibility and responsiveness of quality of life assessment and six minute walk test in elderly heart failure patients. Heart $\underline{80}, 377-382$

Olefsky JM, Kolterman OG, Scarlett, JA (1982): Insulin action and resistance in obesity and noninsulin-dependent type II diabetes mellitus. Am J Physiol Endocrinol Metab $\underline{243}, 15-30$

Ommen SR, Nishimura RA (2003): A clinical approach to the assessment of left ventricular diastolic function by Doppler echocardiography: update 2003. Heart $\underline{89}$, $18-23$

Ommen SR, Nishimura RA, Appleton CP, Miller FA, Oh JK, Redfield MM, Tajik AJ (2000): Clinical Utility of Doppler Echocardiography and Tissue Doppler Imaging in the Estimation of Left Ventricular Filling Pressures: A Comparative Simultaneous Doppler-Catheterization Study. Circulation 102, 1788-1794 
Orchard TJ, Dorman JS, Maser RE, Becker DJ, Drash AL, Ellis D, LaPorte RE, Kuller LH (1990): Prevalence of complications in IDDM by sex and duration. Pittsburgh Epidemiology of Diabetes Complications Study II. Diabetes $\underline{39}, 1116-1124$

Owan TE, Hodge DO, Herges RM, Jacobsen SJ, Roger VL, Redfield MM (2006): Trends in prevalence and outcome of heart failure with preserved ejection fraction. $\mathrm{N}$ Engl J Med $\underline{355}$, 251-259

Palmieri V, Bella JN, Arnett DK, Liu JE, Oberman A, Schuck MY, Kitzman DW, Hopkins PN, Morgan D, Rao DC, Devereux RB (2001): Effect of type 2 diabetes mellitus on left ventricular geometry and systolic function in hypertensive subjects: Hypertension Genetic Epidemiology Network (HyperGEN) study. Circulation 103, 102-107

Poirier P, Garneau C, Bogaty P, Nadeau A, Marois L, Brochu C, Gingras C, Fortin C, Jobin J, Dumesnil JG (2000): Impact of left ventricular diastolic dysfunction on maximal treadmill performance in normotensive subjects with well-controlled type 2 diabetes mellitus. Am J Cardiol $\underline{85}, 473-477$

Poirier P, Bogaty P, Garneu C, Marois L, Dumesnil JG (2001): Diastolic Dysfunction in Normotensive Men With Well-Controlled Type 2 Diabetes: Importance of manoeuvres in echocardiographic screening for preclinical diabetic cardiomyopathy. Diabetes Care 24, 5-10

Poirier P, Giles TD, Bray GA, Hong Y, Stern JS, Pi-Sunyer FX, Eckel RH (2006): Obesity and cardiovascular disease: pathophysiology, evaluation, and effect of weight loss: an update of the 1997 American Heart Association Scientific Statement on Obesity and Heart Disease from the Obesity Committee of the Council on Nutrition, Physical Activity and Metabolism. Circulation 113, 898-918

Poornima IG, Parikh P, Shannon RP (2006): Diabetic cardiomyopathy: the search for a unifying hypothesis. Circ Res $\underline{98}, 596-605$ 
Pritchett AM, Mahoney DW, Jacobsen SJ, Rodeheffer RJ, Karon BL, Redfield MM (2005): Diastolic dysfunction and left atrial volume: a population-based study. J Am Coll Cardiol $\underline{45}, 87-92$

Quinones MA, Otto CM, Stoddard M, Waggoner A, Zoghbi WA (2002): Doppler Quantification Task Force of the Nomenclature and Standards Committee of the American Society of Echocardiography. Recommendations for quantification of Doppler echocardiography: a report from the Doppler Quantification Task Force of the Nomenclature and Standards Committee of the American Society of Echocardiography. J Am Soc Echocardiogr 15,167-84

Raev DC (1994): Which left ventricular function is impaired earlier in the evolution of diabetic cardiomyopathy? An echocardiographic study of young type I diabetic patients. Diabetes Care $\underline{17}, 633-639$

Rathmann W, Haastert B, Icks A, Löwel H, Meisinger C, Holle R, Giani G (2003): High prevalence of undiagnosed diabetes mellitus in Southern Germany: target populations for efficient screening. The KORA survey 2000. Diabetologia $\underline{46}, 182-189$

Redfield MM, Jacobsen SJ, Burnett JC, Mahoney DW, Bailey KR, Rodeheffer RJ (2003): Burden of Systolic and Diastolic Ventricular Dysfunction in the Community: Appreciating the Scope of the Heart Failure Epidemic. JAMA 289, 194-202

Regan TJ, Lyons MM, Ahmed SS, Levinson GE, Oldewurtel HA, Ahmad MR, Haider B (1977): Evidence of cardiomyopathy in familial diabetes mellitus. J Clin Invest $\underline{60}$, 884-899

Rijzewijk LJ, van der Meer RW, Smit JW, Diamant M, Bax JJ, Hammer S, Romijn JA, de Roos A, Lamb HJ (2008): Myocardial steatosis is an independent predictor of diastolic dysfunction in type 2 diabetes mellitus. J Am Coll Cardiol $\underline{52}, 1793-1799$

Roglic G, Unwin N, Bennett PH, Mathers C, Nag S, Connolly V (2005): The Burden of Mortality Attributable to Diabetes: Realistic estimates for the year 2000. Diabetes Care $\underline{28}, 2130-2135$ 
Rubler S, Dlugash J, Yuceoglu YZ, Kumral T, Branwood AW, Grishman A (1972): New type of cardiomyopathy associated with diabetic glomerulosclerosis. Am J Cardiol $\underline{30}$, 595-602

Rutter MK, Parise H, Benjamin EJ, Levy D, Larson MG, Meigs JB, Nesto RW, Wilson PW, Vasan RS (2003): Impact of glucose intolerance and insulin resistance on cardiac structure and function: sex-related differences in the Framingham Heart Study. Circulation $\underline{107}, 448-454$

Sanders LJ (2002): From Thebes to Toronto and the 21st Century: An Incredible Journey. Diabetes Spectr $\underline{15}, 56-60$

Saraiva RM, Duarte DM, Duarte MP, Martins AF, Poltronieri AV, Ferreira ME, Silva MC, Hohleuwerger R, Ellis A, Rachid MB, Monteiro CF, Kaiser SE (2005): Tissue Doppler imaging identifies asymptomatic normotensive diabetics with diastolic dysfunction and reduced exercise tolerance. Echocardiography 22, 561-570

Savage MP, Krolewski AS, Kenien GG, Lebeis MP, Christlieb AR, Lewis SM (1988): Acute myocardial infarction in diabetes mellitus and significance of congestive heart failure as a prognostic factor. Am J Cardiol $\underline{62}, 665-669$

Saydah SH, Eberhardt MS, Loria CM (2001): Subclinical states of glucose intolerance and risk of death in the U.S. Diabetes Care $\underline{24}, 447-453$

Schannwell CM, Schneppenheim M, Perings S, Plehn G, Strauer BE (2002): Left ventricular diastolic dysfunction as an early manifestation of diabetic cardiomyopathy. Cardiology $\underline{98}, 33-39$

Schienkiewitz A, Schulze MB, Hoffmann K, Kroke A, Boeing H (2006): Body mass index history and risk of type 2 diabetes: results from the European Prospective Investigation into Cancer and Nutrition (EPIC)-Potsdam Study. Am J Clin Nutr $\underline{84}$, 427-433 
Schiller NB, Shah PM, Crawford M, DeMaria A, Devereux R, Feigenbaum H, Gutgesell H, Reichek N, Sahn D, Schnittger I, Silverman NH, Tajik AJ (1989): Recommendations for quantitation of the left ventricle by two-dimensional echocardiography. American Society of Echocardiography Committee on Standards, Subcommittee on Quantitation of Two-Dimensional Echocardiograms. J Am Soc Echocardiogr $\underline{2}$ (5), 358-367

Schmidt AG, Pieske B (2005): Diastolische Herzinsuffizienz - Diagnostik. Dtsch Med Wochenschr $\underline{130}, 1208-1212$

Schwarz P, Gruhl U, Hoffmann R (2007): Prävention des Metabolischen Syndroms und Typ-2-Diabetes; in: Deutsche Diabetes-Union: Gesundheitsbericht Diabetes 2007; hrsg. v. Deutsche Diabetes-Union, Kirchheim und Co. GmbH, Mainz 2007, 1823

Shigematsu Y, Norimatsu S, Ohtsuka T, Okayama H, Higaki J (2006): Sex-related differences in the relations of insulin resistance and obesity to left ventricular hypertrophy in Japanese hypertensive patients. Hypertens Res $\underline{29}$, 499-504

Smith NL, Barzilay JI, Shaffer D, Savage PJ, Heckbert SR, Kuller LH, Kronmal RA, Resnick HE, Psaty BM (2002): Fasting and 2-Hour Postchallenge Serum Glucose Measures and Risk of Incident Cardiovascular Events in the Elderly: The Cardivascular Health Study. Arch Intern Med 162, 209-216

Stratton IM, Adler Al, Andrew H, Neil W, Matthews DR, Manley SE, Cull CA, Hadden D, Turner RC, Holman RR (2000): Association of glycaemia with macrovascular and microvascular complications of type 2 diabetes (UKPDS 35): prospective observational study. Br Med J $\underline{321}, 405-412$

Taylor HA Jr, Coady SA, Levy D, Walker ER, Vasan RS, Liu J, Akylbekova EL, Garrison RJ, Fox C (2010): Relationships of BMI to cardiovascular risk factors differ by ethnicity. Obesity (Silver Spring) 18 (8), 1638-1645 
Terpstra TJ (1952): The asymptotic normality and consistency of Kendall's test against trend, when ties are present in one ranking. Indagationes Mathematicae $\underline{14}$, 327-333

Tortoledo FA, Quinones MA, Fernandez GC, Waggoner AD, Winters Jr WL (1983): Quantification of left ventricular volumes by two-dimensional echocardiography: a simplified and accurate approach. Circulation $\underline{67}, 579-584$

Touzet S, Colin C (1999): Interpretation of epidemiologic studies. Type of study, elements of bias, causality. Rev Prat $\underline{49}, 1797-1804$

Tsang TSM, Gersh BJ, Appleton CP, Tajik JT, Barnes ME, Bailey KR, Oh JK (2002): Left Ventricular Diastolic Dysfunction as a Predictor of the First Diagnosed Nonvalvular Atrial Fibrillation in 840 Elderly Men and Women. J Am Coll Cardiol 40, $1636-1644$

Vanninen E, Mustonen J, Vainio P, Länsimies E, Uusitupa M (1992): Left ventricular function and dimensions in newly diagnosed non-insulin-dependent diabetes mellitus. Am J Cardiol 70, 371-378

Vasan RS, Levy D (2000): Defining Diastolic Heart Failure: A Call for Standardized Diagnostic Criteria. Circulation 101, 2118-2121

Vazquez G, Duval S, Jacobs DR Jr, Silventoinen K (2007): Comparison of body mass index, waist circumference, and waist/hip ratio in predicting incident diabetes: a meta-analysis. Epidemiol Rev $\underline{29}, 115-128$

Vinereanu D, Nicolaides E, Tweddel AC, Mädler CF, Holst B, Boden LE, Cinteza M, Rees AE, Fraser AG (2003): Subclinical left ventricular dysfunction in asymptomatic patients with Type II diabetes mellitus, related to serum lipids and glycated haemoglobin. Clin Sci (Lond) $\underline{105}$, 591-599 
von Bibra H, Hansen A, Dounis V, Bystedt $T$, Malmberg K, Rydén L (2004): Augmented metabolic control improves myocardial diastolic function and perfusion in patients with non-insulin dependent diabetes. Heart $\underline{90}$, 1483-1484

Wachter R, Lüers C, Kleta S, Griebel K, Herrmann-Lingen C, Binder L, Janicke N, Wetzel D, Kochen MM, Pieske B (2007): Impact of diabetes on left ventricular diastolic function in patients with arterial hypertension. Eur J Heart Fail $\underline{9}$, 469-476

Warner JG Jr, Metzger DC, Kitzman DW, Wesley DJ, Little WC (1999): Losartan improves exercise tolerance in patients with diastolic dysfunction and a hypertensive response to exercise. J Am Coll Cardiol 33 1567-1572

Weber KT (1989): Cardiac interstitium in health and disease: the fibrillar collagen network. J Am Coll Cardiol 13, 1637-1652

Weber KT, Brilla CG (1991): Pathological hypertrophy and cardiac interstitium. Fibrosis and renin-angiotensin-aldosterone system. Circulation $\underline{83}$, 1849-1865

Wild S, Roglic G, Green A, Sicree R, King H (2004): Global Prevalence of Diabetes Estimates for the year 2000 and projections for 2030 Diabetes Care 27, 1047-1053

Wilson PWF, D'Agostino RB, Levy D, Belanger AM, Silbershatz H, Kannel WB (1998): Prediction of Coronary Heart Disease Using Risk Factor Categories. Circulation $\underline{97}$, 1837-1847

Wittchen H, Pieper L, Eichler T, Klotsche J: Prävalenz und Versorgung von Diabetes mellitus und Herz-Kreislauf- Erkrankungen: DETECT - eine bundesweite Versorgungsstudie an über 55.000 Hausarztpatienten; in: Prävention und Versorgungsforschung; hrsg. V. Kirch W, Badura B, Pfaff H; Springer Verlag, Heidelberg 2008, 315-328

Wolf PA, Benjamin EJ, Belanger AJ, Kannel WB, Levy D, D'Agostino RB (1996): Secular trends in the prevalence of atrial fibrillation: The Framingham Study. Am Heart J $\underline{131}, 790-795$ 
World Health Organization: Definition, Diagnosis and Classification of Diabetes Mellitus and its Complications: Report of a WHO Consultation. Part 1: Diagnosis and Classification of Diabetes Mellitus. WHO Document Production Services, Geneva 1999

World Health Organization: Definition and Diagnosis of Diabetes mellitus and intermediate hyperglycemia: Report of a WHO/IDF Consultation. WHO Document Production Services, Geneva 2006

Yamada H, Goh PP, Sun JP, Odabashian J, Garcia MJ, Thomas JD, Klein AL (2002): Prevalence of left ventricular diastolic dysfunction by Doppler echocardiography: clinical application of the Canadian consensus guidelines. J Am Soc Echocardiogr $\underline{15}, 1238-1244$

Yamamoto K, Wilson DJ, Canzanello VJ, Redfield MM (2000): Left ventricular diastolic dysfunction in patients with hypertension and preserved systolic function. Mayo Clin Proc $\underline{75}$, 148-155

Zabalgoitia M, Ismaeil MF, Anderson L, Maklady FA (2000): Prevalence of diastolic dysfunction in normotensive, asymptomatic patients with well-controlled type 2 diabetes mellitus. Am J Cardiol 87, 320-323

Zanati SG, Mouraria GG, Matsubara LS, Giannini M, Matsubara BB (2009): Profile of cardiovascular risk factors and mortality in patients with symptomatic peripheral arterial disease. Clinics (Sao Paulo) 64, 323-326

Zile MR (1989): Diastolic dysfunction: Detection, consequences and treatment. Part I: Definition and determinants of diastolic function. Mod Concepts Cardiovasc Dis $\underline{58}$, $67-71$

Zile MR, Brutsaert DL (2002a): New Concepts in Diastolic Dysfunction and Diastolic Heart Failure: Part I: Diagnosis, Prognosis, and Measurements of Diastolic Function. Circulation 105, 1387-1393 
Zile MR, Brutsaert DL (2002b): New Concepts in Diastolic Dysfunction and Diastolic Heart Failure: Part II: Causal Mechanisms and Treatment. Circulation 105, 15031508

Zile MR, Gaasch WH, Carroll JD, Feldman MD, Aurigemma GP, Schaer GL, Ghali JK, Liebson PR (2001): Heart failure with a normal ejection fraction: is measurement of diastolic function necessary to make the diagnosis of diastolic heart failure? Circulation 104, 779-782

Zile MR, Baicu CF, Gaasch WH (2004): Diastolic heart failure-abnormalities in active relaxation and passive stiffness of the left ventricle. N Engl J Med 350, 1953-1959

Zimmet PZ (1992): Kelly West Lecture 1991: Challenges in diabetes epidemiology: from west to the rest. Diabetes Care $\underline{15}, 232-252$ 


\section{Publikationen zur Dissertation}

Originalarbeit:

Stahrenberg R, Edelmann F, Mende M, Kockskämper A, Düngen HD, Scherer $M$, Kochen MM, Binder L, Herrmann-Lingen C, Schönbrunn L, Gelbrich G, Hasenfuß G, Pieske B, Wachter R (2010): Association of glucose metabolism with diastolic function along the diabetic continuum. Diabetologia $\underline{53}$ (7), 1331-1340

Abstracts:

Stahrenberg R, Edelmann F, Mende M, Kockskämper A, Düngen HD, Scherer $M$, Kochen MM, Binder L, Herrmann-Lingen C, Schönbrunn L, Gelbrich G, Hasenfuß G, Pieske B, Wachter R (2010): Association of glucose metabolism with diastolic function along the diabetic continuum. Clin Res Cardiol $\underline{99}$ (1)

Stahrenberg R, Mende M, Edelmann F, Schönbrunn L, Hasenfuß G, Pieske B, Wachter R (2010): Diastolic function along the diabetic spectrum. J Am Coll Cardiol $\underline{55}$ 


\section{Danksagung}

Zunächst möchte ich mich bei meinem Betreuer Herrn Dr. med. R. Wachter für die Unterstützung während der Planung und Durchführung meiner Arbeit bedanken. $\mathrm{Er}$ war mit seinen konstruktiven Vorschlägen eine große Hilfe und hatte immer ein offenes Ohr für Fragen und Probleme. Darüber hinaus vermittelte er mir klinische Kenntnisse und Fähigkeiten im Bereich der Kardiologie, die mir in meinem zukünftigen beruflichen Alltag sicher sehr hilfreich sein werden.

Ich danke Herrn Prof. Dr. med. G. Hasenfuß für die Überlassung des interessanten Themas und die Übernahme des Referates.

Außerdem bedanke ich mich bei allen Mitarbeitern des $\mathrm{KNHI}$, insbesondere bei Frau D. von Grünhagen für die geduldige Einarbeitung und die freundliche Zusammenarbeit. Mein Dank gilt außerdem den an Diast-CHF teilnehmenden Patienten, sowie allen Mitdoktorandinnen und Doktoranden, insbesondere Frau Manuela Knoke, Frau Kathleen Durstewitz und Frau Frederike Polzin für die freundschaftliche und kollegiale Zusammenarbeit.

Mein Dank gilt außerdem Herrn Dr. med. R. Stahrenberg für die geduldige und hilfsbereite Unterstützung in allen Belangen der Statistik. 


\section{Lebenslauf}

Am 3. April 1985 wurde ich, Lisa Christiane Schönbrunn, als Tochter von Frau Dr. med. vet. Katrin Schönbrunn, geb. Budelmann und Herrn Dr. med. Ekkehard Schönbrunn in Frankfurt am Main geboren. Von 1991 bis 1993 besuchte ich die Grundschule Sackenbach in Lohr am Main und von 1993 bis 1995 die Grundschule Giesen. Im Anschluss besuchte ich das Bischöfliche Gymnasium Josephinum in Hildesheim, welches ich im Jahre 2004 mit Erwerb der Allgemeinen Hochschulreife verließ.

Nach einem einmonatigen Aufenthalt in Togo, Westafrika, 2004, einer zweimonatigen Tätigkeit als Altenpflegehelferin und Ableisten meines Pflegepraktikums am Städtischen Krankenhaus Hildesheim im Jahre 2005 begann ich zum Sommersemester 2005 mein Studium der Humanmedizin an der Georg-AugustUniversität Göttingen. Die Famulaturen im Rahmen des Studiums leistete ich in den Fachbereichen Onkologie, Unfallchirurgie, Pathologie, Neurologie und Kardiologie ab.

Im Februar 2010 begann ich das Praktische Jahr, welches ich in den Bereichen Neurologie, Innere Medizin und Chirurgie in Bremen, Oldenburg und Göttingen absolvierte. Im April 2011 werde ich voraussichtlich den Zweiten Abschnitt der Ärztlichen Prüfung durchlaufen.

Die vorgelegte Dissertation begann ich im Jahre 2008 in der Abteilung Kardiologie und Pneumologie an der Universität Göttingen unter der Doktorvaterschaft von Herrn Prof. Dr. med. G. Hasenfuß und der Betreuung von Herrn Dr. med. Rolf Wachter. 